\title{
Sea container inspection with tagged neutrons
}

\author{
Bertrand Pérot ${ }^{1, *}$, Cédric Carasco ${ }^{1}$, Cyrille Eléon ${ }^{1}$, Sébastien Bernard ${ }^{1}$, Alix Sardet ${ }^{1}$, Wassila El Kanawati ${ }^{1,2}$, \\ Clément Deyglun ${ }^{1,3}$, Grégory Perret ${ }^{1,4}$, Guillaume Sannié ${ }^{5}$, Vladivoj Valkovic ${ }^{6}$, Davorin Sudac ${ }^{6}$, Jasmina Obhodas ${ }^{6}$, \\ Sandra Moretto $^{7}$, Giancarlo Nebbia ${ }^{7}$, Cristiano Fontana ${ }^{7}$, Felix Pino ${ }^{7}$, Antonietta Donzella ${ }^{8,9}$, Aldo Zenoni ${ }^{8,9}$, \\ Alessandro Iovene ${ }^{10}$, Carlo Tintori ${ }^{10}$, Marek Moszynski ${ }^{11}$, and Michal Gierlik ${ }^{11}$ \\ ${ }^{1}$ CEA, DES, IRESNE, CEA Cadarache, F-13108 Saint-Paul-lez-Durance, France \\ 2 Now with Lebanese University, Beirut, Lebanon \\ ${ }^{3}$ Now with IRSN - PDS-DEND/SESN/L2MN, B.P. 17 - 92262 Fontenay-aux-Roses Cedex, France \\ 4 Now with Paul Scherrer Institute, Forschungsstrasse 111, 5232 Villigen PSI, Suisse \\ ${ }^{5}$ CEA, DRT, LIST, Saclay, F-91191 Gif-Sur-Yvette, France \\ ${ }^{6}$ Ruder Boskovic Institute, Bijenickac.54, 10000 Zagreb, Croatia \\ 7 INFN and Università Degli Studi di Padova, via Marzolo, 8, Padova I-35131, Italy \\ 8 Dipartimento di Ingegneria Meccanica e Industriale, Università degli Studi di Brescia, via Branze 38, Brescia 25123, Italy \\ ${ }^{9}$ INFN Sezione di Pavia, Via Bassi, 6, Pavia 27100, Italy \\ 10 CAEN S.p.A., Via Vetraia, 11, Viareggio LU 55049, Italy \\ 11 National Centre for Nuclear Research (NCBJ), Otwock 05-400, Poland
}

Received: 2 December 2020 / Received in final form: 23 January 2021 / Accepted: 10 February 2021

\begin{abstract}
Neutron inspection of sea-going cargo containers has been widely studied in the past 20 yr to nonintrusively detect terrorist threats, like explosives or Special Nuclear Materials (SNM), and illicit goods, like narcotics or smuggling materials. Fast $14 \mathrm{MeV}$ neutrons are produced by a portable generator with the $\mathrm{t}(\mathrm{d}, \mathrm{n}) \alpha$ fusion reaction, and tagged in both direction and time thanks to the alpha particle detection. This Associated Particle Technique (APT) allows focusing inspection on specific areas of interest in the containers, previously identified as containing suspicious items with X-ray radiographic scanners or radiation portal monitors. We describe the principle of APT for non-nuclear material identification, and for nuclear material detection, then we provide illustrations of the performances for $10 \mathrm{~min}$ inspections with significant quantities (kilograms) of explosives, illicit drugs, or SNM, in different cargo cover loads (e.g. metallic, organic, or ceramic matrices).
\end{abstract}

\section{Introduction}

Detecting Chemical, Bacteriological, Radioactive, Nuclear and Explosive (CBRN-E) threats and illicit trafficking (narcotics, smuggling) in commercial cargo containers has become a major security issue since the past $20 \mathrm{yr}$. Currently, radiation portal monitors (RPM) and X-ray scanners are used as 1st-level non-intrusive inspection (NII) techniques in seaports and terrestrial borders. They are able to trigger an alarm in case of radiological threat or illicit trafficking suspicion, in a very short measurement time (from a few seconds up to $1 \mathrm{~min}$ ). If X-ray scanners provide high-resolution two-dimensional density radiographies able to evidence suspicious items, they however give limited information about their elemental composition. This technology is indeed sensitive to the electronic

\footnotetext{
* e-mail: bertrand.perot@cea.fr
}

density, but it is not always able to clarify the nature of the materials. Specific imaging techniques have been developed to enhance the contrast between high-, intermediate- and low-atomic-number elements, such as dual-energy X-ray radiography [1-5], combined fast-neutron and gamma- or $\mathrm{X}$-ray radiography [6], or transmission radiography with spectroscopic detectors [7]. However, despite the constant improvements in imaging techniques, discriminating illicit and benign substances made of light organic elements with similar densities and effective atomic numbers $\left(Z_{\text {eff }}\right)$ remains difficult, especially in the case of cargo materials of different compositions overlapped inside the container. Fast neutron transmission spectroscopy may improve element identification [8-10] but it only provides a projected density of all crossed elements, making interpretation difficult again in case of overlapped materials, and requires that a sufficient neutron flux emerges from the inspected container. In the case of large containers filled with dense organic goods with different compositions, these 
limitations may impair the identification of hidden materials.

Another way to bring information about the elemental composition is to detect gamma rays following neutron interactions in cargo materials [11,12], gamma spectroscopy allows identifying their major elements: hydrogen, carbon, nitrogen, oxygen, chlorine, iron, etc. Discrimination between illicit and benign materials is obtained by the comparison of the relative fractions of the relevant elements. As common goods can be used to mask or "dilute" illegal materials, spatial discrimination is a key issue. Therefore, fast neutron time-offlight measurements have been developed to perform 3D inspections, by using nanosecond pulses [13] or the associated particle technique (APT) [14]. These methods make it possible to localize the volumetric pixel (voxel) in which each gamma ray was produced, which greatly improves the signalto-noise ratio and allows to determine the elemental composition of a suspect item surrounded by benign materials. Fast neutrons are well suited to explore large volume cargo containers because of their high penetration in bulk material, while several-MeV induced gamma rays are capable of escaping cargo materials.

The APT has been known for a long time in the laboratory [15] but its use in field applications was made possible more recently thanks to transportable associated particle neutron generators [16]. The fast $14 \mathrm{MeV}$ neutrons produced in the $\mathrm{D}-\mathrm{T}$ sealed tube are tagged in both direction and time with a position sensitive alpha detector, the alpha particle being emitted almost back-to-back with the neutron, and the alpha-gamma coincidence time giving the neutron time-of-flight (TOF) until its interaction producing the gamma radiation, such as inelastic scattering. The neutron TOF directly gives its flight path, knowing the $5.13 \mathrm{~cm} . \mathrm{ns}^{-1}$ velocity of $14 \mathrm{MeV}$ neutrons. It is thus possible to build the gamma spectrum induced by tagged neutrons in a selected region of interest localized by a $1^{\text {st }}$ level NII X-ray scanner. The main elements present in the inspected voxel are identified by gamma spectroscopy and their proportions are determined taking into account neutron moderation and gamma attenuation in cargo materials $[17,18]$.

The APT can also be used to detect special nuclear materials (SNM) by measuring coincidences between the alpha particle and induced-fission prompt neutrons and gamma rays [19-21]. The detection of SNM has indeed become in recent years a major objective in the fight against terrorist activities. Especially, highly enriched uranium (HEU) is difficult to detect with passive RPM (1st level NII) because of insufficient spontaneous neutron emission and low energy of its most intense gamma or $\mathrm{X}$-rays $(186,144,93.3,163 \mathrm{keV}$, etc.). A dense cargo cover load (such as iron) or a few cm-thick intentional metal shield (such as lead) could stop most of these radiations. Nevertheless, such a dense object might be detected with an X-ray radiographic scanner or residual SNM emissions could trigger an RPM alarm (1st level NII techniques), and therefore, active techniques could be used as 2nd level NII for SNM threat confirmation or cargo container clearance. Many photon and neutron interrogation methods are being investigated for SNM detection [22]. Their principle is to induce fissions in nuclear materials with high-energy photons above the photofission threshold near $6 \mathrm{MeV}$ [7,23-28], or with neutrons ranging from fast to thermal energies [29-34], and to detect induced-fission prompt or delayed particles.

Concerning photofission, the historical method was based on the detection of delayed neutrons [23] but their signal is strongly attenuated in hydrogenous cargo because of their low average energy, which is close to $400 \mathrm{keV}$. Therefore, researches moved quickly towards the detection of photofission delayed gamma rays, especially those with an energy larger than $3 \mathrm{MeV}$ [24] that are less sensitive to attenuation by cargo materials and to interferences with naturally-occurring or activated gamma backgrounds. This technique is today close to a commercial deployment in LINAC-based systems combining X-ray imaging, photofission and other advanced techniques, but it is a costly and fixed installation based on a high power accelerator [28]. On the other hand, one can also mention investigations with nanosecond coincidence analysis between the correlated photofission prompt neutrons and gamma rays, either with a ns-pulsed LINAC [27] or during a continuous low-dose irradiation [26].

Concerning the detection of SNM in cargo containers by neutron interrogation, first investigations used a fast and directional D-D neutron beam produced by a large accelerator and large plastic scintillators to detect induced-fission delayed gamma rays [34]. This large system is however costly and not transportable. Another approach based on a transportable D-T neutron generator and on the well-known differential die-away analysis (DDAA) [35] was then studied to perform thermal neutron interrogation between the pulses of the generator, using detection blocks made of ${ }^{3} \mathrm{He}$ proportional counters embedded in a moderator and wrapped by cadmium sheets [31]. However, besides the shortage risk and high cost of ${ }^{3} \mathrm{He}$ detectors [36], potential limitations occur with the DDAA in case of cargo material containing thermal neutron absorbers (e.g. hydrogen, boron, chlorine, etc.), or when an intentional shield made of high neutron absorbers (lithium, boron enriched in ${ }^{10} \mathrm{~B}$, cadmium, etc.) surrounds the SNM.

In this context, fast neutron inspection with a transportable APT neutron generator and a large array of low-cost plastic scintillators, as presented below, may constitute a valuable complement to photofission in terms of attenuation of interrogating particles in the wide variety of cargo materials. Fast neutrons are indeed more penetrating than photons in dense materials with a high atomic number, like iron and other metals, while high-energy photons are more penetrating in organic and other rich-in-hydrogen materials.

\section{Illicit cargo identification with tagged neutrons}

\subsection{EURITRACK tagged neutron inspection system}

\subsubsection{Overview}

EURITRACK (European Illicit Trafficking Countermeasures Kit) is a project of the 6th European Union Framework 

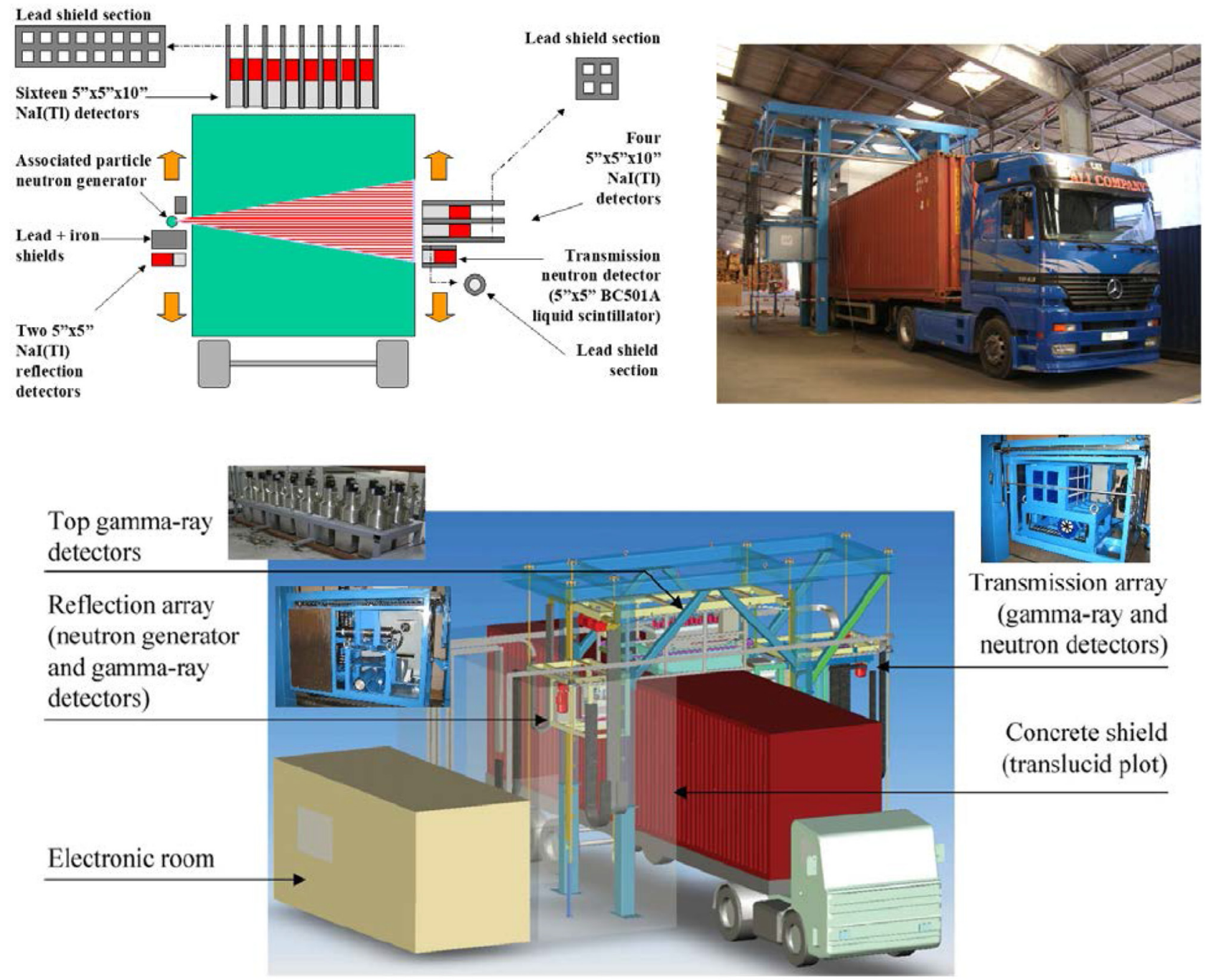

Fig. 1. The EURITRACK Tagged Neutron Inspection System.

Program (FP6), whose aim was to develop a neutron inspection system to detect threat materials (explosives, illicit drugs, etc.) in cargo containers. The Tagged Neutron Inspection System (TNIS) concept has been studied in Europe in the early 2000's for landmine detection [14,16,37]. A truck inspection TNIS has been developed to be used in connection with existing X-ray scanners $[38,39]$. The X-ray radiography (1st level NII) is used to determine the position of a suspicious voxel inside the cargo container that is of interest to be further inspected by the TNIS (2nd level NII). Consequently, it is not necessary to scan the entire container with neutron beams, thus limiting the dose delivered to transported goods. Figure 1 presents the EURITRACK system. The truck stops when the tagged neutron beam is in the area defined on the X-ray radiography, and the neutron generator moves vertically to finely focus the beam on the voxel of interest.

The transportable sealed neutron tube generator includes a $8 \times 8$ matrix of YAP: Ce alpha crystals coupled to a multi-anode photomultiplier [40]. High-efficiency $5^{\prime \prime} \times 5^{\prime \prime}$ and $5^{\prime \prime} \times 5^{\prime \prime} \times 10^{\prime \prime} \mathrm{NaI}(\mathrm{Tl})$ detectors are located around the cargo container to detect neutron-induced gamma rays. The neutron attenuation across the container is measured with a $5^{\prime \prime} \times 5^{\prime \prime} \mathrm{BC}-501 \mathrm{~A}$ liquid scintillation detector, which discriminates neutron and gamma from pulse-shape and time-of-flight information. These detectors are equipped with fast photomultiplier tubes to achieve nanosecond time resolution [41]. A dedicated frontend electronics processes coincidences between any alpha and gamma-ray detectors [42] (Fig. 2).

The design and expected performances of the system have been studied using Monte Carlo simulation with MCNP [43], showing in particular that the EURITRACK TNIS can detect, in $10 \mathrm{~min}$, a $100 \mathrm{~kg}$ block of TNT explosive hidden in a container fully filled with iron freight of $0.2 \mathrm{~g} . \mathrm{cm}^{-3}$ mean density, which was the reference case of the EURITRACK project. This performance has been validated in laboratory conditions $[44,45]$ and the system has been implemented in the seaport of Rijeka, Croatia, to perform a demonstration on real cargo containers [46]. Panel (2) of Figure 3 shows the neutron time-of-flight (converted to flight path) and gamma-ray spectra acquired in $10 \mathrm{~min}$ with a drum containing $75 \mathrm{~kg}$ of 


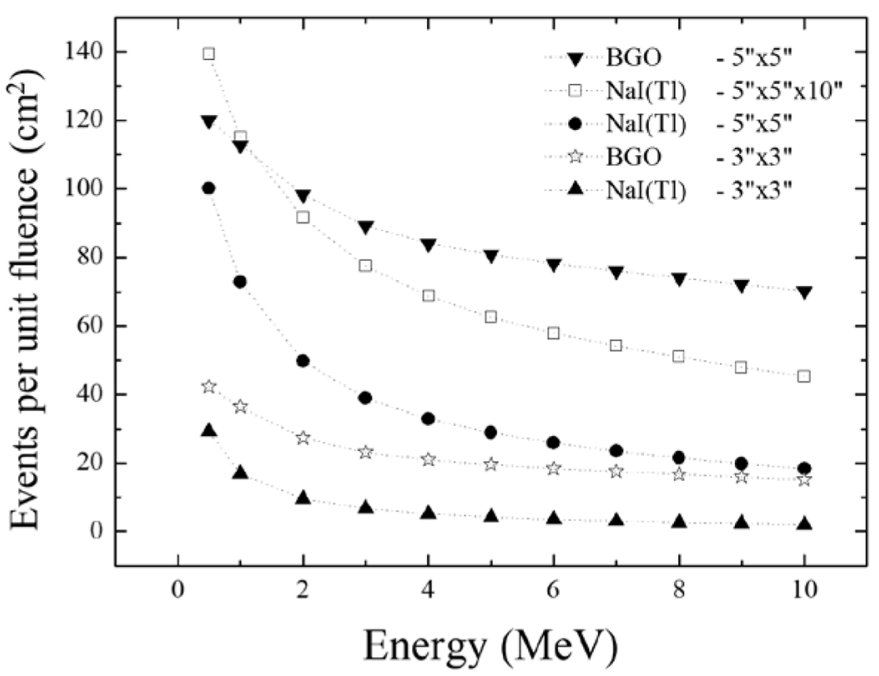

Fig. 2. Sensitivity of large scintillators calculated with MCNP computer code [41]. Sensitivity is defined as the arithmetic product of the full-energy peak intrinsic efficiency (counts per incident photon) and the detector entrance surface. It relates to the probability of detection per unit incident flux (number of detector events for one photon per $\mathrm{cm}^{2}$ reaching the detector). ammonium acetate $\left(\mathrm{C}_{2} \mathrm{O}_{2} \mathrm{NH}_{7}\right)$, which is used as a TNT $\left(\mathrm{C}_{7} \mathrm{H}_{5} \mathrm{O}_{6} \mathrm{~N}_{3}\right)$ surrogate in terms of $\mathrm{C}, \mathrm{N}$, and $\mathrm{O}$ chemical proportions. The drum is placed in the middle of an iron cargo with a 0.2 g. $\mathrm{cm}^{-3}$ average density. Despite the lower density of ammonium acetate powder with respect to of TNT $\left(0.8 \mathrm{~g} . \mathrm{cm}^{-3}\right.$ vs. $\left.1.6 \mathrm{~g} . \mathrm{cm}^{-3}\right)$, its presence is clearly visible in the flight path spectrum, and in turn the gammaray spectrum shows $\mathrm{C}, \mathrm{N}$, and $\mathrm{O}$ peaks that are not present in neighbouring iron areas.

The spectroscopic analysis consists in unfolding the whole gamma-ray spectrum into a linear combination of single elemental spectra (C, N, O, Fe, etc.) with a least square algorithm, see panel (3) of Figure 3. The discrimination between organic materials is a main issue in X-ray radiography because they show similar densities and average atomic numbers; it is here performed with the relative proportions of $\mathrm{C}, \mathrm{N}$, and $\mathrm{O}$ measured with the neutron system, represented in the equilateral triangle of panel (4). In this representation, the distances between the measured point and each side of the triangle correspond to the relative proportions of $\mathrm{C}$ (perpendicular projection on the $\mathrm{O}-\mathrm{N}$ side), $\mathrm{N}$ (projection on $\mathrm{C}-\mathrm{O}$ side) and $\mathrm{O}$ (projection on $\mathrm{C}-\mathrm{N}$ side).

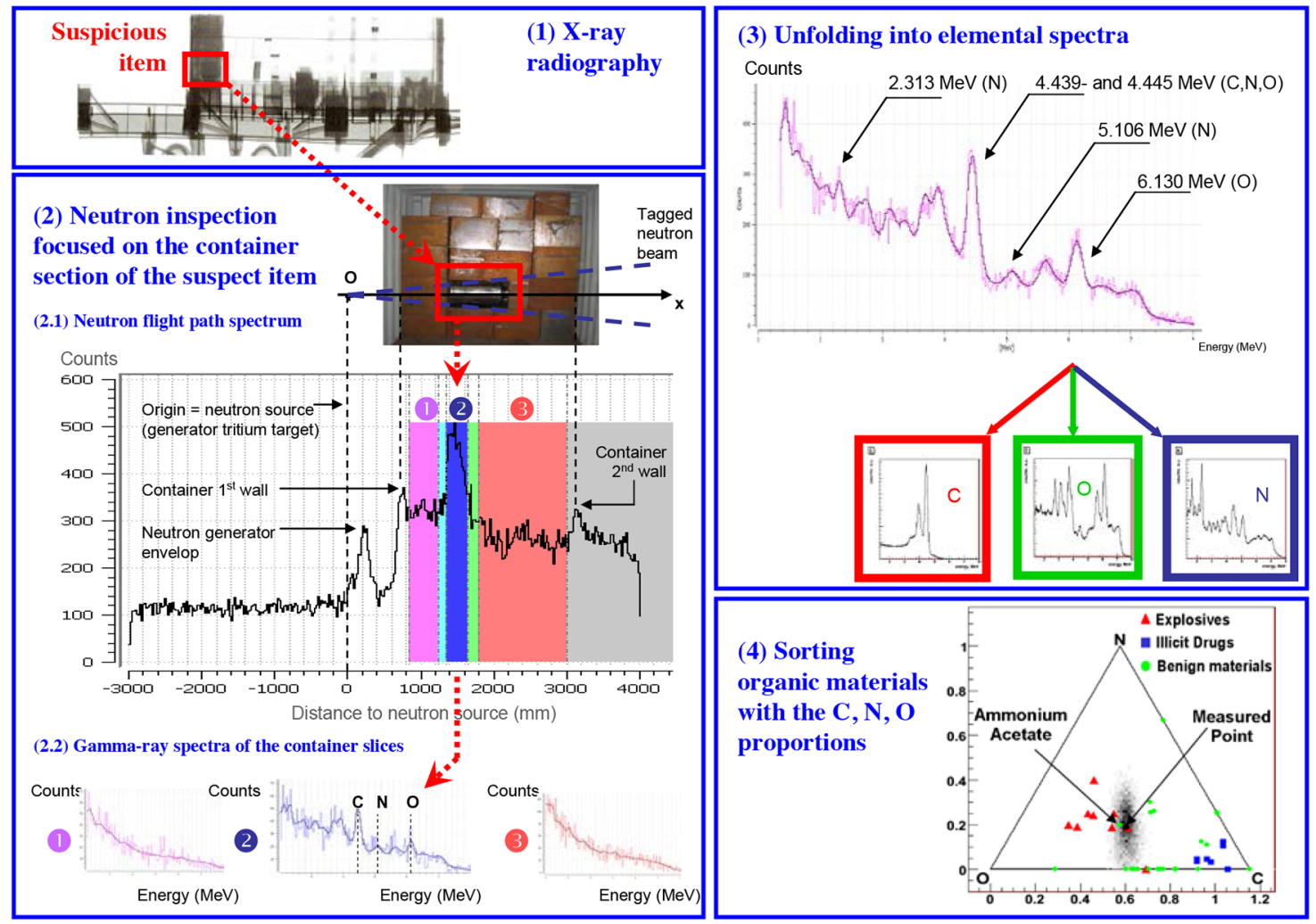

Fig. 3. The EURITRACK inspection concept: (1) X-ray radiography to detect and localise suspicious items inside the cargo container; (2) tagged neutron inspection after focusing the beam on the area of interest; (2.1) neutron flight-path spectrum to determine the depth of interest inside the cargo container; (2.2) gamma-ray spectra corresponding to different "slices" along the tagged neutron beam; (3) gamma spectra unfolding with a linear combination of elemental gamma spectra (C, O, N, Fe...); (4) C, N, and O relative proportions in a triangle representation (see text for details) to sort explosives and narcotics from benign materials. 

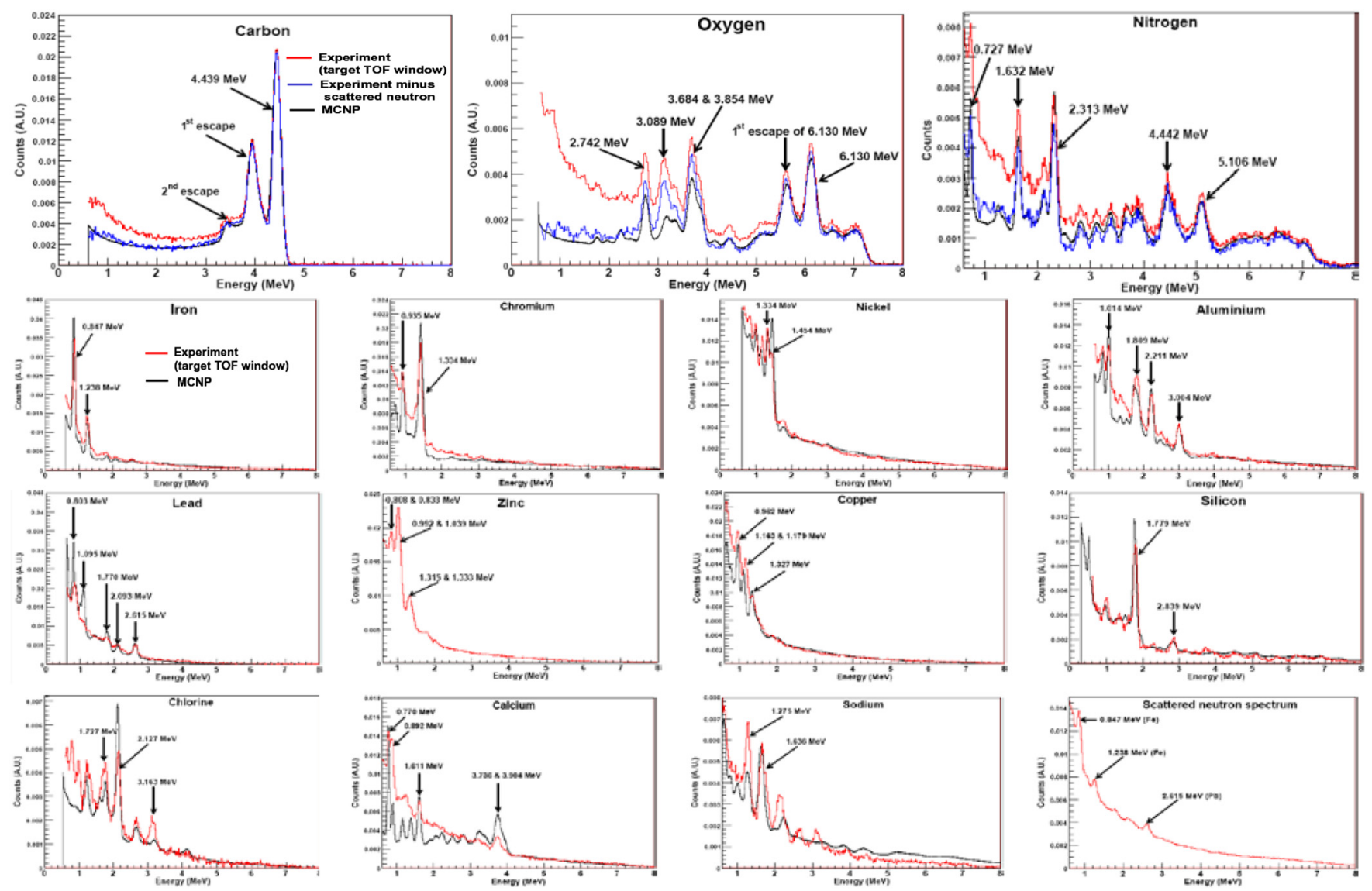

Fig. 4. Pure gamma-ray signatures of elements acquired with the EURITRACK system and comparison with MCNPX [49] simulations performed with the ENDF/B-VII.0 nuclear database. The "scattered neutrons spectrum" corresponds to events recorded just after the sample TOF window as shown in Figure 5.

\subsubsection{Database of elemental gamma-ray spectra}

The calibration of the system requires measurements with reference materials to record the elemental gamma spectra induced by $14 \mathrm{MeV}$ tagged neutrons. Long acquisition times are generally necessary to obtain good counting statistics, especially for nuclei with low fast neutron reaction cross sections leading to the emission of prompt gamma rays [47-49]. For instance, the carbon signature is acquired with graphite, oxygen with water (fast neutrons do not produce gamma ray on hydrogen nuclei), nitrogen with liquid nitrogen in the laboratory and with melamine in the field (seaport of Rijeka). When a mixture like melamine $\left(\mathrm{C}_{3} \mathrm{~N}_{6} \mathrm{H}_{6}\right)$ is used, it is necessary to subtract the carbon signal to obtain the pure nitrogen spectrum.

For target materials with low atomic number elements like hydrogen and carbon, which scatter interrogating neutrons towards the gamma-ray detectors, it is also necessary to subtract the corresponding background, see examples in Figure 4. Scattered neutrons may be directly detected by the $\mathrm{NaI}(\mathrm{Tl})$ crystals or produce gamma rays in the surrounding lead shield and iron parts of the portal. The TOF spectra presented in Figure 5, converted into interaction position spectra, show the overlap between this background and the useful signal. On the other hand, the random background observed in any coincidence technique has also been subtracted; it is here due to uncorrelated neutrons or gamma rays that are accidentally detected during the time window triggered by an alpha particle.

\subsubsection{Experimental validation of MCNP simulations}

The pure element acquisitions have been modelled with the MCNP [50] and MCNPX [51] computer codes to help calibration (peak identification in the gamma-ray spectra) and to validate the simulation method in view of further calculations (in support to data processing). However, discrepancies observed between experiment and calculation for several elements (inconsistent relative pulse heights, absence of peaks, unattended peaks, or even no gamma-ray production data for zinc) have evidenced that a complete numerical calibration of the system is not possible.

In Figure 4, experimental and calculated spectra were compared in a qualitative point of view, after normalization to unity because only the spectrum shape is of interest for the unfolding algorithm. However, in order to classify organic goods in benign materials, explosives, or narcotics (see triangle in Fig. 3), the unfolded $\mathrm{C}, \mathrm{N}$ and $\mathrm{O}$ count fractions are converted into chemical proportions using 

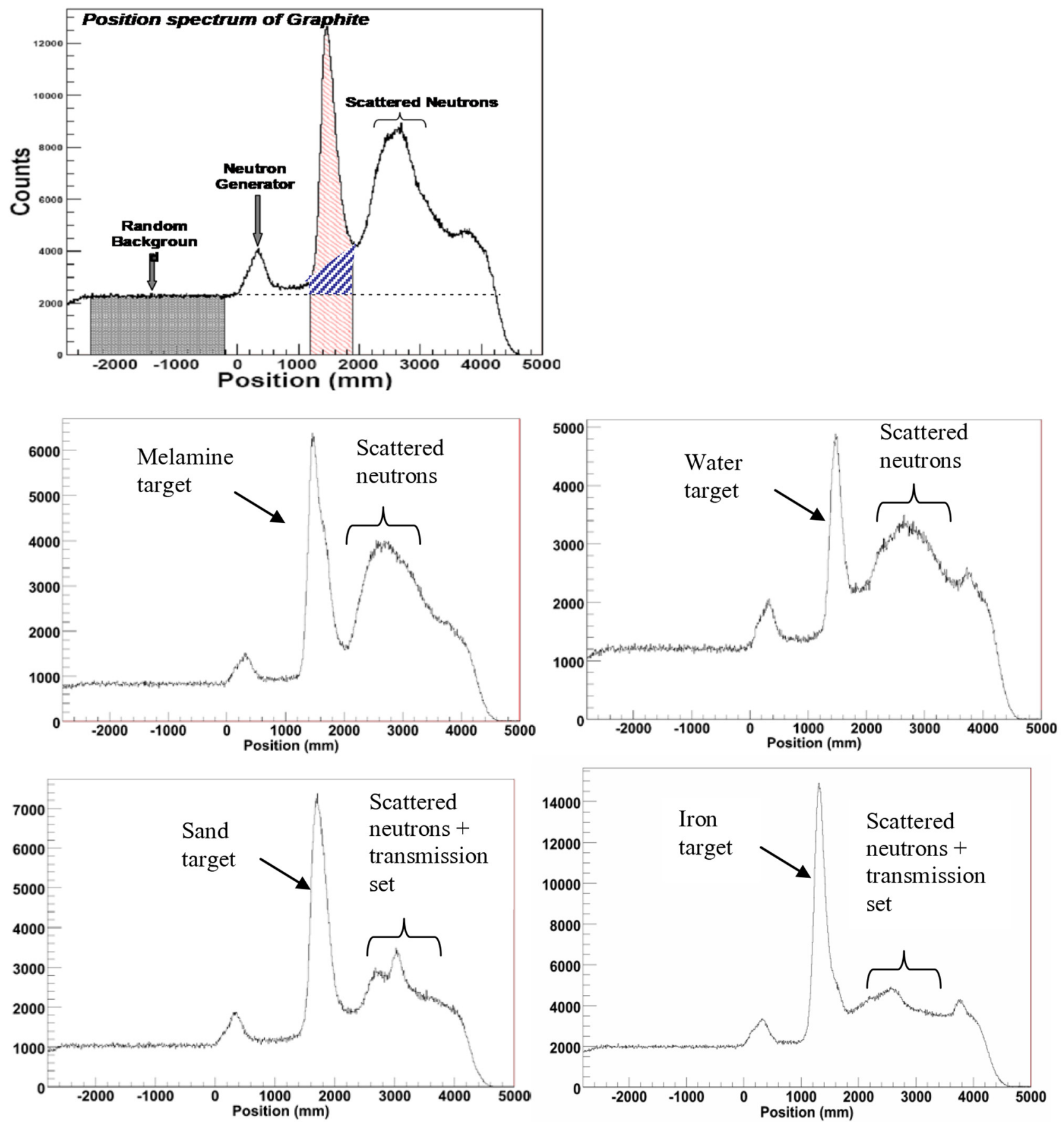

Fig. 5. Distance-to-interaction spectra for different acquisitions with calibration materials. On the first plot showing the graphite spectrum, the blue dashed area corresponds to the fraction of the scattered neutron background overlapping with the carbon signal in the red dashed area. The subtraction result is shown in Figure 4 for $\mathrm{C}, \mathrm{N}$, and O. The random background (grey area) has also been subtracted.

numerical simulation. Therefore, we performed dedicated measurements with a well-known setup to allow a quantitative comparison between experiment and simulation for these three key elements [52]. Figure 6 shows a quite satisfactory agreement, taking into account the uncertainties discussed below. Only the $3.089 \mathrm{MeV}$ gamma ray, due to the relaxation of the first excited level of ${ }^{13} \mathrm{C}$, after the ${ }^{16} \mathrm{O}(\mathrm{n}, \alpha){ }^{13} \mathrm{C}$ reaction, is significantly under- estimated in the oxygen calculated spectrum, as already observed in other calculations with the same ENDF/B-VII.0 library [53]. Fortunately, this discrepancy has a limited impact on material identification, as shown in next sections.

The significant fluctuations observed in these spectra are due to poor counting statistics, as acquisition time was limited in the seaport of Rijeka where these specific 

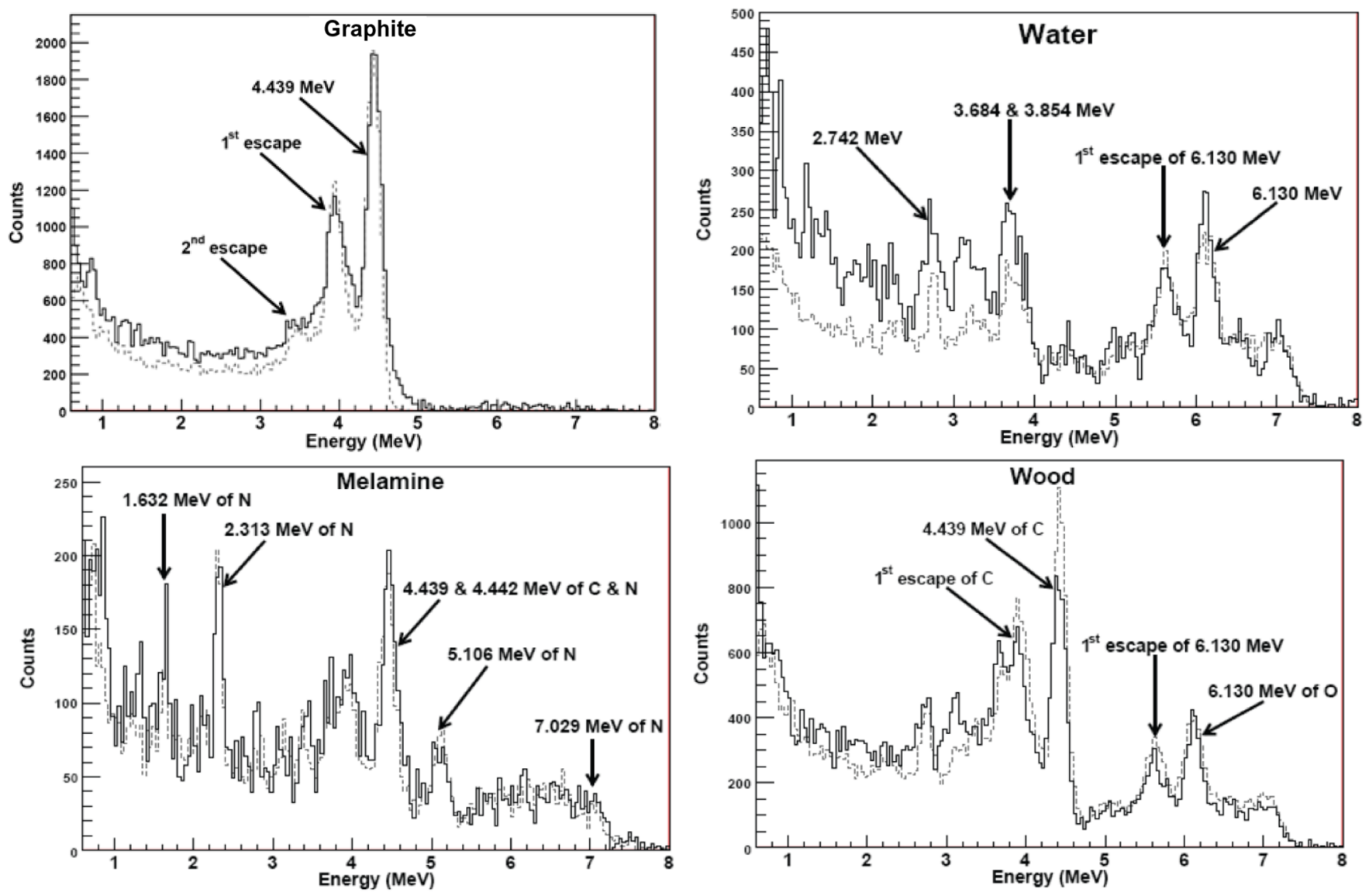

Fig. 6. Quantitative comparison between the experimental (full lines) and calculated (dotted lines) gamma-ray spectra of several target materials.

measurements have been performed. Concerning the potential sources of systematic errors (biases), one can first note that gamma-ray production data by $14 \mathrm{MeV}$ neutrons are reported with large standard deviations, generally in the 10-20\% range and even more for specific gamma rays [54]. The evaluated nuclear data files may also include errors and inconsistencies [55]. Secondly, simulation introduces uncertainties due to the precision of the model (geometry of the setup, material compositions and densities, size and direction of the tagged neutron beam, etc.) or to the specific calculation process, such as the twostep calculation of neutron-induced gamma spectra. Indeed, a time-energy dependant gamma flux reaching each detector is calculated with MCNP "point detector" (F5 tally), followed by a second calculation in which these photons are supposed to enter NaI crystals in normal incidence to calculate the pulse-height spectrum (F8 tally). For the comparison reported in Figure 6, this photon directional bias has a limited impact because only 6 top detectors located right above the target materials are used, instead of the 16 top detectors shown in Figure 1 (this is another reason for the small counting statistics).

Dedicated MODAR software [56] greatly facilitates the implementation of this two-step calculation. MODAR indeed handles the F5 output file, allowing any time or energy cuts in a straightforward way. Then, for any time-energy selection of the F5 flux, it directly provides the pulse-height spectrum in a set of detectors (NaI, Ge...), the response functions of which being pre-calculated with F8 calculations. These response functions are in fact the energy deposition histograms (pulse-height tally) for mono-energetic photons covering the range of interest, here between 0 and $10 \mathrm{MeV}$. MODAR not only takes into account detection efficiency, but also time and energy resolutions of both detectors and data acquisition system, as well as counting statistics for given acquisition time and neutron emission rate. Eventually, using the total count rate recorded in the whole detectors, MODAR also calculates the random background due to accidental coincidences, which may significantly influence statistical fluctuations of the useful signal, depending on its level with respect to true coincidences.

Back to uncertainties, count losses in the data acquisition system (DAQ) and data filtering algorithms (explanation below) are also to be considered for a quantitative comparison between calculation and experiment. Count losses in electronics were limited here by using a low emission rate of the neutron generator, that is, $10^{7} \mathrm{~s}^{-1}$ (overall isotropic emission, among which approximately $1 \%$ of tagged neutrons). Consequently, EURITRACK front-end electronics only had to process less than $3000 \mathrm{~s}^{-1}$ alpha-gamma coincidences. The DAQ dead-time 

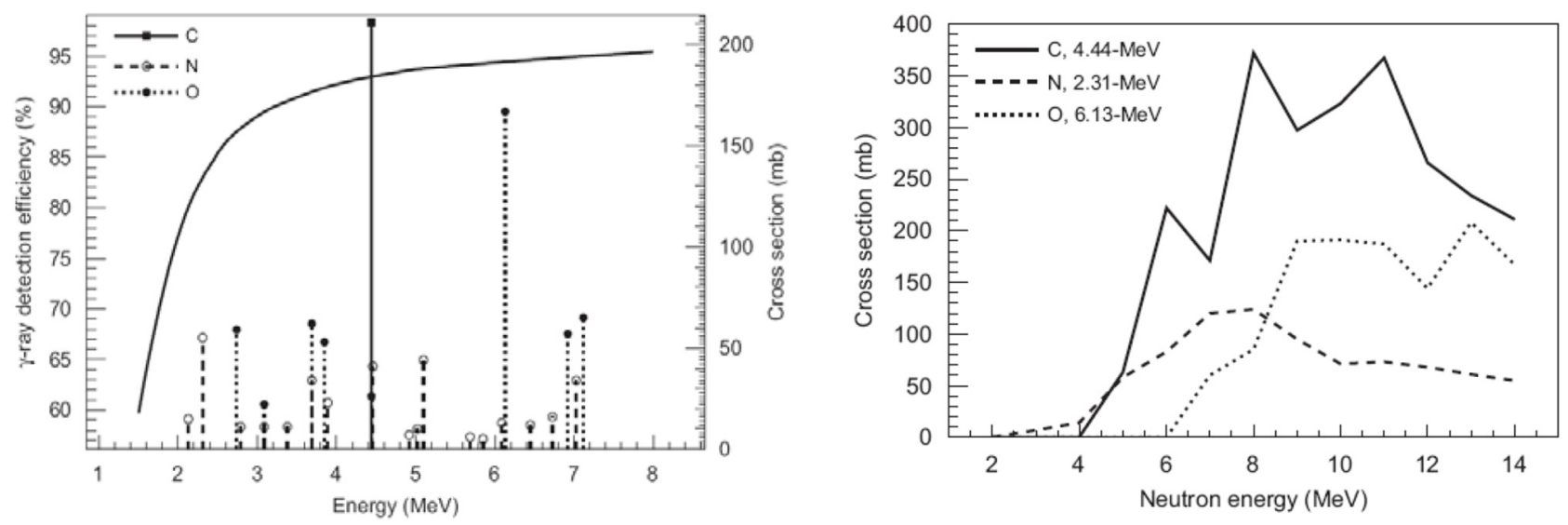

Fig. 7. $\mathrm{NaI}(\mathrm{Tl})$ detection efficiency and $\mathrm{C}, \mathrm{N}, \mathrm{O}$ gamma-ray production cross sections (see text for details).

being close to $5 \mu \mathrm{s}$, essentially due to the QDC conversion time [42], count losses are estimated lower than $1.5 \%$. On the other hand, a filtering algorithm is used to discard multiple alpha or gamma hits, and useless events recorded by the DAQ. The filtering ratio between the accepted and total number of events is here significant, typically $50 \%$, the number of accepted events being used to scale calculations. However, a fraction of rejected events could lead to real events, such as the coincidence between an alpha particle and two cascade gamma rays induced by the tagged neutron. Among the abovementioned 50\% filtered events, however, less than $10 \%$ are due to the criterion on gamma multiplicity. Therefore, cascade gamma rays, which lie in these $10 \%$ of $50 \%$ rejected events, are not liable to introduce a bias larger than a few percent.

Overall, we estimate to $20 \%$ the relative standard deviation of systematic uncertainties, that is, due to possible biases. This might be underestimated for specific gamma rays with doubtful or clearly mistaken nuclear data, but on the contrary overestimated for well-known gamma rays like the $4.439 \mathrm{MeV}$ transition of ${ }^{12} \mathrm{C}$. Within this uncertainty, the agreement between calculation and experiment is quite correct, especially when counting statistics are sufficient like for graphite $(\mathrm{C})$ and wood $\left(\mathrm{H}_{31} \mathrm{C}_{22} \mathrm{O}_{12}\right)$ spectra. Melamine $\left(\mathrm{H}_{6} \mathrm{C}_{3} \mathrm{~N}_{6}\right)$ and water $\left(\mathrm{H}_{2} \mathrm{O}\right)$ spectra suffer from larger fluctuations, but calculation still correctly reproduces the main peaks. In addition, possible biases are expected to have a limited influence when calculating fractions of counts for the $\mathrm{C}, \mathrm{N}$ and $\mathrm{O}$ elements, as detailed in next section.

\subsubsection{Conversion of $\mathrm{C}, \mathrm{N}$, and $\mathrm{O}$ counts into chemical proportions}

As mentioned above, it is necessary to convert the C, N, and $\mathrm{O}$ count fractions provided by the unfolding algorithm into chemical proportions. Therefore, conversion factors have been established for a series of cargo materials and suspicious item positions [17]. The counts attributed by the unfolding algorithm to $\mathrm{C}, \mathrm{N}$ and $\mathrm{O}$ elements, respectively, are proportional to the respective numbers of $\mathrm{C}, \mathrm{N}$, and $\mathrm{O}$ nuclei in the inspected material. The proportionality coefficients, respectively noted $\tau_{\mathrm{C}}, \tau_{\mathrm{N}}$ and $\tau_{\mathrm{O}}$, are calculated with the following equation:

$$
\begin{aligned}
& \tau_{C, N, O}\left(E_{\gamma}, Z_{e f f}, \rho, x\right) \\
& =\int_{E_{n}} \sum_{E_{\gamma}>E_{0}} \sigma_{C, N, O}\left(E_{\gamma}, E_{n}\right) \varphi\left(E_{n}\right) e^{-\mu\left(Z_{e f f}, E_{\gamma}\right) \rho x} \varepsilon\left(E_{\gamma}\right) d E_{n}
\end{aligned}
$$

where

- $E_{n}$ and $E_{\gamma}$ are respectively the incident neutron (continuous spectrum, see Fig. 8) and induced gammaray (discrete lines, see Fig. 7) energies $(\mathrm{MeV})$;

- $E_{0}$ is the low-energy threshold of the data acquisition system $(\mathrm{MeV})$;

$-\varphi\left(E_{n}\right)$ is the spectral fluence rate at the position of the inspected voxel $\left(\mathrm{cm}^{-2} \cdot \mathrm{s}^{-1}\right)$, which can be calculated with MCNP using the "F5 tally" (fluxat a point, in $\mathrm{cm}^{-2}$ per source neutron unit) multiplied by the $14 \mathrm{MeV}$ tagged neutron emission: an example of such spectral calculations is given below in Figure 8 (normalized spectra); - $\sigma_{C, N, O}\left(E_{\gamma}, E_{n}\right)$ is the differential production cross section for given $\mathrm{E}_{\gamma}$ and $\mathrm{E}_{\mathrm{n}}$ energies $\left(\mathrm{cm}^{2}\right)$;

$-\varepsilon\left(E_{\gamma}\right)$ is the intrinsic detection efficiency of $\mathrm{NaI}(\mathrm{Tl})$ detectors at energy $\mathrm{E}_{\gamma}$, that is, the number of counts above $E_{0}$ threshold (full-energy peak plus partial-energy depositions due to the escape of annihilation and Compton scattered photons) per incident photon, which can be calculated with MCNP using the "F8 tally" (pulse height distribution in a detector); the geometric detection efficiency is not described as it is the same for all elements;

- $Z_{\text {eff }}, \quad \rho$, and $\mu\left(Z_{\text {eff, }} E_{\gamma}\right)$ are respectively the effective atomic number, density $\left(\mathrm{g} . \mathrm{cm}^{-3}\right)$, and mass attenuation coefficient $\left(\mathrm{cm}^{2} \cdot \mathrm{g}^{-1}\right)$ of the inspected materials;

$-x$ is the cargo material thickness $(\mathrm{cm})$ crossed by a gamma ray from its production point towards $\mathrm{NaI}(\mathrm{Tl})$ detectors.

Multiplying the measured $\mathrm{C} / \mathrm{N}$ and $\mathrm{C} / \mathrm{O}$ count ratios (from the unfolding algorithm) by $\tau_{\mathrm{N}} / \tau_{\mathrm{C}}$ and $\tau_{\mathrm{O}} / \tau_{\mathrm{C}}$, respectively, allows recovering the corresponding nuclei ratios, that is, the chemical proportions. Figure 7 reports 

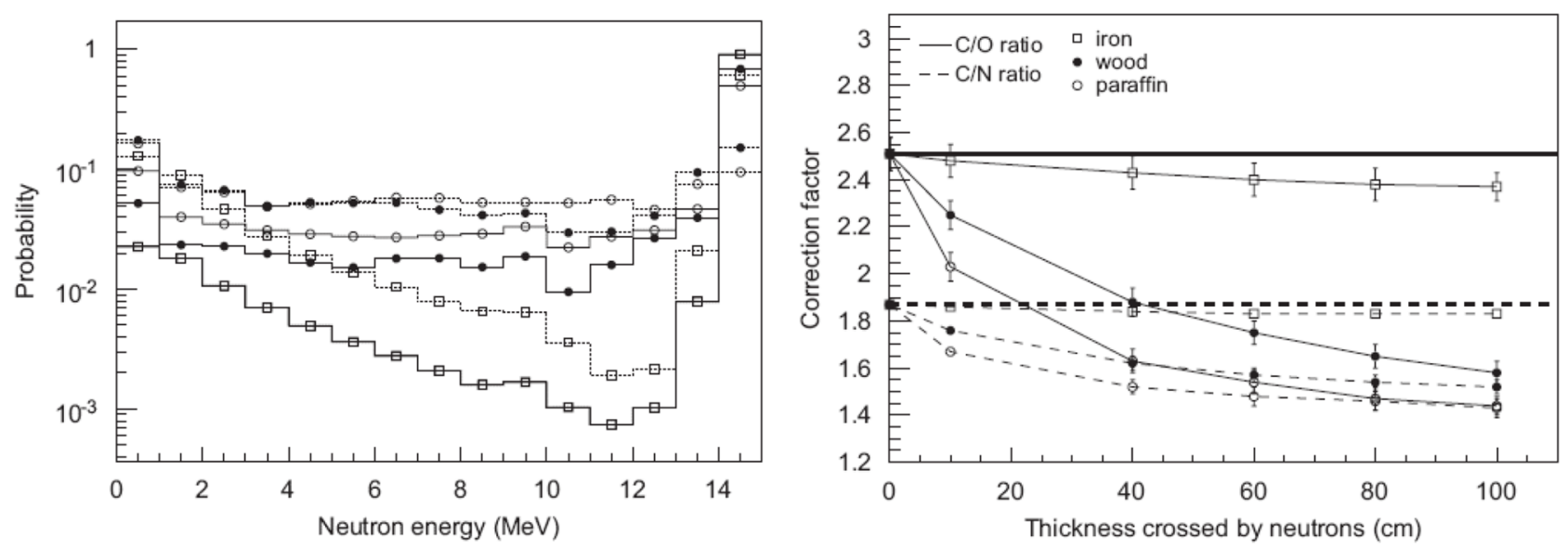

Fig. 8. Neutron energy spectra and conversion factors for different materials (see text for details).

the intrinsic detection efficiency $\varepsilon\left(\mathrm{E}_{\gamma}\right)$ calculated with MCNP for photons impinging normally the small surface of a $5^{\prime \prime} \times 5^{\prime \prime} \times 10^{\prime \prime} \mathrm{NaI}(\mathrm{Tl})$ crystal, and with a $1.35 \mathrm{MeV}$ lowenergy threshold (continuous full line). The C, N, and O gamma rays are shown as Dirac lines with pulse heights corresponding to their production cross sections for $14 \mathrm{MeV}$ neutrons. Some gamma-ray production cross sections used in MCNP (here from ENDF/B-VI.0 library) are also given in Figure 7 for major $\mathrm{C}, \mathrm{N}$, and $\mathrm{O}$ lines, as a function of neutron energy. As $\mathrm{C} / \mathrm{N}$ and $\mathrm{C} / \mathrm{O}$ conversion factors depend on neutron energy, the neutron spectrum in the position of the inspected voxel must be taken into account.

Figure 8 shows the slowed down spectra (MCNP simulations) of the original $14 \mathrm{MeV}$ tagged neutron beam inside different cargo materials, when it crosses $10 \mathrm{~cm}$ (full lines) and $100 \mathrm{~cm}$ (dotted lines) of $0.7 \mathrm{~g} . \mathrm{cm}^{-3}$ wood (full circles), $0.88 \mathrm{~g} \cdot \mathrm{cm}^{-3}$ paraffin (empty circles) and $0.5 \mathrm{~g} \cdot \mathrm{cm}^{-3}$ iron (squares). The most efficient process to reduce neutron energy being elastic scattering on hydrogen nuclei, these materials (iron, wood, paraffin) represent extreme situations in terms of neutron moderation. Figure 8 also reports the $\mathrm{C} / \mathrm{O}$ and $\mathrm{C} / \mathrm{N}$ conversion factors as a function of material thickness crossed by the neutrons. The thickness crossed by the gamma rays is here $150 \mathrm{~cm}$. The horizontal lines show the conversion factors without gamma attenuation and neutron moderation.

We used the data acquired on real cargo containers during EURITRACK project to test the validity of these conversion factors [46]. Figure 9 presents examples of flight-path spectra (neutron interactions along the neutron beam) and the induced gamma spectra for different types of cargo materials. The random background areas in the flight-path spectra are diagonally dashed and the areas related to the containers are horizontally dashed. The peak near $0 \mathrm{~mm}$ corresponds to the interactions in the neutron generator walls and in surrounding materials. The gammaray spectra associated to the abovementioned container areas are drawn in full lines, and the unfolding fit results in dashed lines.

Table 1 shows that the conversion factors allow determining $\mathrm{C} / \mathrm{O}$ chemical fractions consistent with declared cargo materials.

\subsubsection{Complete data processing}

After this original, semi-analytical approach, the calculation of correction factors has been refined to take into account the anisotropy of gamma-ray production (see Fig. 10) described in ENDF/B-VII.0 and JEF3.1 nuclear databases, but not in ENDF/B-VI.0 used above. Accordingly, it became necessary to take into account the position of NaI detectors in the EURITRACK system and therefore, the new conversion factors have been entirely calculated based on MCNP point detector (F5 tally) calculations and MODAR software post-processing [18].

The new correction factors have been established for any inspection positions inside different containers with densities varying from $0 \mathrm{~g} . \mathrm{cm}^{-3}$ (air) to $1 \mathrm{~g} . \mathrm{cm}^{-3}$ (water, most penalizing case), see Figure 11. It must be noted that the most probable and average densities for the goods transported in cargo containers are 0.1 and $0.2 \mathrm{~g} \cdot \mathrm{cm}^{-3}$, respectively, and that few commodities have densities larger than 0.6 g.cm $\mathrm{cm}^{-3}[57,58]$.

These conversion factors have been validated with bare samples of well-known materials (wood, paper, melamine, ammonium acetate), see Figure 12 [18]. The $\mathrm{O} / \mathrm{C}$ and $\mathrm{N} / \mathrm{C}$ chemical ratios, obtained from the gamma spectrum unfolded count ratios multiplied by the conversion factors, are used to build the triangle representation. On the other hand, statistical and systematic uncertainties on these relative $\mathrm{C}, \mathrm{N}$, and $\mathrm{O}$ proportions are combined using a Monte Carlo approach. Systematic errors have been assessed by simulating a large series of gamma-ray spectra (as presented in Sect. 2.1.7) corresponding to different inspection configurations (TNT and cocaine targets hidden in different positions inside cargo containers filled with iron, ceramic and organic matrixes). Systematic error intervals correspond to the observed biases between the chemical proportions deduced from the simulated gamma spectra and conversion factors, and the real chemical compositions of TNT and cocaine. The estimated error range of the $\mathrm{O} / \mathrm{C}$ and $\mathrm{N} / \mathrm{C}$ conversion factors for the iron and silicon-based (ceramics) matrixes are $\pm 20 \%$ and $\pm 35 \%$, respectively. For the wood matrix, they are $\pm 25 \%$ and $\pm 40 \%$, respectively. In the case of bare 

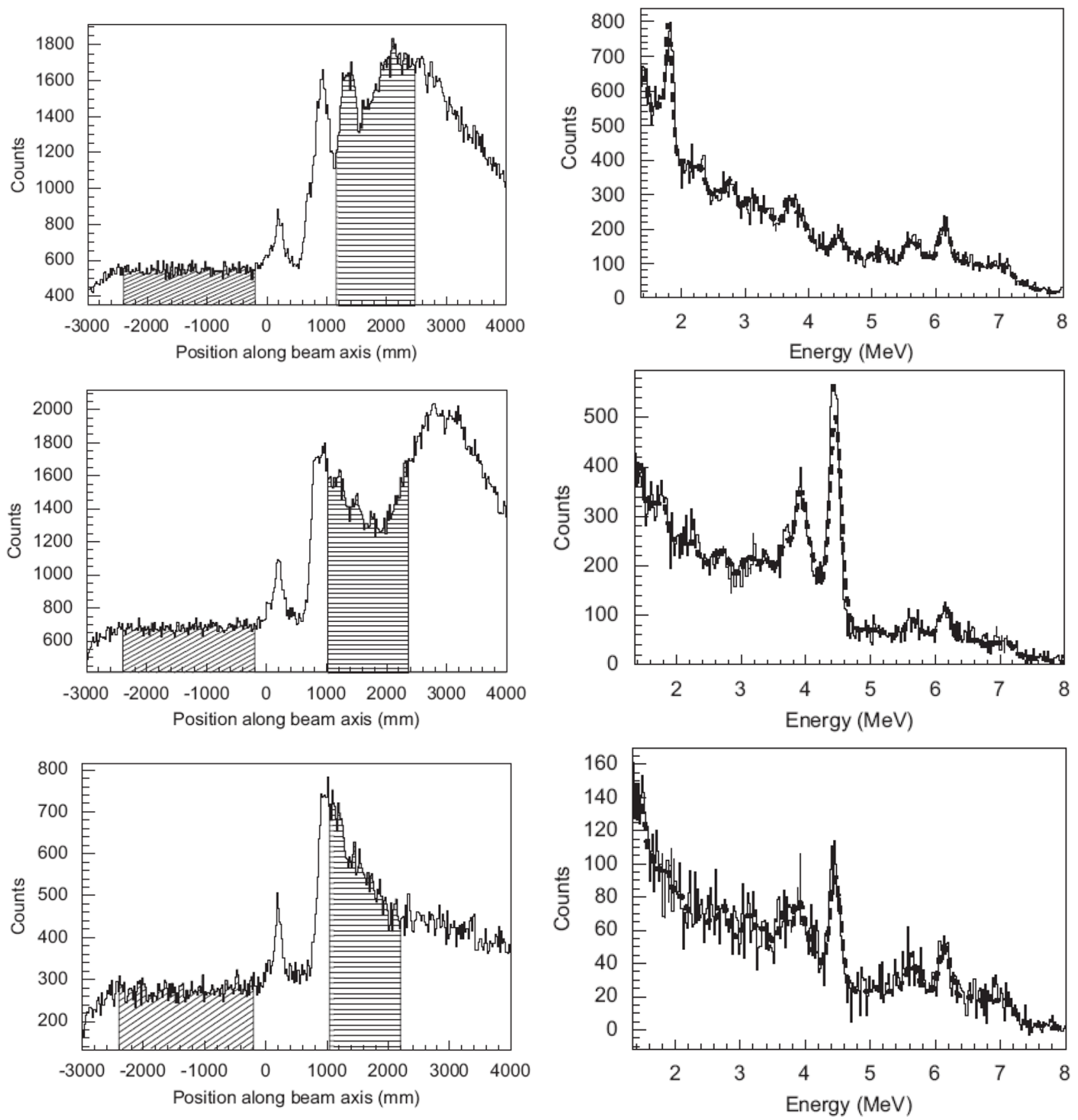

Fig. 9. Tagged neutron flight-path spectra (on the left) and gamma-ray spectra (on the right) in 10 min inspections of real containers transporting glass (on top), plastic aqua shoes (in the middle), and paper (below). See text for more details.

targets (without cargo cover load), as in Figure 12, systematic error intervals are $\pm 10 \%$ and $\pm 20 \%$ for the $\mathrm{O} / \mathrm{C}$ and $\mathrm{N} / \mathrm{C}$ conversion factors, respectively.

As observed on the triangles, the agreement between measured and expected chemical compositions is satisfactory for the above samples of wood(chemical composition is variable among species, the one adopted here is $\left.\mathrm{H}_{31} \mathrm{C}_{22} \mathrm{O}_{12}\right)$, melamine $\left(\mathrm{C}_{3} \mathrm{H}_{6} \mathrm{~N}_{6}\right)$ and ammonium acetate $\left(\mathrm{C}_{2} \mathrm{O}_{2} \mathrm{NH}_{7}\right)$. As a reminder (text following Fig. 3), the distances between any point of the triangle and each side correspond to the relative proportions of $\mathrm{C}$ (perpendicular projection on $\mathrm{O}-\mathrm{N}$ side), $\mathrm{N}$ (projection on $\mathrm{C}-\mathrm{O}$ side) and $\mathrm{O}$ (projection on $\mathrm{C}-\mathrm{N}$ side). To go further in data processing validation, these targets were placed in the $0.2 \mathrm{~g} . \mathrm{cm}^{-3}$ iron matrix shown in Figure 3. Results are reported below in Figure 13. The presence of the wood sample in the centre of the container, which is the most penalizing position, is visible between 30 and 40 ns (red lines) in the time (TOF) spectrum. Intense iron peaks dominate the gamma spectrum corresponding to this TOF window because neutron scattering in the iron cargo deviates part of tagged neutrons from the wood target. Nevertheless, C and O 
Table 1. Comparison between the expected and measured $\mathrm{C} / \mathrm{O}$ ratios for real commercial containers homogeneously filled with organic cargo materials.

\begin{tabular}{llll}
\hline Truck \# & Material & Real C/O & Measured C/O \\
\hline $\mathrm{I}$ & Wood & 1.83 & $1.9 \pm 0.4$ \\
2 & Cotton & 1.39 & $1.5 \pm 0.2$ \\
4 & Wood & 1.83 & $1.7 \pm 0.5$ \\
10 & Wood & 1.83 & $1.9 \pm 0.4$ \\
11 & Cotton & 1.39 & $1.4 \pm 0.3$ \\
15 & Wood & 1.83 & $2.0 \pm 0.4$ \\
16 & Cotton & 1.39 & $1.7 \pm 0.2$ \\
17 & Wood & 1.83 & $1.8 \pm 0.1$ \\
31 & Cotton & 1.39 & $1.6 \pm 0.2$ \\
33 & Wood & 1.83 & $1.8 \pm 0.3$ \\
34 & Polyester & 3.00 & $3.2 \pm 0.2$ \\
36 & Polyester & 3.00 & $2.7 \pm 0.2$ \\
44 & Wood & 1.83 & $1.7 \pm 0.3$ \\
51 & Paper & 1.20 & $1.2 \pm 0.2$ \\
\hline
\end{tabular}

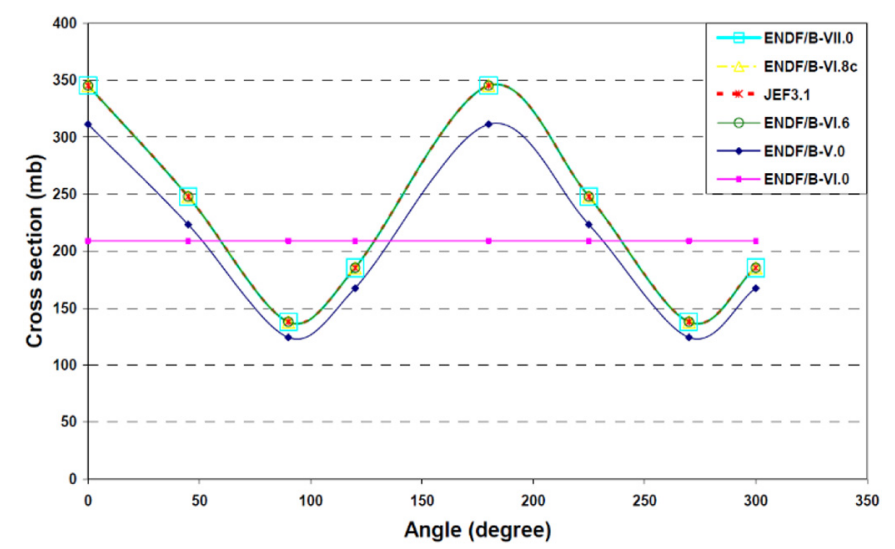

Fig. 10. Angular distribution of the gamma-ray production cross section in different nuclear databases for the $4.443 \mathrm{MeV}$ line of carbon induced by $14 \mathrm{MeV}$ neutrons.

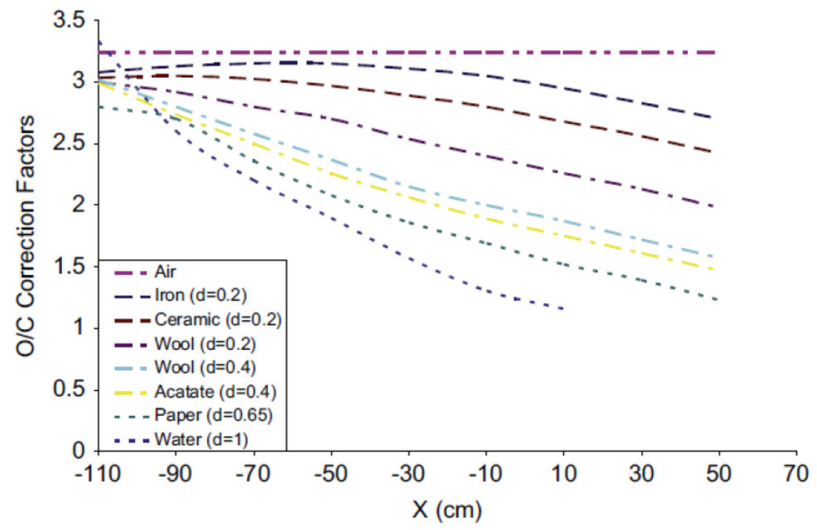

peaks are still visible and measurement provides an $\mathrm{O} / \mathrm{C}$ chemical ratio of $0.51 \pm 0.17$, that is, close to that of wood (0.54).

Figure 14 shows the results for the melamine and ammonium acetate targets placed in the middle of the same iron matrix. Compared to Figure 12 with bare samples, the uncertainty around the measured points is larger, due to increased counting statistical fluctuations and systematic errors, but the position on the triangle is correct.

Eventually, in order to reduce the statistical uncertainties on elemental detection by the unfolding algorithm, especially nitrogen, synthetic spectra have been generated with a Monte Carlo approach. A synthetic spectrum is generated from the measured energy spectrum by randomly sampling every bin contents according to a Gaussian distribution centred on the recorded number of counts and with a standard deviation based on Poisson law (root square of the number of counts). For each measured energy spectrum, 300 synthetic spectra are thus generated and unfolded to build 300 sets of count proportions, which provides for each element a count fraction distribution. These distributions are fitted with Gaussian functions, the mean of which $\mathrm{Pi}$ is used as the most probable count fraction of element $i$ with a standard deviation $\sigma i$. If the distribution is too broad, the element is considered as absent from the gamma-ray spectrum of the inspected cargo container. This is illustrated in next section.

\subsubsection{Inspection of real containers}

Nearly 150 real cargo container inspections have been performed in the seaport of Rijeka, Croatia, during the EURITRACK and Eritr@C following projects. The TOF and energy spectra of a real container transporting wood materials (declared as "bamboo poles" in the cargo manifest) are presented in Figure 15. By using the unfolding algorithm on the measured energy spectrum, no nitrogen was detected and the measured $\mathrm{O} / \mathrm{C}$ ratio $(0.59 \pm 0.20)$ is in good agreement with the wood chemical ratio, but with a large uncertainty. Figure 16 shows that uncertainties are improved thanks to the synthetic spectra method. The $\mathrm{O} / \mathrm{C}$ and $\mathrm{N} / \mathrm{C}$ measured ratios indeed become $0.56 \pm 0.09$ and $0.04 \pm 0.09$, respectively, and no nitrogen is

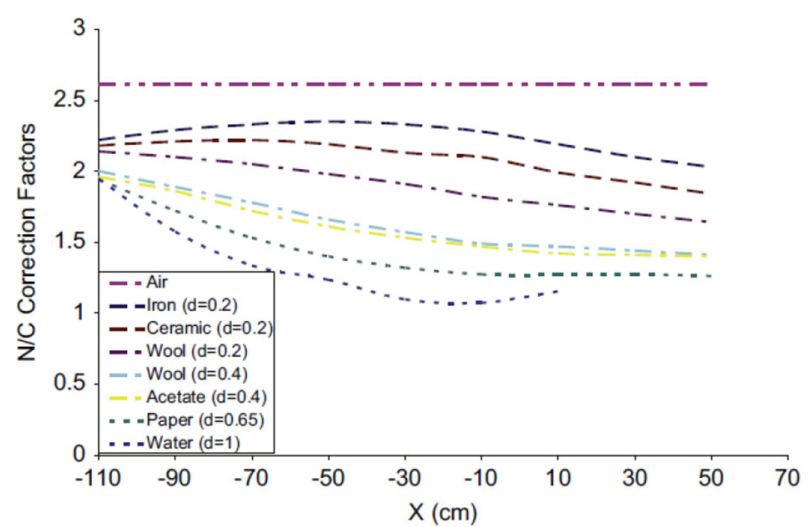

Fig. 11. O/C (left) and N/C (right) conversion factors for different cargo materials and at different depths (x) inside them, here with the tagged neutron beam at middle height of the container. 

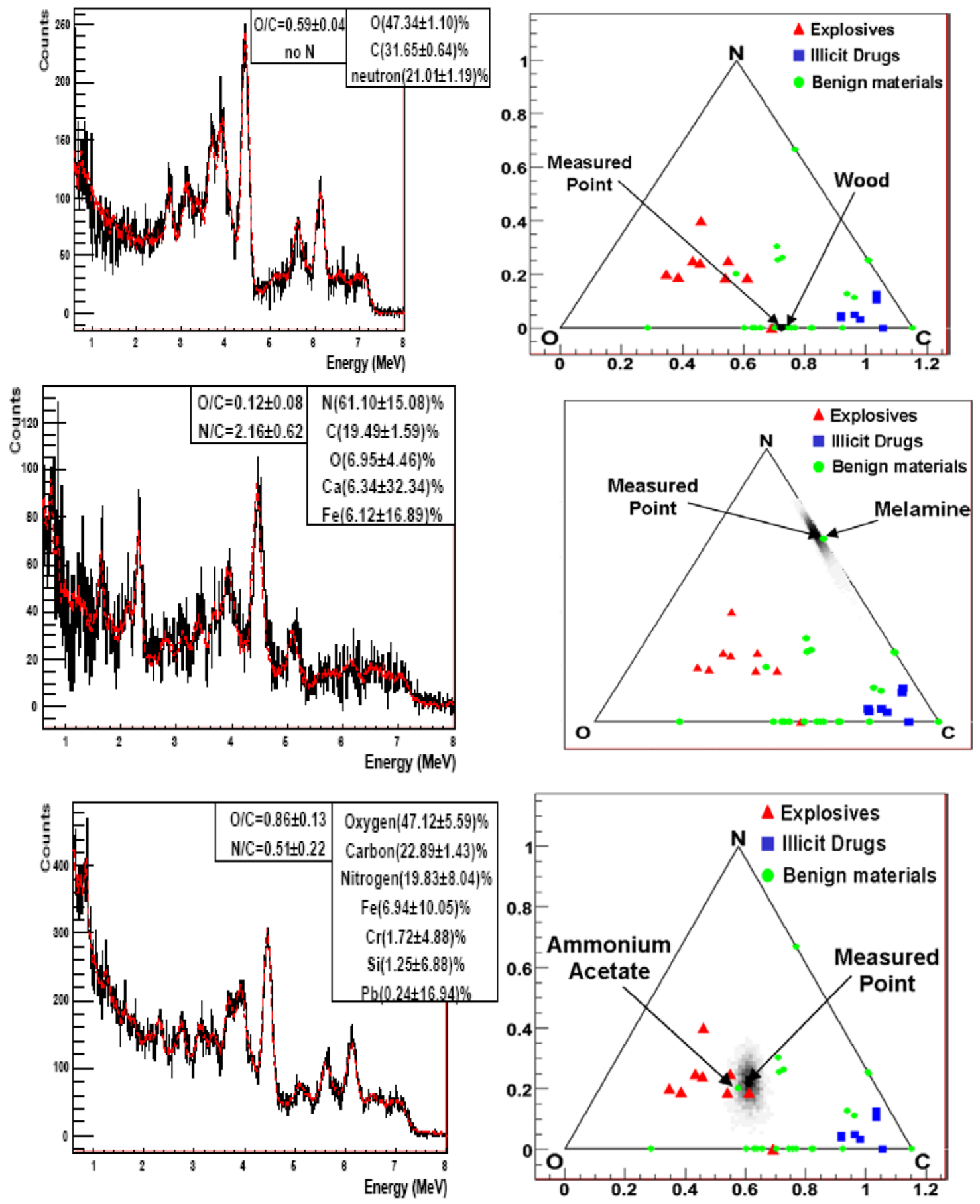

Fig. 12. Gamma-ray spectra and triangle representation of the C, N, and O chemical proportions for bare samples of wood (on top), melamine (in the middle), and ammonium acetate (below). The dashed red lines in the energy spectra correspond to the fit with a linear combination of the pure element spectra. The unfolded count fractions are given in $\%$ and the reported $\mathrm{O} / \mathrm{C}$ and $\mathrm{N} / \mathrm{C}$ are the chemical ratios obtained with the conversion factors calculated for these measurement setups.

found in $82 \%$ of the synthetic spectra. The measured point is also close to wood in the triangle diagram. As a reminder, $\mathrm{O} / \mathrm{C}$ and $\mathrm{N} / \mathrm{C}$ systematic uncertainties in the wood matrix are $\pm 25 \%$ and $\pm 40 \%$, respectively.

The triangle plot also reports the heights (in decreasing order) of explosives, narcotics and benign materials on the 2D uncertainty area, thus providing the end-user (custom, army, or police officer) with easy-to-interpret data. All heights have been normalized to the maximal height of the probability distribution (in Fig. 16, wood is found as the most probable material, with a $100 \%$ relative height). This list of most likely materials can be combined with other information to make a decision (targeting office, X-ray scan, manifest of transported goods, etc.). 

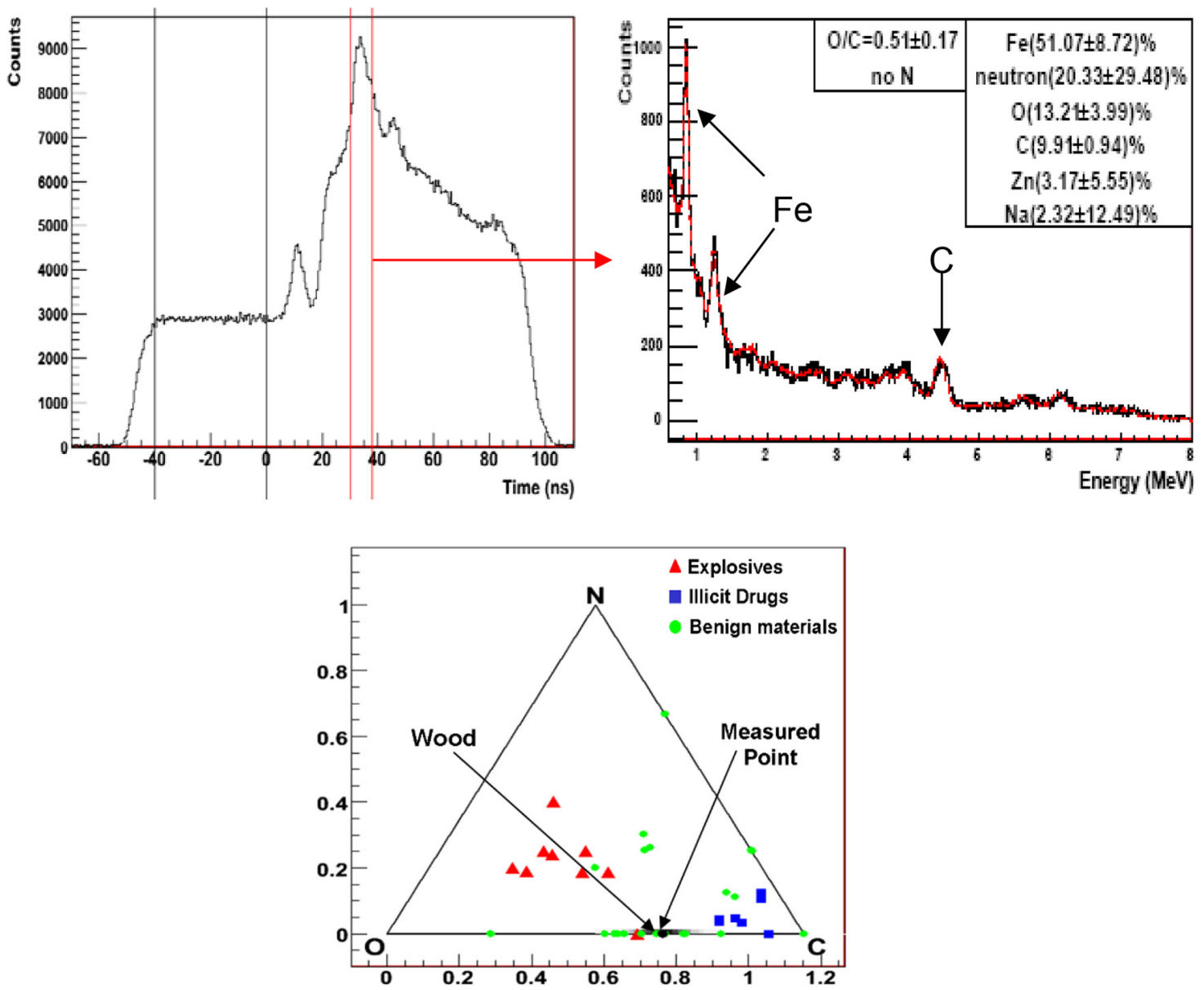

Fig. 13. TOF (top, left) and energy (top, right) spectra corresponding to the wood target hidden in the middle of the iron test matrix. The dashed red line in the energy spectrum corresponds to the fit with a linear combination of pure element spectra. Triangle diagram (below) of the carbon, nitrogen, and oxygen amounts relatively to illicit and benign materials, showing the measured point and the real wood position.

Note: the so-called "measured point" indicated on the triangle plot corresponds to the barycentre of the $2 \mathrm{D}$ probability distribution. Due to "no nitrogen" occurrences, it does not exactly match with the maximum height (here wood).

Figure 17 shows the TOF and energy spectra associated to a truck transporting clothes, where contributions of carbon, nitrogen, and oxygen are observed (but with large uncertainties for $\mathrm{O}$ and $\mathrm{N}$ ). Nitrogen seems to be present in the transported goods, as evidenced by the synthetic spectra method that significantly reduces uncertainties, see Figure 18. The measured clothes could be synthetic fibres like nylon, for which $\mathrm{O} / \mathrm{C}=\mathrm{N} / \mathrm{C}=0.166$. The barycentre diagram shows that nylon is the second most probable detected material with a height of about $20 \%$ on the $2 \mathrm{D}$ uncertainties area, the first one being Kevlar, another synthetic fibre. As previously, systematic uncertainties considered for the $\mathrm{O} / \mathrm{C}$ and $\mathrm{N} / \mathrm{C}$ conversion factors in this organic matrix are $\pm 25 \%$ and $\pm 40 \%$, respectively. Taking into account the total uncertainty area, it appears that illicit drugs (Mandrax, LSD) are also possible materials, even if their relative heights on the uncertainty surface are quite small (less than 5\%). The similarity of benign and illicit materials at the frontier of these materials area in the triangle plot is one of the limitations of the method. Ammonium acetate and TNT are also located at the border between benign and explosive materials, see for instance in Figure 3, and for this reason ammonium acetate is often used as a TNT surrogate to test the performances. Nevertheless, the objective is to provide the end-user with multiple sources of information to make a decision, which is finally based on intelligence (targeting cell), declaration of goods, X-ray scan, and neutron inspection.

\subsubsection{Simulation of explosives and narcotics detection}

The purpose of the EURITRACK system is the detection of explosives and illicit drugs in cargo containers but real trucks carrying such items were not encountered during field tests in Rijeka seaport. Experiments with real narcotics have been performed [59], but under a strong police control and without the possibility to perform multiple trials with different matrixes.

Therefore, realistic inspections have been simulated to study detection performances in 10 min acquisitions with a total neutron generator emission level of $10^{7} \mathrm{~s}^{-1}$ and 

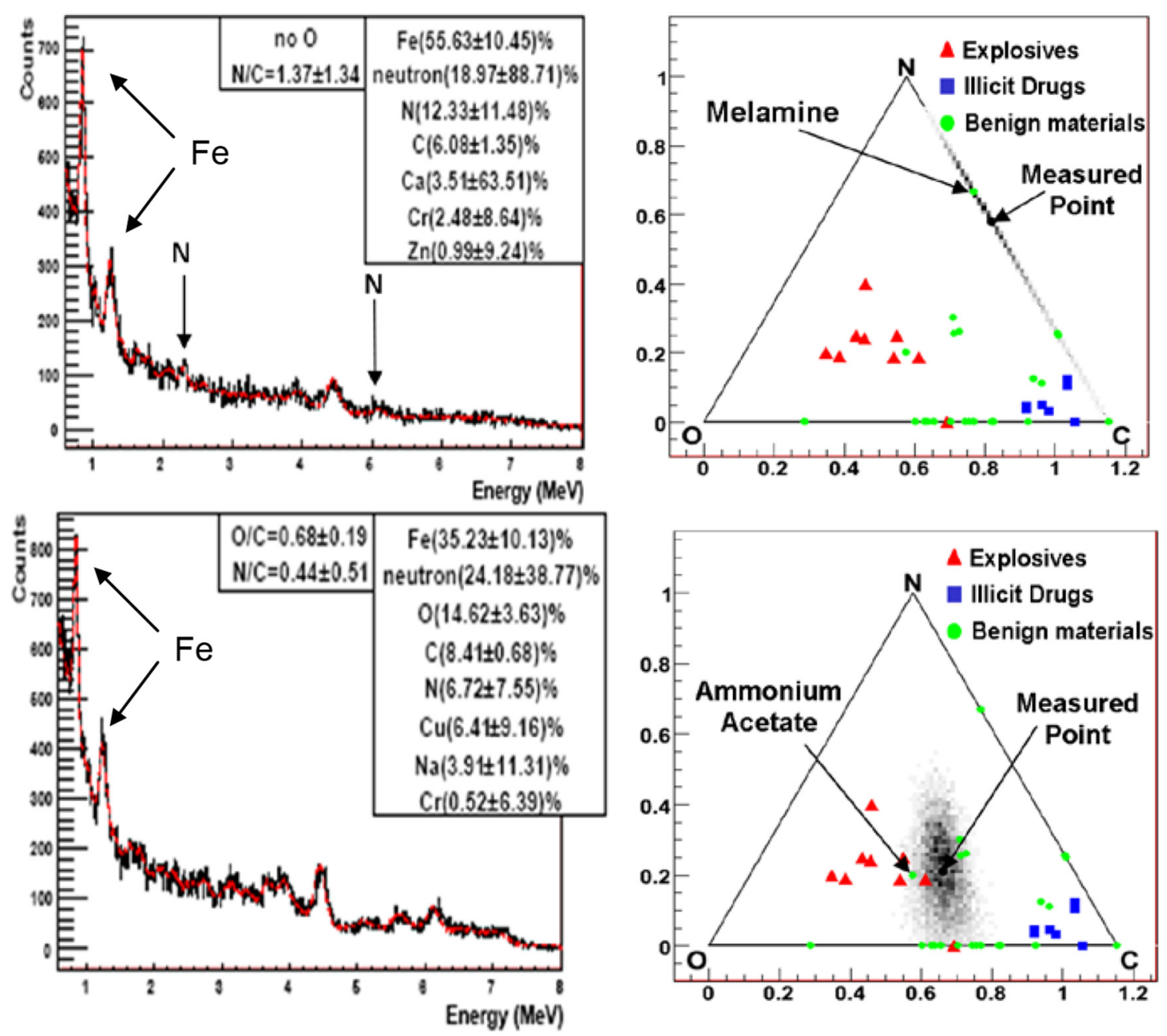

Fig. 14. Gamma-ray spectra and triangle plot of the melamine andammonium acetate samples placed in the middle of the iron matrix.
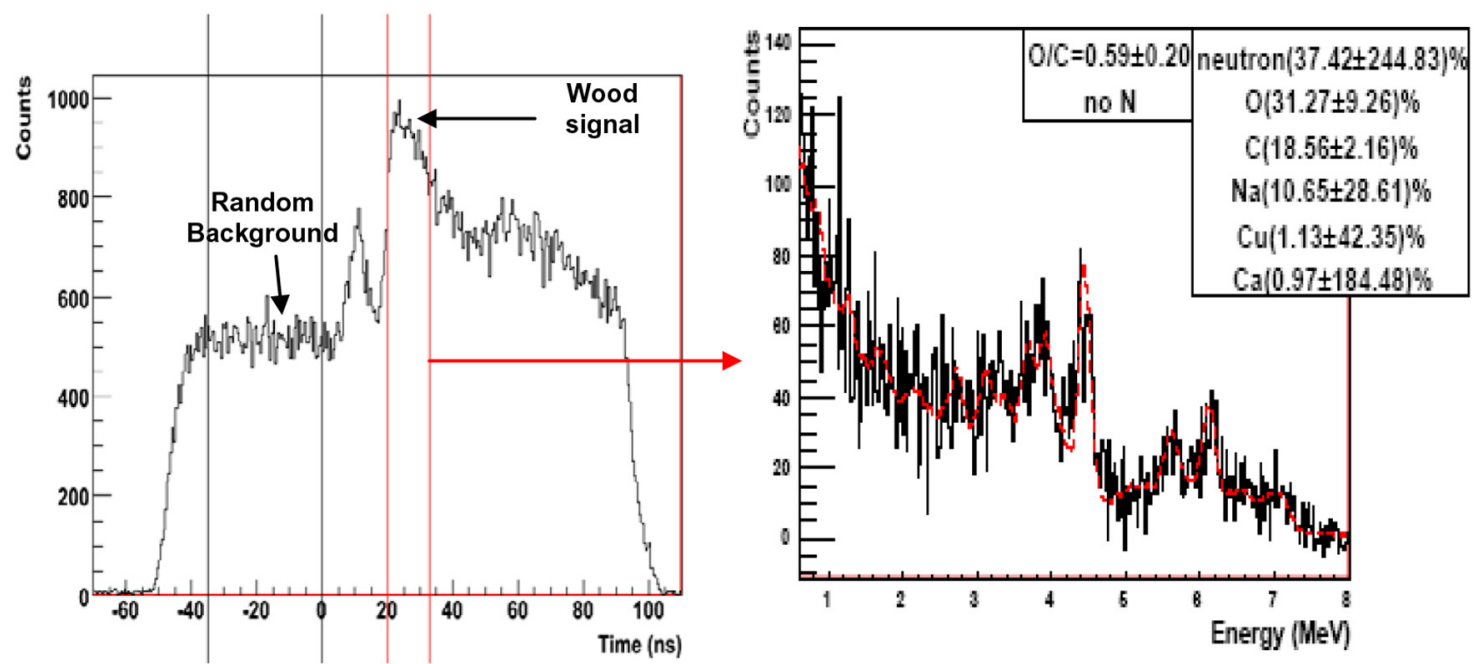

Fig. 15. TOF spectrum of a truck transporting wood (left) and gamma-ray spectrum (right) corresponding to a TOF selection marked by the red vertical lines. The random background has been subtracted to the wood energy spectrum and the dashed red line corresponds to the fit with a linear combination of pure element spectra. 

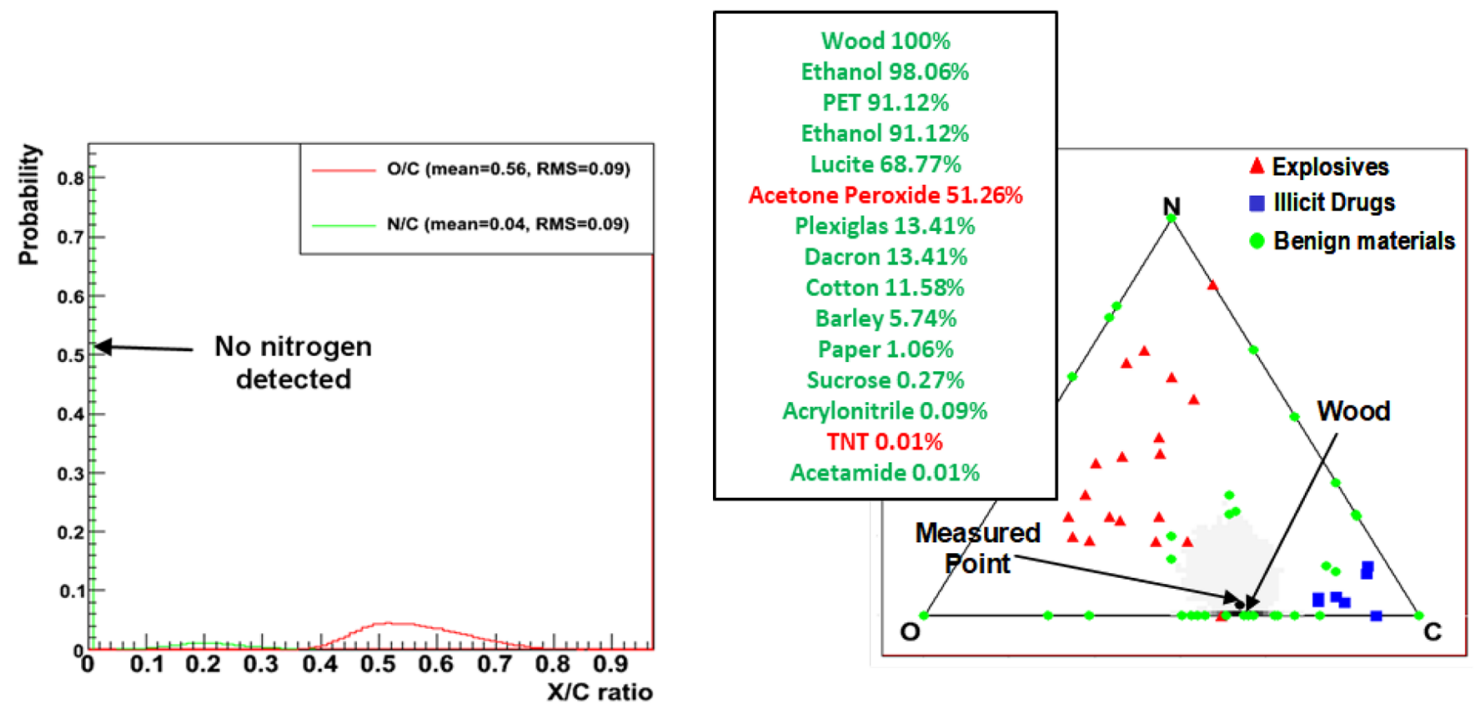

Fig. 16. On the left, $\mathrm{O} / \mathrm{C}$ and $\mathrm{N} / \mathrm{C}$ elemental ratio distributions. On the right, barycentre diagram of the $\mathrm{C}$, $\mathrm{N}$, and $\mathrm{O}$ elemental proportions of illicit and benign materials, with an indication of the most probable materials ranked by decreasing likelihood with respect to measured data.
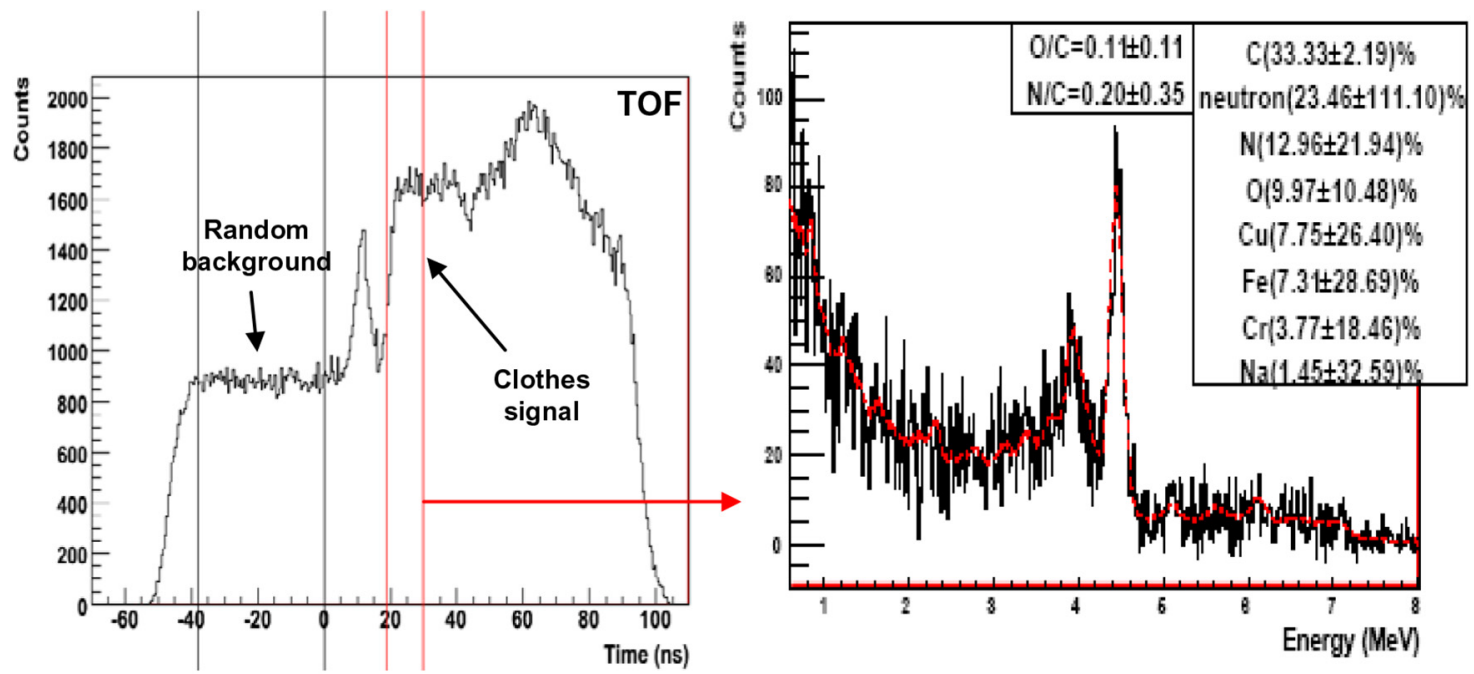

Fig. 17. TOF (left) and energy spectra (right, for the TOF selection between the red lines) of a truck transporting clothes. The random background has been subtracted and the red line in the energy spectrum corresponds to the fit with a linear combination of pure element spectra.

a tagged neutron proportion of about 1\%. Neutron emission was indeed limited in the seaport of Rijeka, Croatia, for radiological protection purposes. It was increased in next C-BORD tagged neutron inspection system by closely shielding the neutron generator, as described in Section 2.1.

The simulations reported below are intended to provide an order of magnitude of performances in view to guide future developments. Therefore, count losses in EURITRACK data acquisition electronics and processing algorithms (about 50\%, see Sect. 2.1.3) are not taken into account because they can be reduced in future systems like C-BORD. However, the physical random background due to accidental coincidences is present in the model to mimic realistic counting fluctuations.
The simulated inspections correspond to $40 \mathrm{~cm} \times 40$ $\mathrm{cm} \times 40 \mathrm{~cm}$ blocks of TNT and cocaine hidden in different positions inside containers filled with three cargo matrices representing main categories of transported goods observed during field tests in Rijeka [57]. These matrices are iron, $\mathrm{SiO}_{2}$ (glass, ceramics, fibreglass wool, etc.) and wood $\left(\mathrm{H}_{31} \mathrm{C}_{22} \mathrm{O}_{12}\right)$. Due to high neutron scattering and attenuation in rich-in-hydrogen cargo like wood (see Fig. 19), it is expected that a second neutron generator will be implemented in a future industrial system to inspect the "second half" of the container, with respect to the neutron source. A possible alternative is to select the side of the container to be inspected with by means of an advanced X-ray scanner providing depth information. Therefore, only the positions shown in Figure 19 are simulated here. 

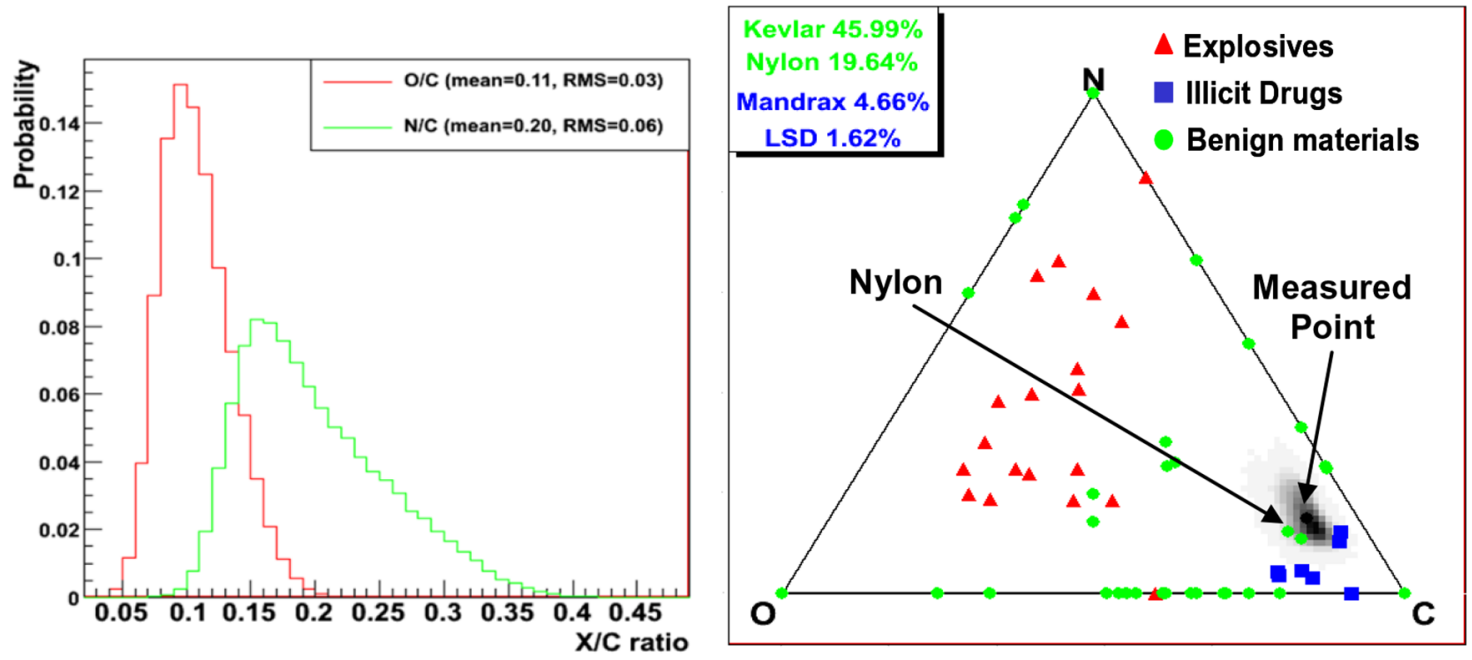

Fig. 18. On the left, distributions of $\mathrm{O} / \mathrm{C}$ and $\mathrm{N} / \mathrm{C}$ elemental ratios, and on the right barycentre diagram of the $\mathrm{C}$, $\mathrm{N}$, and $\mathrm{O}$ proportions showing that the measured point is close to nylon or Kelvar fibres.

?

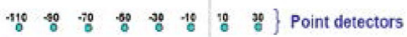

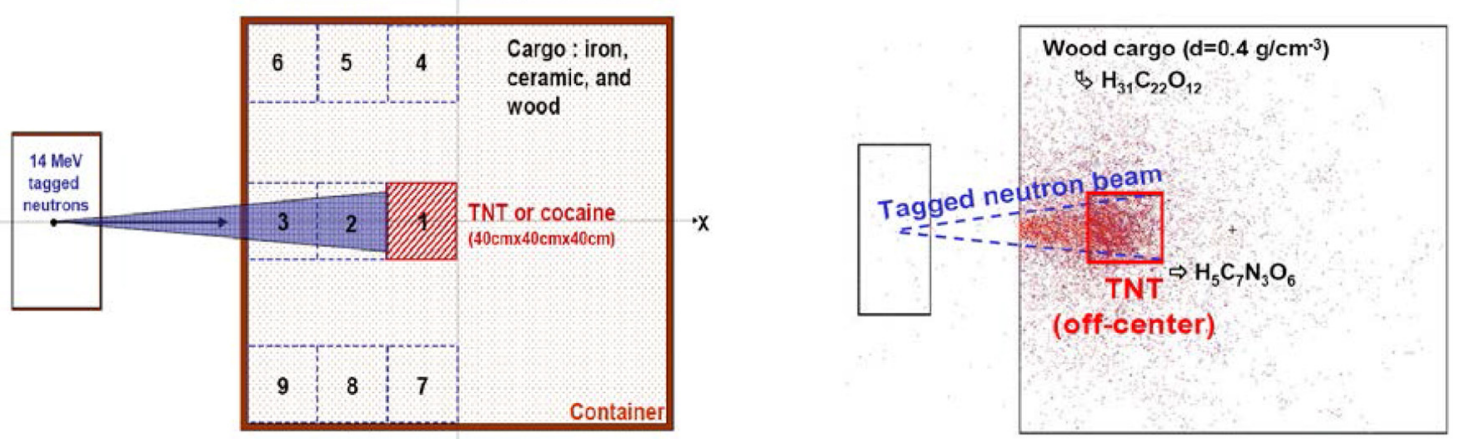

Fig. 19. On the left, simulation setup with TNT or cocaine blocks hidden inside the cargo container in nine different positions. On the right, source neutron tracking performed with the Visual Editor of MCNP (VISED) for a container filled with a wood matrix and a TNT block in position 2 .

The neutron-induced photon flux is estimated with point detectors (F5 tally of MCNP) located above the container in the position of gamma detectors and MCNP output files are processed with MODAR software [56]. Contrary to the setup shown in Figure 1, the large $5 " \times 5 " \times 10 " \mathrm{NaI}(\mathrm{Tl})$ are considered here in a horizontal instead of vertical position, to increase detection efficiency. Indeed, the lead shield around detectors intended to limit count rate and gamma cross talk could be suppressed in future systems by closely shielding the neutron generator and by using cross talk mitigation algorithms.

A simulation example is presented in Figure 20 corresponding to the $40 \mathrm{~cm} \times 40 \mathrm{~cm} \times 40 \mathrm{~cm}$ TNT block $\left(\mathrm{C}_{7} \mathrm{H}_{5} \mathrm{O}_{6} \mathrm{~N}_{3}\right)$ with a density of 1.6 g.cm ${ }^{-3}$ hidden in position 3 of Figure 19 (close to the container wall) in $\mathrm{SiO}_{2}$-based cargo materials with a density of $0.2 \mathrm{~g} . \mathrm{cm}^{-3}$. The distanceto-interaction spectrum (on top, left panel) shows a high peak corresponding to TNT and the corresponding gamma spectrum (bottom, left) shows the presence of unexpected $\mathrm{C}$ and $\mathrm{O}$ elements, instead of just $\mathrm{Si}$ and $\mathrm{O}$ (top, right) as in the next window corresponding to the $\mathrm{SiO}_{2}$ matrix. The $\mathrm{O} / \mathrm{C}$ and $\mathrm{N} / \mathrm{C}$ chemical ratios obtained from the unfolded count ratio and appropriate conversion factors are $0.80 \pm 0.12$ and $0.29 \pm 0.10$, respectively, which is compatible with TNT, as confirmed by the triangle representation (bottom, right). The systematic uncertainties associated to the $\mathrm{O} / \mathrm{C}$ and $\mathrm{N} / \mathrm{C}$ conversion factors are $\pm 20 \%$ and $\pm 35 \%$, respectively, for the $\mathrm{SiO}_{2}$ matrix.

A second example is presented in Figure 21, corresponding to a $0.8 \mathrm{~g} \cdot \mathrm{cm}^{-3}$ cocaine hydrochloride $\left(\mathrm{C}_{17} \mathrm{O}_{4} \mathrm{NH}_{22} \mathrm{Cl}\right)$ target $(40 \mathrm{~cm} \times 40 \mathrm{~cm} \times 40 \mathrm{~cm})$ hidden in position 5 (deeper inside the container but close to detectors, see Fig. 19) inside a $0.2 \mathrm{~g} . \mathrm{cm}^{-3}$ iron cargo. The distance-to-interaction spectrum (on the left) clearly evidences the presence of a dense material in the iron matrix, the gamma spectrum of which (in the middle) showing carbon, oxygen and nitrogen signatures. The $\mathrm{O} / \mathrm{C}$ and $\mathrm{N} / \mathrm{C}$ chemical ratios obtained by processing the simulated data $(0.19 \pm 0.02$ and $0.03 \pm 0.01)$ places the measured point in the region of illicit drugs in the triangle 

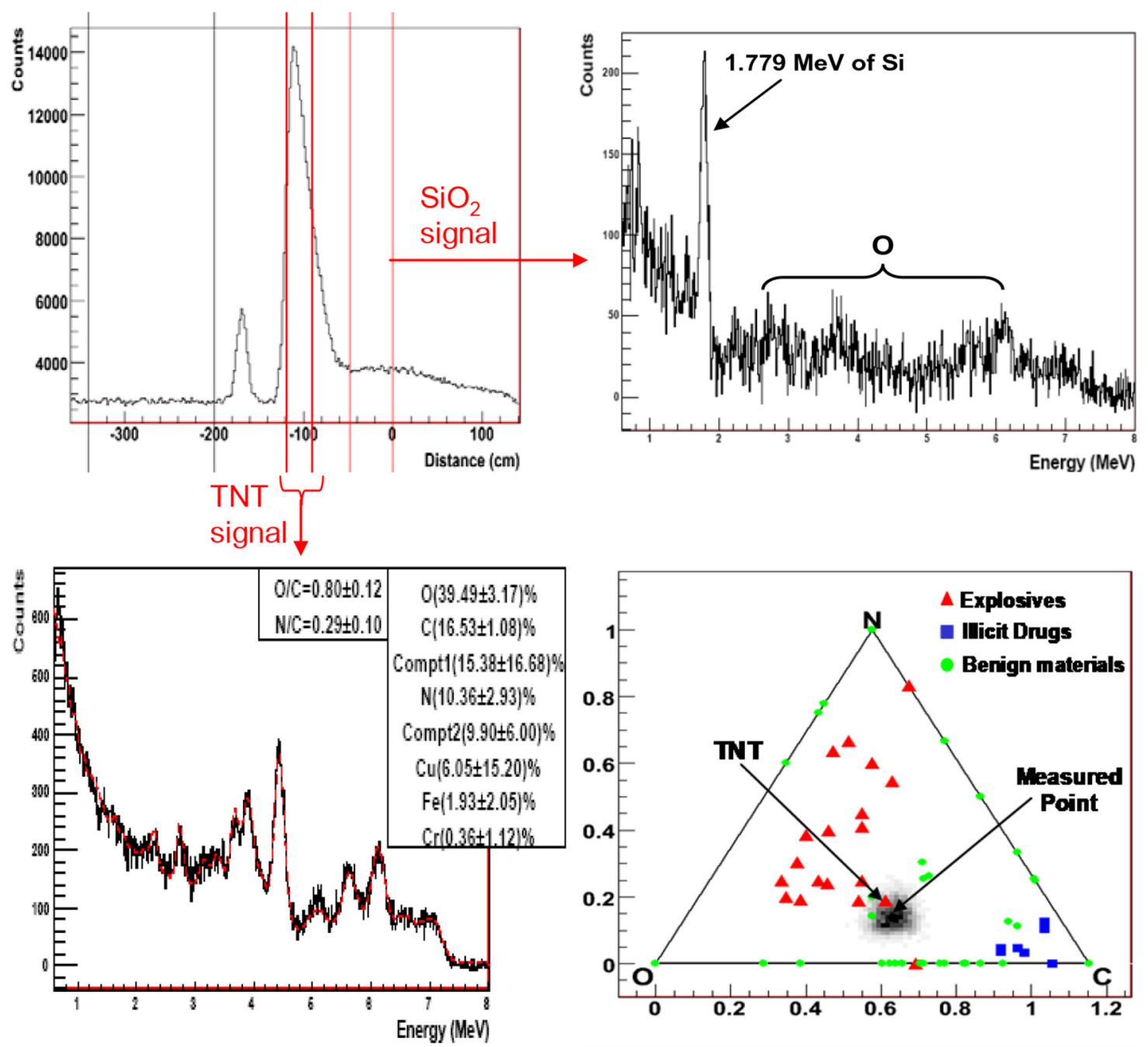

Fig. 20. Simulation inspection data of a TNT block in position 3 of Figure 19 inside a $\mathrm{SiO}_{2}$ cargo container (see text for details).
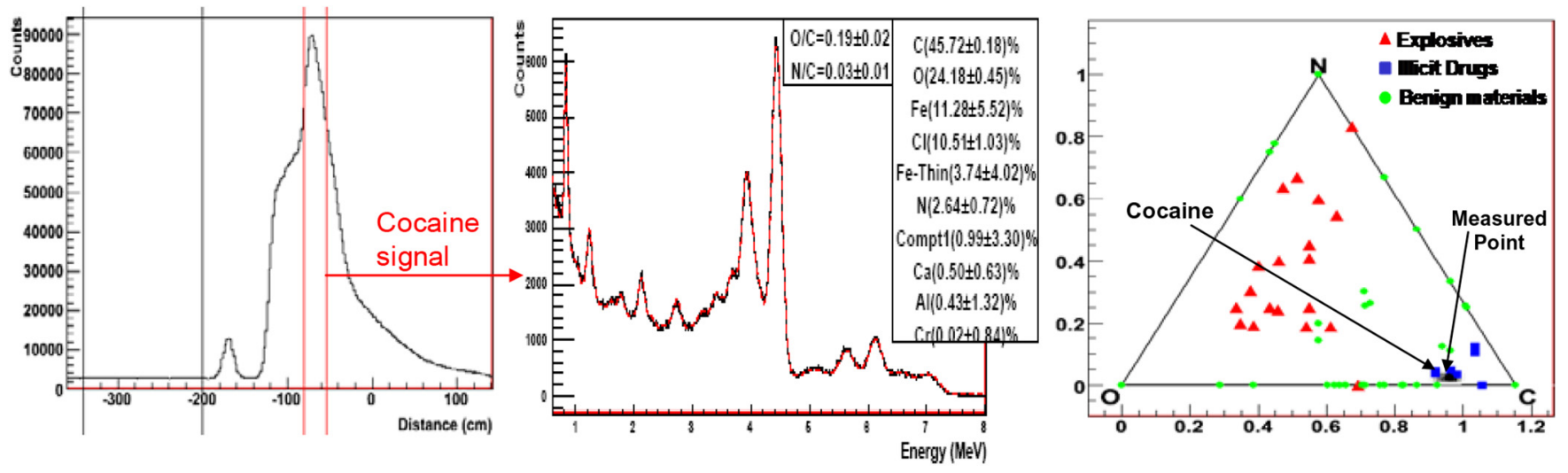

Fig. 21. Simulated inspection data of a cocaine block in position 5 (see Fig. 19) of a $\mathrm{SiO}_{2}$ cargo container (see text for details).

plot (right), with an uncertainty area (including systematic errors of $\pm 20 \%$ and $\pm 35 \%$, respectively, in the case of an iron matrix) encompassing cocaine. However, despite good counting statistics, the unfolded nitrogen count fraction suffers from a large uncertainty as it represents only a small fraction of the whole gamma spectrum. The uncertainty on unfolded count fractions of only a few $\%$ is indeed very large, and even the uncertainty given by the least square 

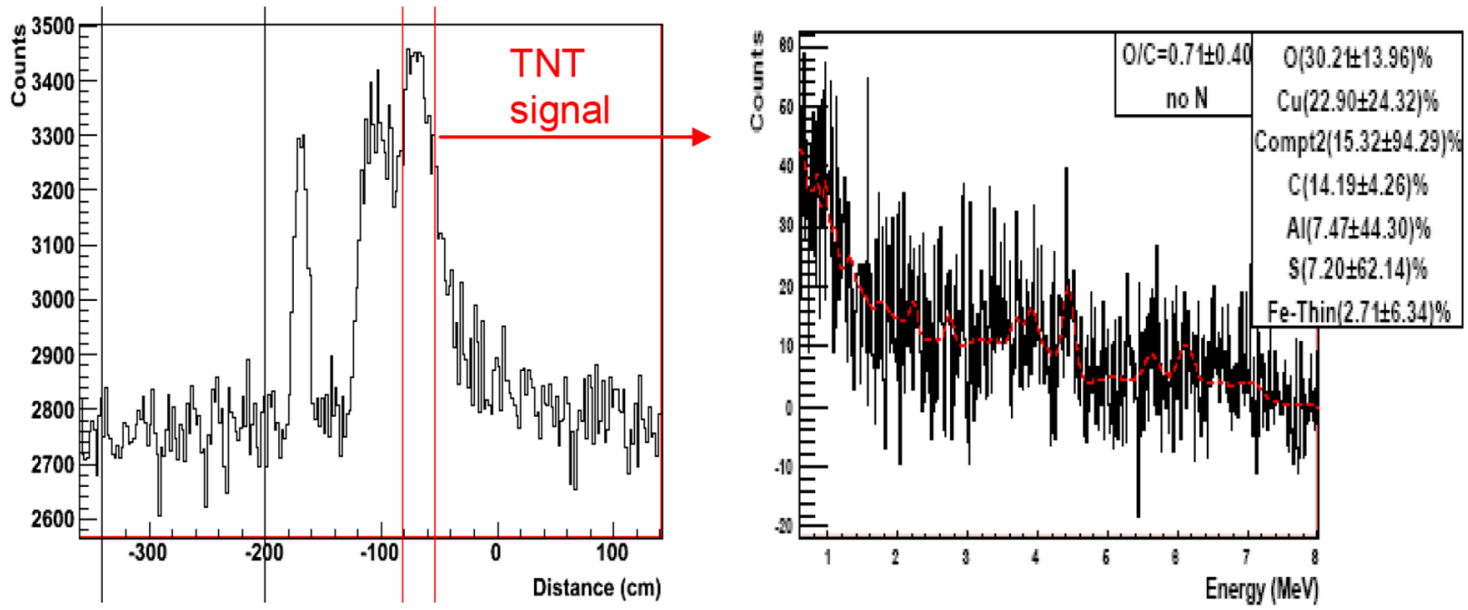

Fig. 22. Distance-to-interaction (left) and gamma-ray (right) spectra corresponding to a TNT block hidden in position 8 (see Fig. 19) inside a $0.4 \mathrm{~g} \cdot \mathrm{cm}^{-3}$ wood matrix.

algorithm is in practice underestimated as the fit includes other minor elements that are not present in the container (here $\mathrm{Ca}$ and $\mathrm{Al}$ for instance).

A last example reported in Figure 22 shows a very penalizing case with the TNT block in bottom position 8 (see Fig. 19) of a container transporting a $0.4 \mathrm{~g} . \mathrm{cm}^{-3}$ wood matrix. Counting statistics is very poor due to both neutron scattering in wood (see Fig. 19, right panel) and gamma attenuation between TNT position and top gamma detectors. The presence of the TNT block (larger density) is still visible in the distance-to-interaction spectrum, but statistical fluctuations are too large in the corresponding gamma spectrum to detect nitrogen.

Nitrogen being a key element for organic element differentiation (triangle plot), a calculation has been performed with more $5 " \times 5 " \times 10$ " $\mathrm{NaI}(\mathrm{Tl})$ top detectors than in EURITRACK system (32 vs. 16 top detectors), see Figure 23. Counting statistics is improved and nitrogen is now detected, but its count fraction is still not statically significant. The measured point is not far from TNT in the triangle diagram but the uncertainty area is still very large, mainly due to statistical fluctuations. The $\pm 25 \%$ and $\pm 40 \%$ systematic errors on the $\mathrm{O} / \mathrm{C}$ and $\mathrm{N} / \mathrm{C}$ conversion factors in a wood cargo, respectively, are here dominated by statistical uncertainties. The synthetic spectra method allows reducing the uncertainty area (bottom panels of Fig. 23), with a probability of no nitrogen detection of about $20 \%$. The obtained $\mathrm{O} / \mathrm{C}$ and $\mathrm{N} / \mathrm{C}$ chemical ratios $(0.88 \pm 0.21$ and $0.32 \pm 0.24)$ are consistent with TNT (0.86 and 0.43$)$ and the measured point is close to TNT, with a nearly $32 \%$ relative height on the $2 \mathrm{D}$ probability surface. On the other hand, other materials without nitrogen are given with a larger probability, which highlights the importance to detect nitrogen more precisely.

Implementing detectors under the truck transporting the cargo container to improve counting statistics does not appear as a practical solution, especially in view of a future transportable system. In addition, dense objects under the truck (wheels, axes, fuel tank, etc.) may significantly attenuate gamma rays and complicate data analysis.
An alternative would be to use detectors in "reflection" position, that is, close to the neutron generator as shown in Figure 1. Those reflection detectors could not be used in EURITRACK because of insufficient shielding, and therefore, too large total count rate and random background. Figure 24 shows a simulation with ten 5 " $\times 5$ " $\times 10$ " $\mathrm{NaI}(\mathrm{Tl})$ detectors located near the neutron generator, considered appropriately shielded as in C-BORD system (see next section), again for the TNT target in position 8 inside a wood cargo. Counting statistics is now two decades larger than with the 32 top detectors. Specific $\mathrm{O} / \mathrm{C}$ and $\mathrm{N} / \mathrm{C}$ conversion factors have been calculated for this inspection setup (reflection detectors) and systematic uncertainties have been estimated to be $\pm 30 \%$ and $\pm 45 \%$, respectively. The obtained $\mathrm{C}, \mathrm{N}$, and $\mathrm{O}$ elemental proportions are in good agreement with TNT $\left(\mathrm{C}_{7} \mathrm{H}_{5} \mathrm{O}_{6} \mathrm{~N}_{3}\right)$. As statistical fluctuations are very small, the shape of the uncertainty area in the triangle plot is due to these systematic uncertainty intervals.

The major improvement identified during EURITRACK project is to increase counting statistics for inspections in the lower parts of the containers. Another improvement would be the capability to move and operate rapidly in different sites (seaports, border control points, etc.), with a compact neutron generator shielding, to limit the dose to operators or non-radiation workers and the size of the restricted area. Therefore, an entirely new design has been proposed in the C-BORD European project (H2020) presented in next section.

\subsubsection{C-BORD rapidly relocatable tagged neutron inspection system}

To offer more operational flexibility, the EURITRACK design evolved towards the relocatable and compact C-BORD Rapidely Relocatable Tagged Neutron Inspection System (RRTNIS) shown in Figure 25, in which the neutron generator, the radiation shield, the detectors and the electronics are placed inside a container for outdoor operation, with temperature stabilization inside [60]. 

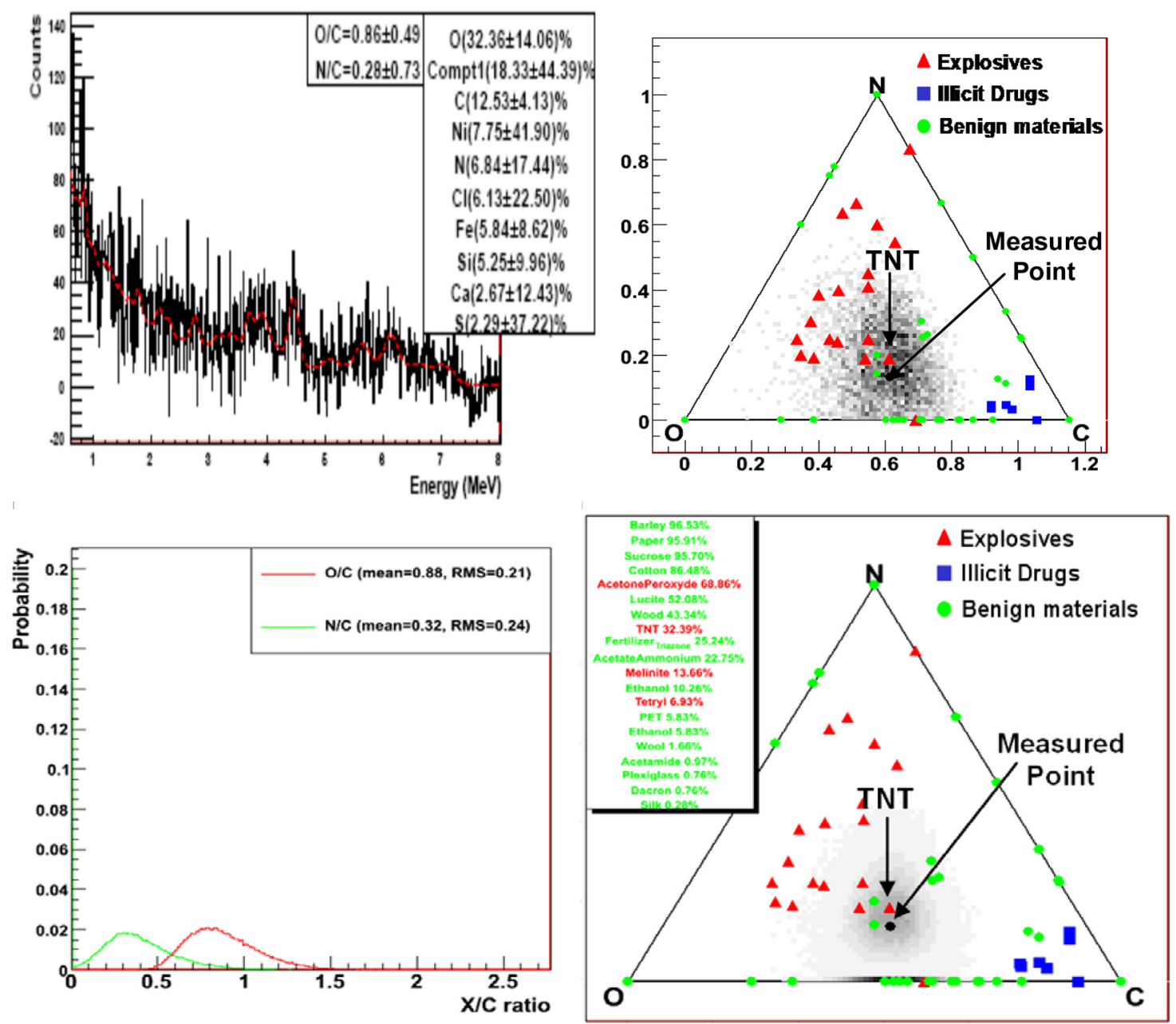

Fig. 23. Simulated inspection data with 32 top detectors for the TNT block in position 8 (see Fig. 19) of the wood cargo container. The gamma-ray spectrum (top, left) corresponds to the TNT area as shown in Figure 22. The first triangle diagram (top, right) shows the large uncertainty area due to statistical fluctuations and the next triangle diagram (bottom, right) shows the improvement with the synthetic spectra approach. The distributions of the $\mathrm{O} / \mathrm{C}$ and $\mathrm{N} / \mathrm{C}$ elemental ratios obtained with the synthetic spectra method are shown in the bottom, left panel.
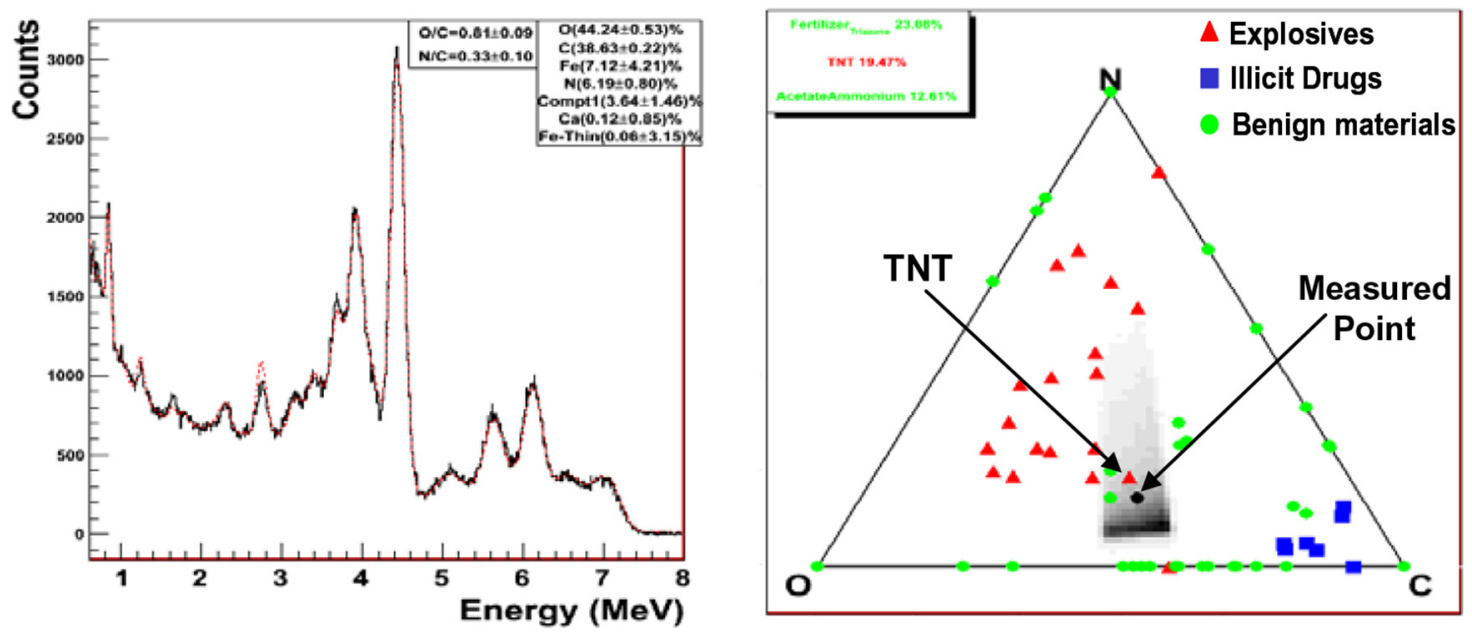

Fig. 24. Gamma-ray spectrum (left) and corresponding triangle plot (right) of a TNT target hidden in position 8 of a wood matrix acquired with the 10 reflexion detectors of a future industrial system. 

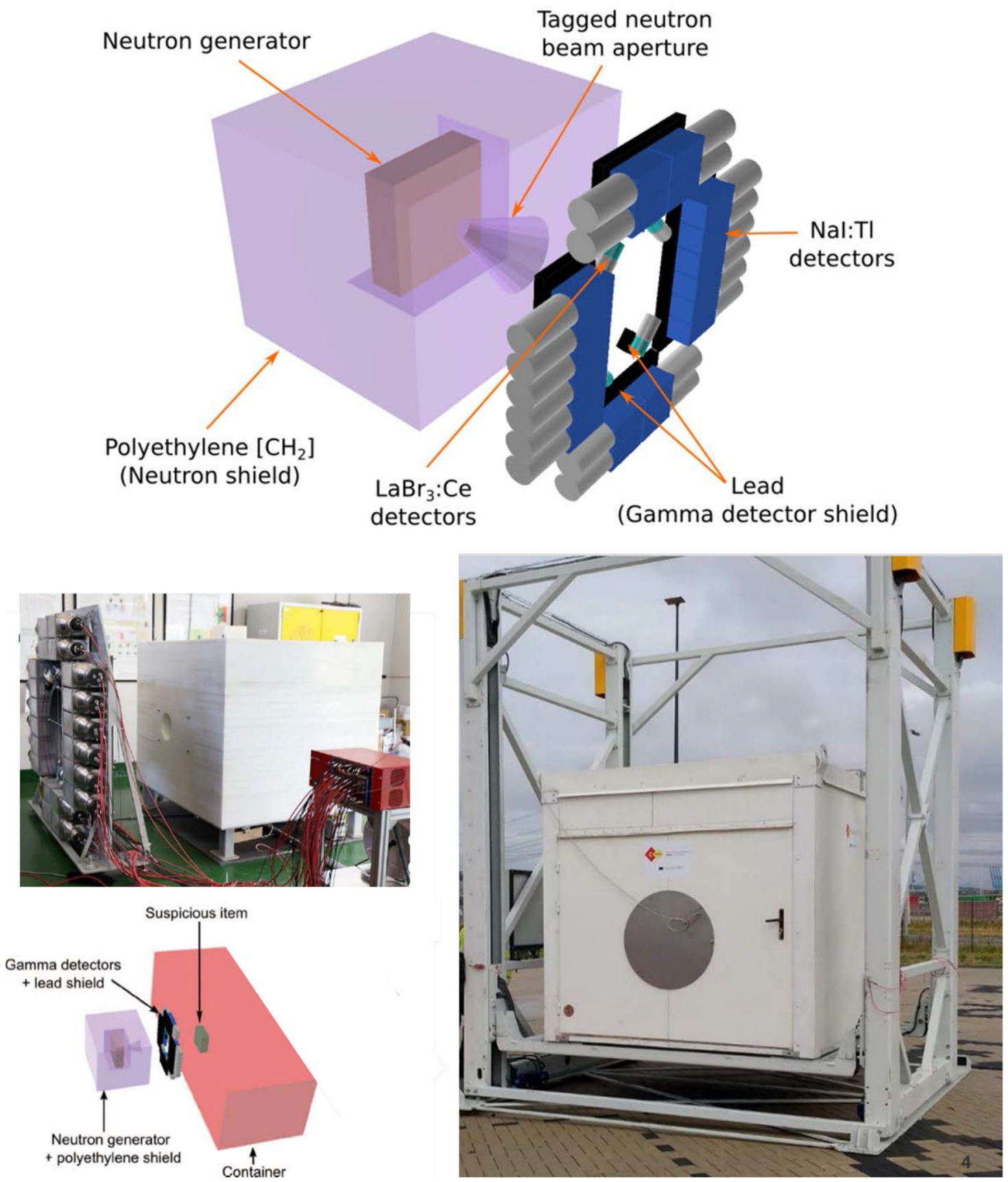

Fig. 25. C-BORD Rapidly Relocatable Tagged Neutron Inspection System sketches and pictures. The last picture (bottom, right) show the RRTNIS fully integrated inside a container mounted on an elevator frame.

Compared to the orthogonal position of the EURITRACK detectors relative to the neutron beam (see top detectors in Fig. 1), the backward C-BORD geometry advantageously uses cross sections angular dependency, which, as shown in Figure 10 for the case of carbon, often show an enhancement in the backward direction. It also allowed a very compact design, without detectors on the opposite side or above the container.

Being designed to allow mechanical simplification and system transportability, the RRTNIS also aimed at reducing the radiation safety area and thus the global system footprint. After dedicated Monte Carlo simulations, the $40 \mathrm{~cm}$-thick polyethylene cube shown in Figure 25 was designed, with a cone-shaped aperture for the tagged neutron beam. As shown in Figure 26, the radiation safety area for which the dose is lower than $1 \mu \mathrm{Sv} / \mathrm{h}$ (limit for nonradiation workers) corresponds to a $14 \mathrm{~m}$ side square when a beam dump is used, that is, a $50 \mathrm{~cm}$ thick polyethylene wall ( $4 \mathrm{~m}$ wide, $6 \mathrm{~m}$ high), to stop the neutron beamtransmitted beyond the container. This area is prolonged by about $28 \mathrm{~m}$ (total $42 \mathrm{~m}$ ) in the direction of the tagged neutron beam without beam-dump. The dose delivered to 
(a) With beam-dump

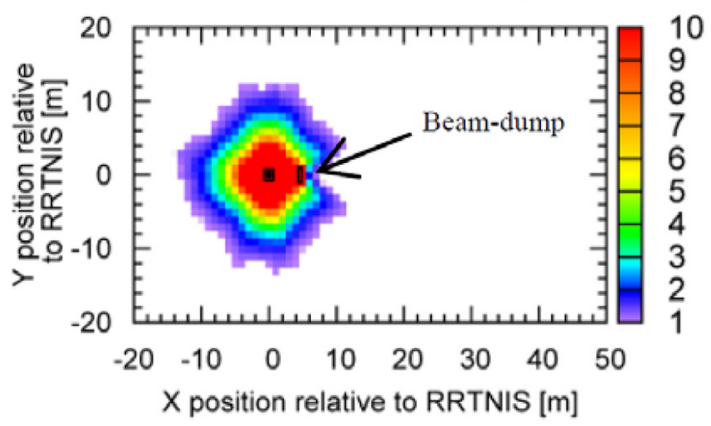

(b) Without beam-dump

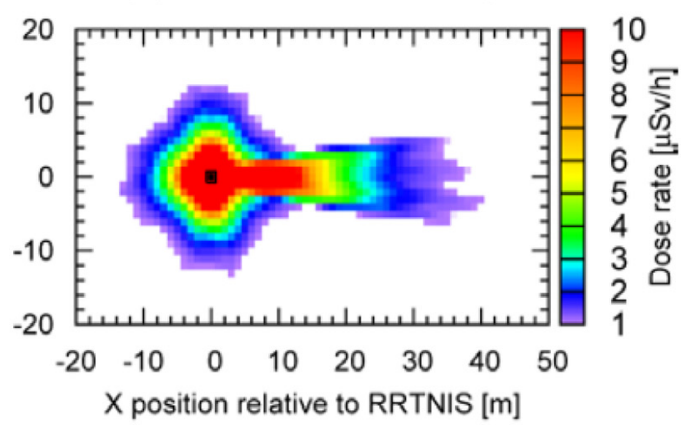

Fig. 26. Calculated radiation safety area associated to the RRTNIS with the adopted polyethylene shielding, using a beam-dump (a) and without beam-dump (b). The calculations correspond to $10^{8} \mathrm{n} / \mathrm{s}$ total emission.

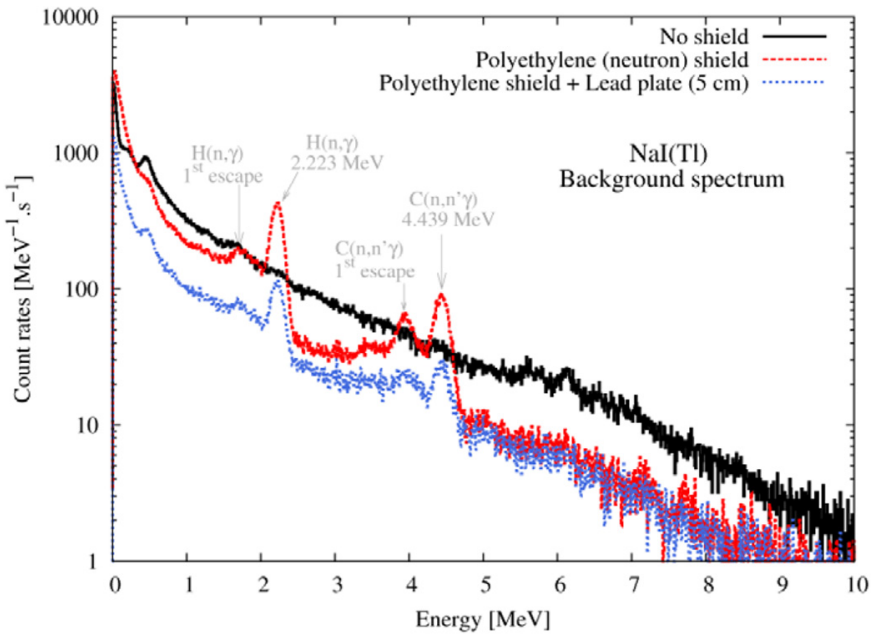

Fig. 27. MCNP6 simulation showing the random background brought by the polyethylene shield, with the effect of a $5 \mathrm{~cm}$ thick lead plate between detectors and polyethylene [60].

any stowaways present in the container would be extremely low because the dose rate is $1.2 \mathrm{mSv} \cdot \mathrm{h}^{-1}$ at a distance of $1 \mathrm{~m}$ of the bare neutron generator for its maximum emission of $10^{8} \mathrm{~s}^{-1}$, without any biological shielding. Taking into account the distance of almost $2 \mathrm{~m}$ between the neutron source (tritium target inside the generator) and the container wall, the nominal neutron emission of $510^{7}$ $\mathrm{s}^{-1}$ during $10 \mathrm{~min}$ neutron inspections, the neutron dose delivered to stowaways would be far less than $100 \mu \mathrm{Sv}$ (the neutron-induced gamma dose rate being one order of magnitude lower). In any case, the concept for use of the neutron inspection requires a prior radiography of the container by an X-ray scanner, which would enable to detect stowaways with a similar low delivered dose.

Although polyethylene is effective in reducing the radiation safety area, it can significantly increase the amount of background events in the scintillation detectors. Indeed, neutrons scattering on carbon and radiation capture by hydrogen nuclei can lead to 4.439 and 2.223 $\mathrm{MeV}$ gamma rays, respectively, which can significantly contribute to the total count rate. As shown in Figure 27, a $5 \mathrm{~cm}$ thick lead shield placed between detectors and polyethylene allows decreasing significantly the contribution of these two gamma rays in the gamma background spectrum, and consequently in the random background of the TOF spectrum (see in Fig. 5 for instance). Since the presence of a container also increases the detectors background due to backscattered neutrons and gamma rays, MCNP calculations have been performed to assess the total count rate taking into account both the polyethylene shield and the presence of a cargo container. In the worst case, consisting of a cargo container loaded with an iron cargo and a total neutron flux of $10^{8} \mathrm{n} / \mathrm{s}$, the count rate of the twenty $5^{\prime \prime} \times 5^{\prime \prime} \times 10^{\prime \prime} \mathrm{NaI}(\mathrm{Tl})$ scintillation detectors with a $600 \mathrm{keV}$ low-energy threshold is predicted to be $6.7 \times 10^{5} \mathrm{~s}^{-1}[60]$. With the same neutron flux, the total count rate of the four $3^{\prime \prime} \times 3^{\prime \prime} \mathrm{LaBr}_{3}$ scintillation detectors with a $100 \mathrm{keV}$ threshold is expected to be about to be about $4.3 \times 10^{4} \mathrm{~s}^{-1}$.

C-BORD is also an evolution of EURITRACK regarding the electronics. C-BORD data acquisition system is based on fast signal digitizers with an openaccess data acquisition software to handle CAEN electronics boards shown in Figure 28. Such a data acquisition results in more compact electronics and an enhancement of data processing capabilities [61-63].

Instead of discretizing the alpha detector area into pixels as in EURITRACK, the whole YAP detection area is used to calculate the barycentre of the scintillation light spot associated to each detected alpha particle. Such an approach offers more flexibility for selecting the tagged neutron beam aperture. The measured radial tagged neutron beam resolution is about $10 \mathrm{~cm}$, at $2 \mathrm{~m}$ distance from the neutron generator [64]. The typical alpha maps shown in Figure 29 evidences that the mapping between the true alpha position and the reconstructed alpha position is distorted due to incomplete light collection on the sides of the YAP detector, which will be corrected in future steps towards an industrial system. During C-BORD RRTNIS operation, only the central area indicated in black in Figure 29 was used.

New data processing and visualisation approaches have also been developed [65]. To check consistency between measurements and the manifest of the cargo container (declaration of transported goods) and identify materials in 


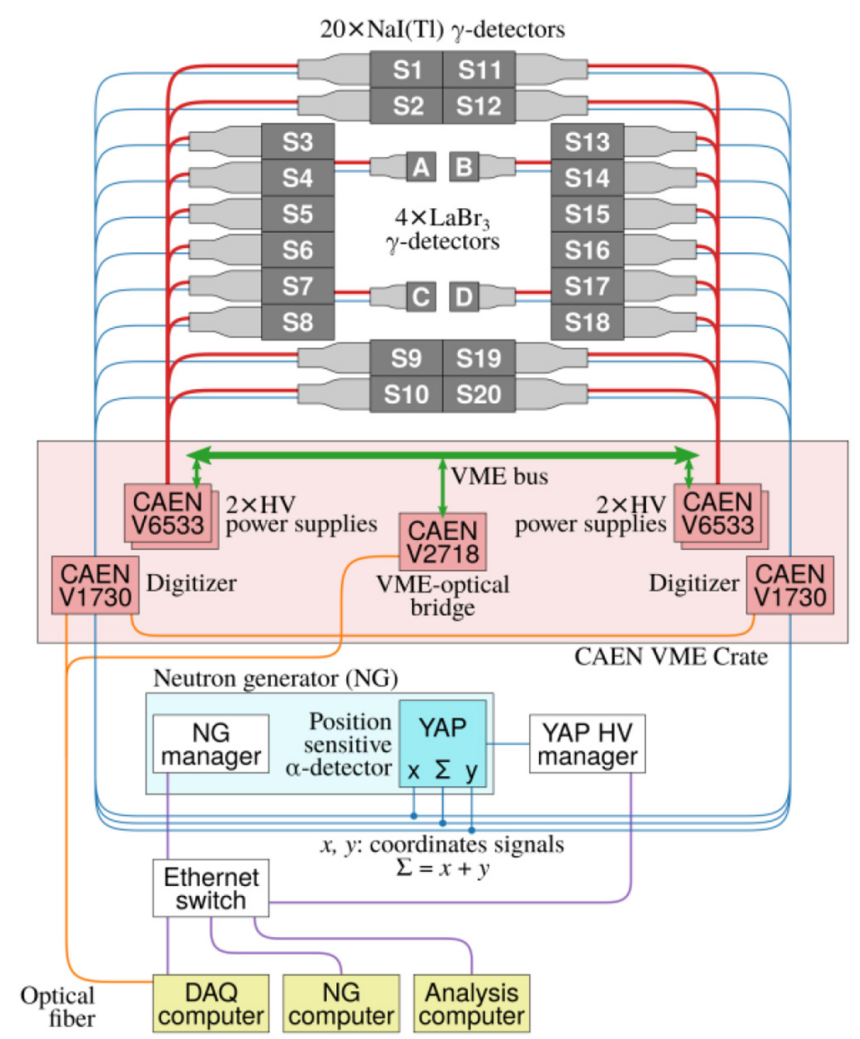

Fig. 28. Connections between the components of the CBORD RRTNIS data acquisition system.

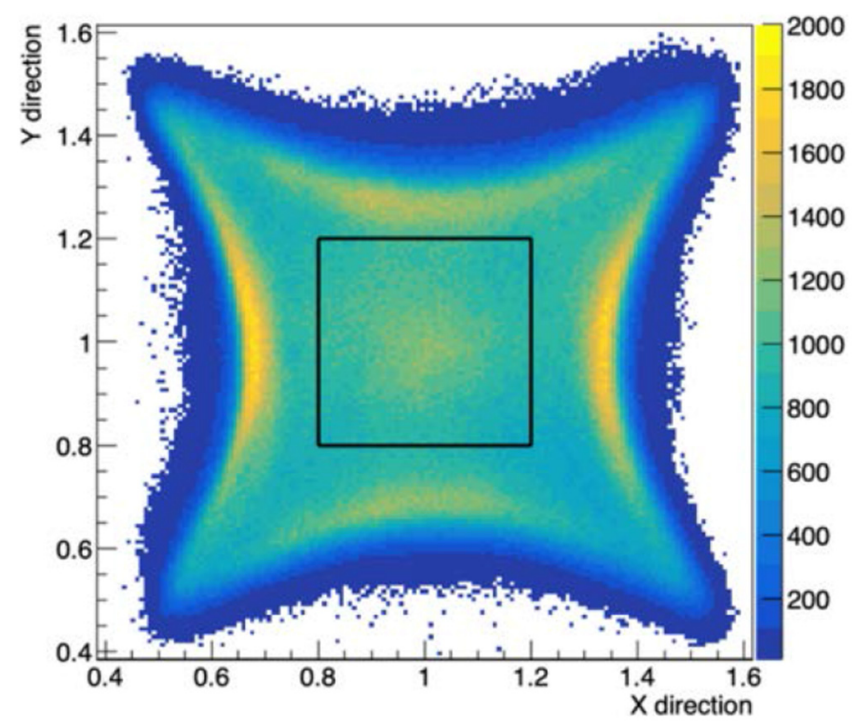

Fig. 29. Example of $(X ; Y)$ alpha map obtained after barycenter reconstruction of the alpha particle hit in the YAP detector.

a user-friendly way, the three spider charts shown in Figure 30 allow grasping easily their elemental composition and provides a rough classification. The first spider chart (c) provides elemental count fractions stemming from the tagged-neutron-induced gamma spectrum of the suspicious item, after unfolding with the database of elemental gamma spectra (similarly as in Fig. 3). The second spider chart (e) provides a first material classification within \{ceramic; organic; metal; neutron-induced noise; chemical\}, and for organic materials, the third spider chart(f) helps visualizing the probability of having explosives, illicit drugs or benign materials, according to the position of the unfolding result and uncertainties in the CNO triangle diagram (Fig. 3).

In addition, to have a better view of the in-depth composition of the cargo, the unfolding is performed along the beam axis with $2 \mathrm{~cm}$ steps as shown in Figure 31. For each slice, the material type is decomposed into its components among \{metal, ceramic, chemical, noise, explosive, benign, drug $\}$. Figure 31 shows the decomposition of a container loaded with an iron matrix inside which a pack containing $9 \mathrm{~kg}$ of $\mathrm{C} 4$ explosive simulant is hidden. The matrix is identified as a metal-like zone rich of iron in the material composition profile, whereas the $\mathrm{C} 4$ simulant is detected due to the presence of oxygen, nitrogen end carbon. The chemical component is due to the spurious presence of chemicals such as sulphur or phosphorus in small quantities when performing spectrum unfolding. Given that small components, with a contribution of a few $\%$, are likely statistical artefacts from the unfolding algorithm, such contributions are not considered as real. Further investigations are needed to improve the rejection and detectability of elements with small contributions in the unfolding.

Figure 32 shows an example of 10 min measurements with a neutron flux of $5 \cdot 10^{7} \mathrm{n} / \mathrm{s}$ [65]. The container is loaded with wood and a $10 \mathrm{~kg}$ RDX explosive simulant is hidden at a depth of $30 \mathrm{~cm}$ inside the wood matrix. The container iron wall is distinguishable as a metal-like zone at the beginning of the TOF whereas the explosive is distinguishable from the wood matrix due to its different carbon, oxygen and nitrogen composition. The second explosive peak at $290 \mathrm{~cm}$ is an artefact caused by statistical uncertainties, since the neutron beam intensity rapidly decreases with the distance inside the container wood matrix.

Experimental laboratory and field tests of C-BORD RRTNIS $[65,66]$ have demonstrated its capability to detect less than $20 \mathrm{~kg}$ of explosives or narcotics in iron or wood matrices having a density of $0.2 \mathrm{~g} \cdot \mathrm{cm}^{-3}$. These performances are a real step forward compared to EURITRACK system designed to detect $100 \mathrm{~kg}$ of TNT in the middle of a $0.2 \mathrm{~g} . \mathrm{cm}^{-3}$ iron cargo. Further improvements of data processing are now foreseen in the frame of a new H2020 project called ENTRANCE, which will start in October 2020. In particular, artificial intelligence with fuzzy logic trees will be used to improve material classification [67]. In addition, recent studies showed that, although no hydrogen peak can be measured using tagged neutrons, the use of artificial neural networks allows assessing hydrogen concentration using the APT, thus improving greatly the capability to identify organic materials [68].

\section{Special nuclear material detection with tagged neutrons}

\subsection{Introduction}

The risk of a terrorist attack using special nuclear materials (SNM) is seriously considered by the information agencies [69]. 
(a) Neutron flight path on Nal detectors

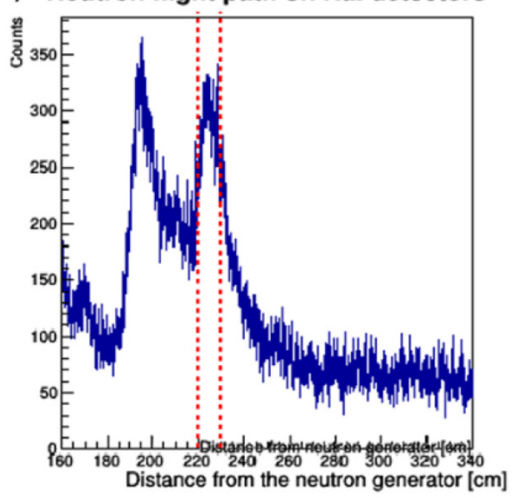

(b) Energy spectrum after spatial selection

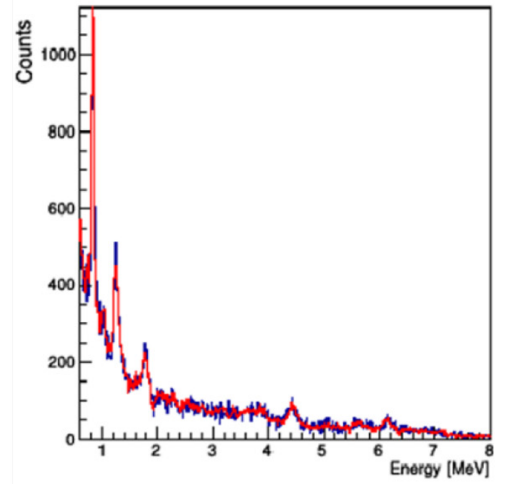

(c) Elemental count distribution

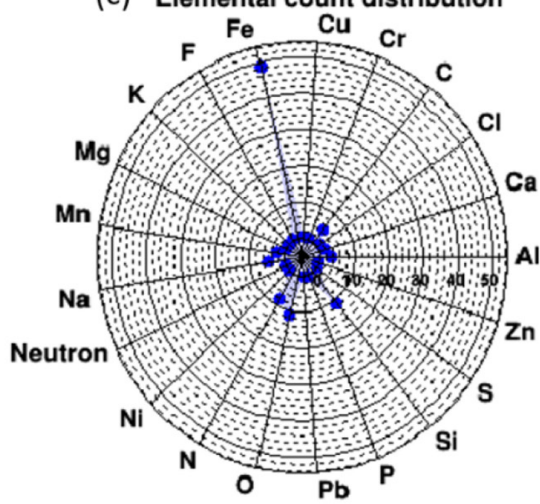

(d) Organic material identification

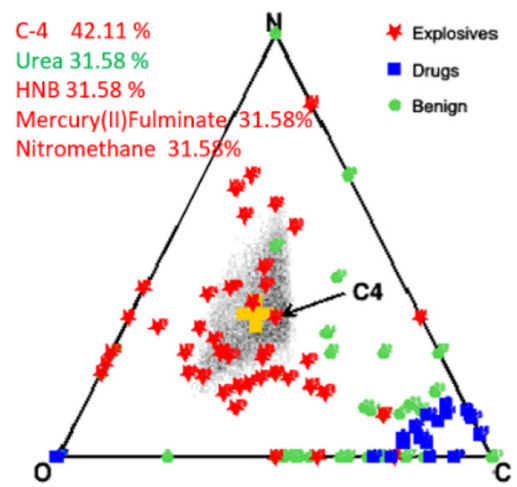

(e) Material classification

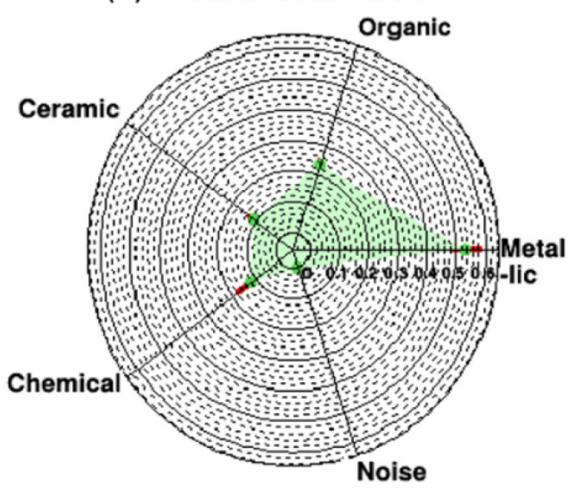

(f) Organic material classification

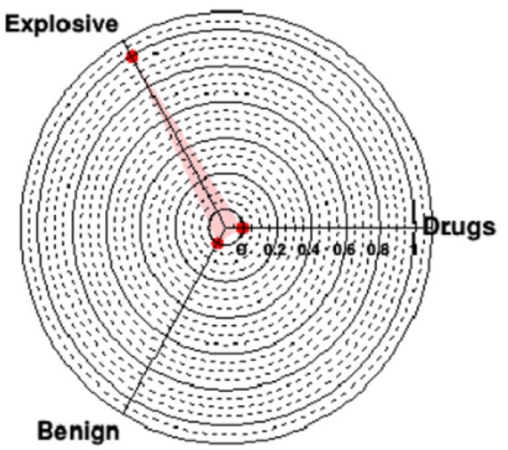

Fig. 30. Measurement of a $9 \mathrm{~kg} \mathrm{C} 4$ simulant target dissimulated at $26 \mathrm{~cm}$ in depth in an iron-filled container. Panel (b) displays the gamma spectrum for materials located at a distance between 220 and $230 \mathrm{~cm}$ from the neutron generator, which is the position of the C4 target (the peak at $200 \mathrm{~cm}$ corresponds to the container wall)selected in red dashed lines on panel (a). The "Elemental count fractions" displayed in panel (c) are coming from the unfolding procedure, which leads to the fit curve in red in panel (b).

Terrorists may try to acquire fissile material by purchase, diversion, or force, for the purpose of fabricating a crude nuclear bomb, known more formally as an "improvised nuclear device" (IND) [69,70]. Two types of fissile material could be used for this purpose, highly enriched uranium (HEU) or plutonium, but the former would be far easier to make into a successful IND. These materials have been produced in great quantity in nuclear weapon and civilian nuclear energy programs around the world. According to the conservative figures used by the International Atomic Energy Agency, only $25 \mathrm{~kg}$ of highly enriched uranium (HEU) or $8 \mathrm{~kg}$ of plutonium would be needed to manufacture a weapon. Over the past several decades, illicit nuclear material trafficking has significantly increased and with it the risk of misuse by terrorist groups [71-73]. Although in each case the amount of fissile materials remained insufficient to produce a nuclear device, they could be used with an aim of testing the detection means. Nuclear materials are detectable by conventional passive nuclear measurements provided that there is no shielding to hide their natural neutron and gamma radiations. In case of an efficient shielding, active systems are needed to induce detectable radiations.

Among non-destructive interrogation technologies to detect SNM, only fast neutrons or high-energy X rays produced by an electron LINAC are sufficiently penetrat- ing to perform in-depth inspection of cargo containers and to induce a clear fission signal $[22,74]$. In this section, we describe a method consisting in detecting fission prompt neutrons and gamma rays induced by $14 \mathrm{MeV}$ tagged neutrons, in coincidence with the alpha particle, to evidence SNM in cargo containers. When a 1st level inspection such as an X-ray scanner identifies a suspicious region of interest in the cargo container, that is, with a high apparent density and atomic number, the focused tagged neutron interrogation is used as a 2nd line nonintrusive technology to confirm the treat or clear the container.

As described above for non-nuclear cargo material identification, a position sensitive alpha detector embedded in a D-T neutron generator tags the $14 \mathrm{MeV}$ interrogating neutrons both in direction and emission time. However, contrary to non-nuclear elements (like C, $\mathrm{N}, \mathrm{O}, \mathrm{Fe}, \mathrm{Si}$, etc.), the fission prompt gamma spectrum of SNM does not show characteristic gamma peaks, but a continuum instead [75]. Therefore, as gamma spectroscopy is not applicable, coincidences between tagged-neutroninduced prompt fission particles are used to detect SNM. The time-of-flight (TOF) triggered by the alpha detection allows differentiating fission gamma rays $\left(30 \mathrm{~cm} . \mathrm{ns}^{-1}\right.$ speed) and neutrons (less than $5 \mathrm{~cm} . n s^{-1}$ ). Figure 1 reports 


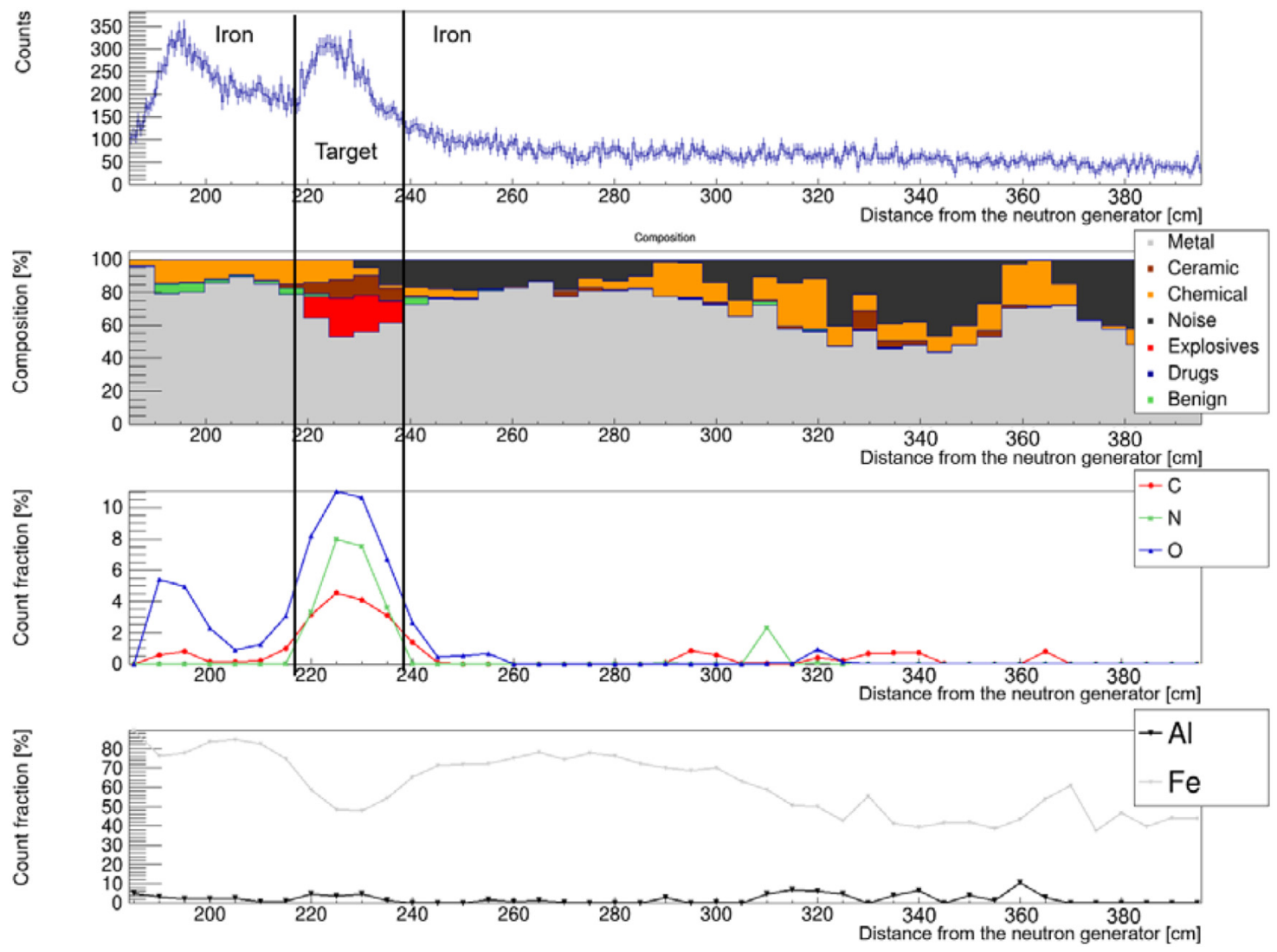

Fig. 31. Decomposition along the tagged beam direction of a container loaded with iron (density $0.2 \mathrm{~g} / \mathrm{cm}^{3}$ ), with $9 \mathrm{~kg}$ of $\mathrm{C} 4 \mathrm{simulant}$ being placed at a distance of about $20 \mathrm{~cm}$ from the container wall.

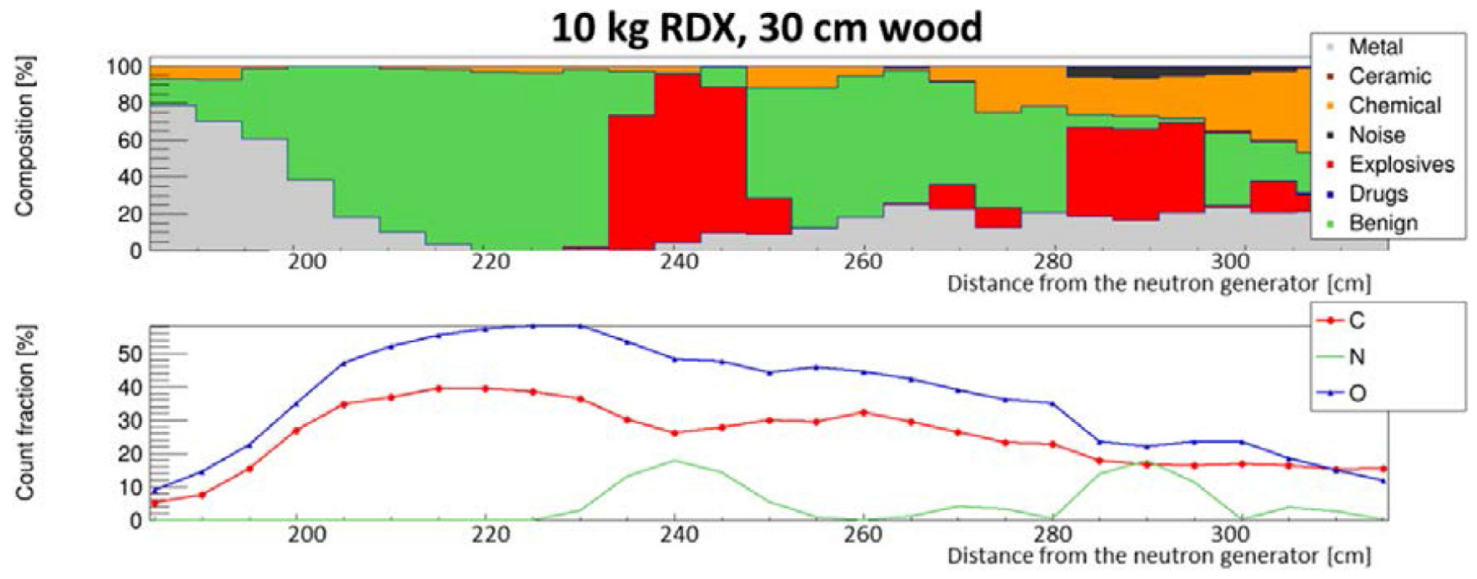

Fig. 32. Decomposition along the tagged beam direction of a container loaded with wood (density $0.2 \mathrm{~g} / \mathrm{cm}^{3}$ ), with $10 \mathrm{~kg}$ of RDX simulant being placed at a distance of about $30 \mathrm{~cm}$ from the container wall.

a schematic drawing of a tagged neutron inspection system for SNM detection in cargo containers. Coincidences between fission particles are detected in a large array of plastic scintillators (low-cost PVT) surrounding the container. A shield made of polyethylene and lead limits the count rate due to untagged neutrons, especially in detectors located near the neutron generator.

\subsection{Simulation}

The performances of the system are studied with MCNPPoliMi [76] Monte Carlo computer code, which provides information on individual histories (position of interactions, reaction types, time, energy, emission of correlated secondary particles...) allowing the simulation of such 

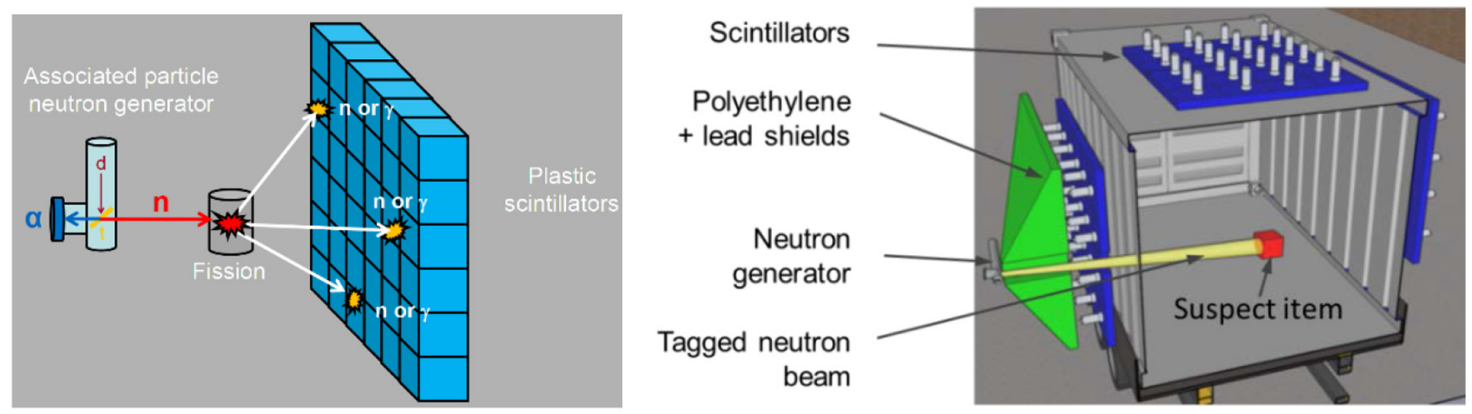

Fig. 33. Block diagram of the method and sketch of a cargo container tagged neutron inspection system with the beam focused on a suspicious item identified by an X-ray scanner.
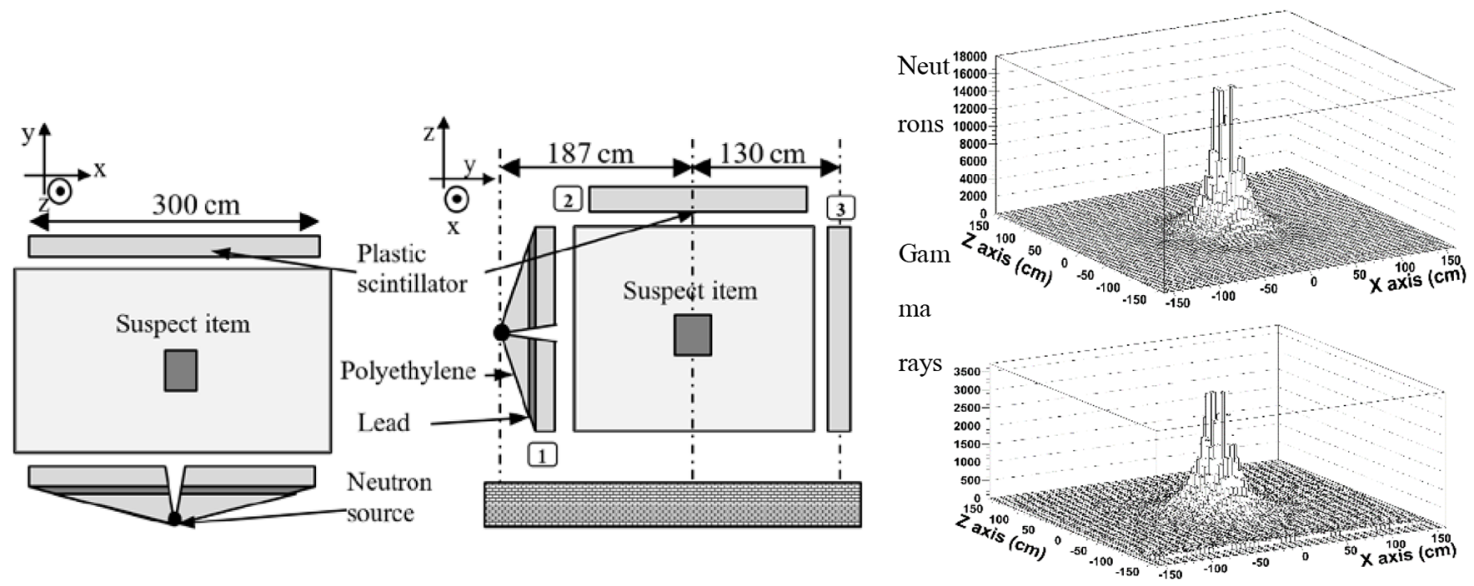

Fig. 34. Cross sections of the measurement system and simulation of the spatial distribution of neutron and gamma signals due to the neutron generator (isotropic $14 \mathrm{MeV}$ point neutron source) on detector panel 1 .

coincidence measurements. Output files are processed with dedicated software based on ROOT [77]. In addition to the useful signal induced by tagged neutrons, simulation also takes into account untagged neutrons emitted isotopically by the generator that create accidental coincidences and reduce the selectivity between nuclear and non-nuclear materials [78]. The simulation of an isotropic neutron emission with MCNP-PoliMi gives the main characteristics of random background events, that is, their neutron and gamma energy spectra, and their spatial distribution on detectors as illustrated in Figure 34. ROOT allows adding calculated real coincidences (induced by tagged neutrons) and random background events (due to untagged neutrons) by Monte Carlo sampling on their energy and spatial distributions [78].

The detection threshold is set at 0.1 MeVee ("ee" stands for "equivalent electron"), corresponding to about 0.67 $\mathrm{MeV}$ neutron energy (recoil proton on hydrogen nuclei in the plastic scintillators [79]). Inspection time is $10 \mathrm{~min}$ with a neutron generator total emission of $3.10^{7} \mathrm{~s}^{-1}$, from which less than $1 \%$ of tagged neutrons collides a small metallic target of about $2 \mathrm{~kg}$ HEU. Detector panels 1,2 and 3 of Figure 34 are made of $37.5 \mathrm{~cm} \times 40 \mathrm{~cm} \times 10 \mathrm{~cm}$ plastic scintillators, the size of which being a compromise between detection efficiency (three-fold coincidences statistics for an HEU cube in the center of the container), neutron and gamma scattering cross-talk between adjacent detectors, and cost (number of photomultipliers and electronic channels). A pyramid-shape polyethylene and lead plates protects detectors of panel 1 from direct irradiation by the $14 \mathrm{MeV}$ neutrons of the generator, as shown in Figures 33 and 34. Polyethylene slows down fast neutrons and lead stops gamma rays produced in polyethylene by radiative capture $(n, \gamma)$ and inelastic scattering $\left(n, n^{\prime} \gamma\right)$ reactions on hydrogen and carbon nuclei, respectively. Because $90 \%$ of the total count rate comes from detector panel 1 ( $7 \%$ in panel 2 and $3 \%$ in panel 3 ), these 45 and $5 \mathrm{~cm}$ thicknesses of polyethylene and lead, respectively, reduce the total count rate from $7.6 \times 10^{6}$ to $2.8 \times 10^{5} \mathrm{~s}^{-1}$, for a $3 \times 10^{7} \mathrm{~s}^{-1}$ total neutron emission.

\subsection{Coincidence analysis}

Figure 35 represents the histogram of time between the tagged neutron emission and the detection of induced coincidences including one, two, or three neutrons or gamma rays. The reported time-of-flight corresponds to the first-detected neutron or gamma ray. About $2 \mathrm{~kg}$ of smuggled metallic HEU is present in the center of the container filled with a $0.2 \mathrm{~g} . \mathrm{cm}^{-3}$ iron cargo. Containers 


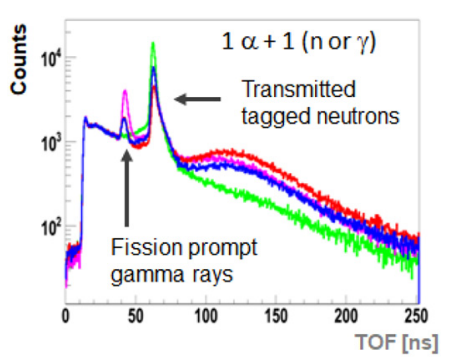

Unshielded HEU

HEU in $\mathrm{Cd}+\mathrm{Pb}$ shield

$\mathrm{Cd}+\mathrm{Pb}$ shield (without HEU)

Bare iron matrix
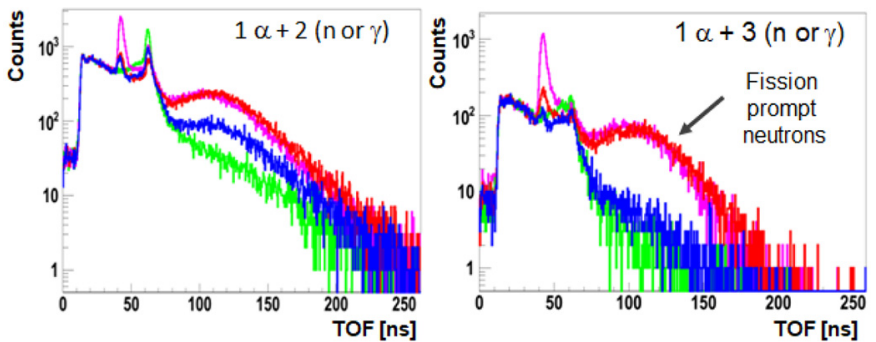

Fig. 35. Simulation of one-fold (above), two-fold and three-fold coincidences (below) after a 10 min inspection of a container filled with a $0.2 \mathrm{~g} . \mathrm{cm}^{-3}$ iron cargo. Neutron generator total emission is $3 \times 10^{7} \mathrm{~s}^{-1}$ and a HEU block, bare or in cadmium and lead shielding, is in the center of container. For comparison, simulations without nuclear materials (shield alone or bare iron matrix) are also reported.

transporting metallic goods represent about $15 \%$ of the maritime traffic and the average fill density of cargo containers is close to $0.2 \mathrm{~g} \cdot \mathrm{cm}^{-3}[57,58]$. HEU is here shielded by a $2 \mathrm{~mm}$ layer of cadmium to prevent from thermal neutron interrogation [31], and by $3 \mathrm{~cm}$ of lead to cut low-energy gamma and X-rays of ${ }^{235} \mathrm{U}$ (mainly below $205 \mathrm{keV}$ ) that could be detected by passive radiation portal monitors.

The first peak of the spectra, near $40 \mathrm{ns,} \mathrm{corresponds} \mathrm{to}$ induced-fission prompt gamma rays, and the second one, near $60 \mathrm{~ns}$, is due to tagged neutrons transmitted across the container that are detected in panel 3. In case of two- and three-fold coincidences, these last are detected with one or two uncorrelated particles, respectively.

The TOF window allowing the best discrimination between fission neutrons and benign material background is between 70 and $230 \mathrm{~ns}$. High multiplicity coincidences allows unambiguously evidencing SNM, as also shown in Figure 36. Indeed, as tagged neutrons may also interact by $(\mathrm{n}, 2 \mathrm{n})$ or $\left(\mathrm{n}, \mathrm{n}^{\prime} \gamma\right)$ reactions in the iron cargo and metallic shields surrounding SNM, two-fold coincidences are not sufficient and three-fold coincidences are used thereafter. Since tagged and fission prompt neutrons are not much attenuated by metallic shields, bare and shielded HEU multiplicity distributions are very close. Residual threefold coincidences in case of non-nuclear materials are due to cascade gamma rays following inelastic scattering (and other fast-neutron reactions generating gamma rays), in combination to scattering cross talk between adjacent detectors. Indeed, one particle can lead to multiple pulses after elastic neutron scattering or gamma Compton scattering in plastic scintillators. To mitigate cross talk,

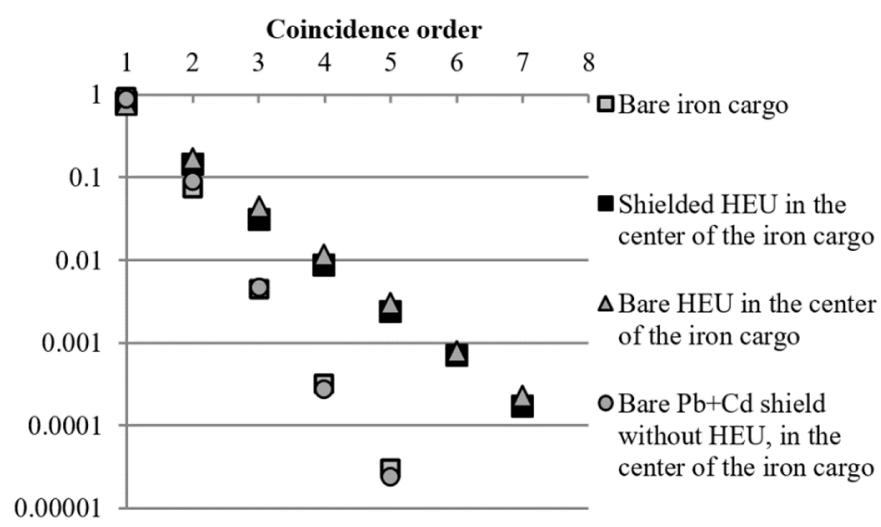

Fig. 36. Normalized distributions of coincidence orders in the (70 ns, $230 \mathrm{~ns})$ window.

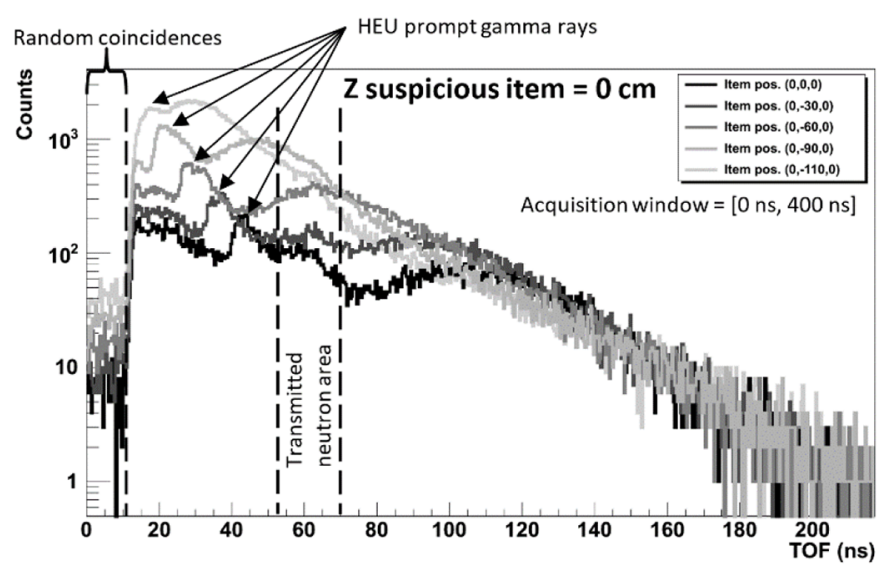

Fig. 37. Simulation of induced three-fold coincidences with HEU shielded by cadmium and lead at different depths inside the $0.2 \mathrm{~g}$. $\mathrm{cm}^{-3}$ iron cargo. The y position of HEU is the distance along the tagged neutron beam. The origin of the coordinate system $(0,0,0)$ is the center of the cargo container and $(0,-110,0)$ is the position (in cm units) close to the container wall, near the neutron generator and detector panel 1 (see Fig. 34).

we use large detectors $(37.5 \mathrm{~cm} \times 40 \mathrm{~cm} \times 10 \mathrm{~cm})$ and in addition, pulses recorded in neighbouring detectors are considered due to the same particle, with a time corresponding to the first detected particle [80]. However, cross-talk mitigation also leads to fission coincidence losses, especially when SNM is close to a detector panel, as reported further in Section 3.5, and a trade-off between is necessary.

\subsection{Data processing of cargo container inspections}

When the distance decreases between the suspicious item and detector panel 1 close to the neutron generator, the TOF difference between the prompt fission gamma peak and neutron "bump" reduces to reach a minimum of about 10 ns (see Fig. 37), which is approximately the 14 $\mathrm{MeV}$ tagged neutron TOF to reach the container wall [78]. In addition, the transmitted neutron peak overlaps 


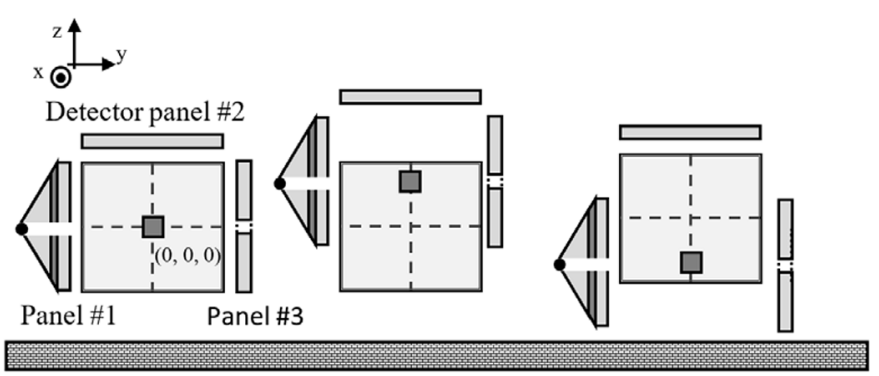

Fig. 38. Detectors and generator setups according to the suspicious item positions.

with the prompt fission neutron bump when the y position (depth inside the container, with the origin of the coordinate system in the center of the container) of the inspected object is between -110 (i.e. close to the container wall located near the neutron generator, see Fig. 34) and $-60 \mathrm{~cm}$. Therefore, a hole in the center of detector panel 3 is added in the simulation (see Fig. 38) to limit the contribution of transmitted tagged neutrons. This design is favorable to the implementation of a second neutron generator on the side of detector panel 3 to allow symmetric inspection on both sides of the container.

As the TOF of prompt fission neutrons strongly depends on SNM position along y-axis (depth inside the container), the time window of the neutron "bump" needs to be adapted accordingly. If a very dense suspicious item is identified with a first level X-ray scanner, its $\mathrm{x}$ and $\mathrm{z}$ positions on the $\mathrm{X}$-ray radiography allows adapting the system configuration as shown in Figure 38. The second configuration avoids saturation of detectors in panel 2 when the inspected item is on the top of the container, and improves the detection of fission particles in coincidence because a larger distance between SNM and panel 2 increases the probability that they reach different detectors. This adaptable setup also limits solid angle variations between detectors and SNM [78].

The y-position of a dense suspicious item (i.e. its depth inside the container) can be calculated using the TOF of the gamma-ray peak produced by tagged neutrons through inelastic scattering, fission, or other fast-neutron reactions. This peak is easy to detect in the one-fold coincidence spectrum, see Figure 35, or below in Figure 39 with a lead cube inside a $0.2 \mathrm{~g} . \mathrm{cm}^{-3}$ wood matrix.

The relationship between the gamma peak TOF and suspicious item y-position can be analytically calculated from the $14 \mathrm{MeV}$ neutron and gamma-ray speeds, that is, 5.13 and $30 \mathrm{~cm} \cdot \mathrm{ns}^{-1}$ respectively. This approach is consistent with Monte Carlo calculations, see Figure 40.

The borders of the neutron window are adjusted to the y-position of the suspicious item (depth inside the container) with a lower limit corresponding to the minimum detectable neutron energy of $0.67 \mathrm{MeV}(0.1$ MeVee electronics threshold), and an upper limit corresponding to a maximum energy of $9 \mathrm{MeV}$ that encompasses nearly all fission neutrons [78]. Figure 41 shows the limits of this time window, which are for instance 67 and $151 \mathrm{~ns}$ for a

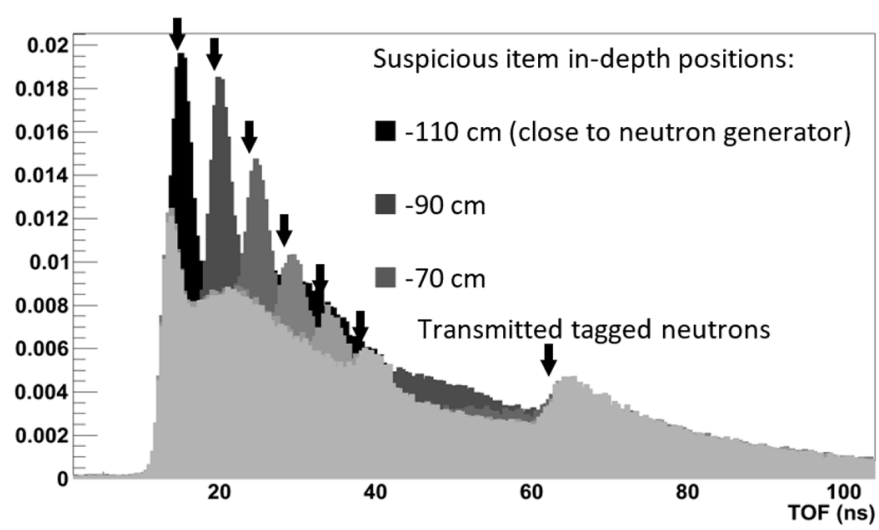

Fig. 39. Normalized one-fold coincidence spectrum of a lead cube at different depth inside a wood matrix, with arrows showing the inelastic scattering gamma peak produced in lead.

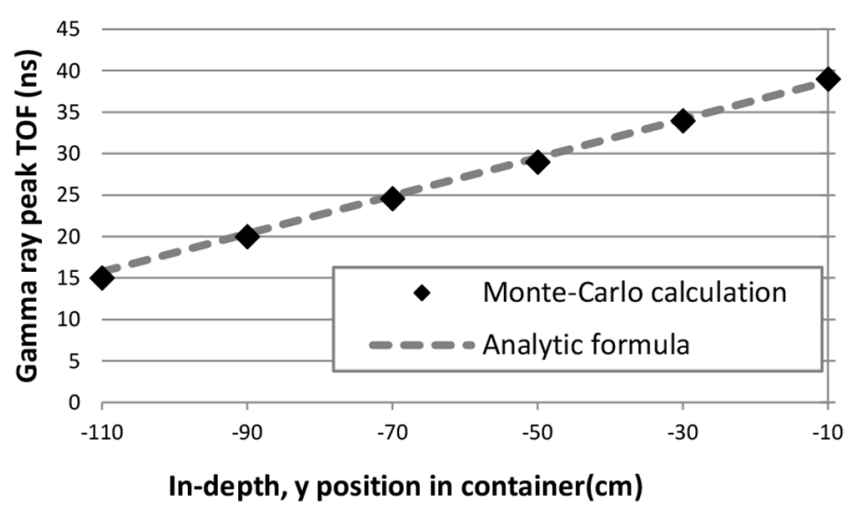

Fig. 40. TOF of the gamma peak of Figure 39 according to the suspicious item y position in the container.

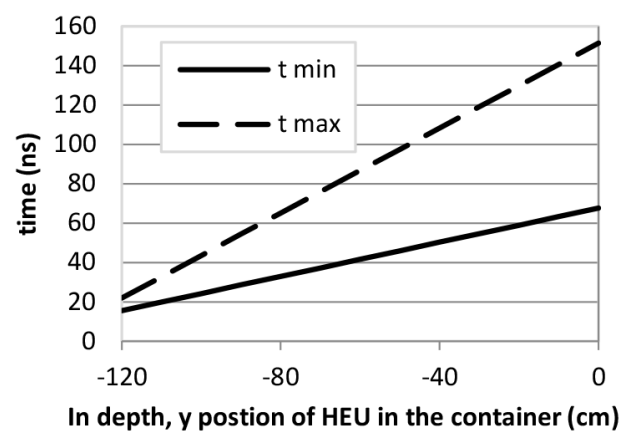

Fig. 41. Windows boundaries as a function of SNM depth (yposition) in the inspected container.

suspicious item in the center of the container $(\mathrm{y}=0 \mathrm{~cm})$, instead of 70 and $230 \mathrm{~ns}$ as initially proposed from Figure 35. The upper bound is significantly smaller to record as little as possible accidental coincidences and nonfission particles induced by tagged neutrons, see Figure 37. Nevertheless, almost all fission prompt neutrons are still detected, see Figure 35. 
The complete $(\mathrm{x}, \mathrm{y}, \mathrm{z})$ position of the suspicious item inside the container is valuable for end-users, and coincidence analysis provides additional information to make a decision, about possible SNM presence. To this aim, we compare three-fold coincidences recorded with a tagged neutron beam focused on the suspicious and with another beam aiming at a neighboring region of the cargo container, out of the suspicious item. This is done from the same measurement data set, by selecting different tagged neutron beams thanks to the position sensitive alpha detector. Three-fold coincidences recorded out of the suspicious item area (identified on the X-ray radiography) allows assessing the cargo material background, SNM detection being based on this ratio of three-fold coincidences as illustrated below.

\subsection{Simulated performances for cargo containers}

A large number of simulations have been performed for a series of dense suspicious items with $(y, z)$ coordinates uniformly distributed in 6 depths (y) and 5 heights $(\mathrm{z})$ inside the $0.2 \mathrm{~g} . \mathrm{cm}^{-3}$ iron cargo, with 20 and $65 \mathrm{~cm}$ steps, respectively. These dense items are either HEU shielded by cadmium and lead, or metallic blocks of the same size made of pure iron or lead. The $\mathrm{x}$ coordinate corresponds to lengthwise direction along the cargo container, that is, the road axis when loaded on a truck. The driver stops the truck when the suspicious item is in front of the tagged neutron system, according to the X-ray radiography.

The three-fold coincidence ratio between the dense item (shielded HEU, pure iron or lead blocks) and the bare iron cargo is plotted in Figure 42 as a function of $(\mathrm{y}, \mathrm{z})$ coordinates. Counterintuitively, it is smaller when HEU is close to detection panels 1 or 3 , that is, when y tends to -110 or $+110 \mathrm{~cm}$, because fission neutrons are geometrically less likely to be detected in different scintillators. In addition, to limit scattering cross talk, counts recorded in adjacent detectors are considered due to the same particle, which enhances this effect. Therefore, the cross-talk mitigation algorithm could be adapted to SNM position along $y$-axis to improve the coincidence ratio. Nevertheless, this last ratio is already larger with HEU than with bare iron or lead blocks for any $(\mathrm{y}, \mathrm{z})$ position. On the other hand, when iron or lead blocks are close to the neutron generator (when y tends to $-115 \mathrm{~cm}$ ), their coincidence ratio also dramatically reduces below unity. When moving away from the generator (when y increases), their ratio increases but without exceeding 1.5, while the SNM ratio is larger than 1.5 in any $(\mathrm{y}, \mathrm{z})$ position. Therefore, a threshold of 1.5 is set to trigger SNM suspicion. On the other hand, as neutron scattering in cargo materials spreads out the tagged beam, contrast between SNM and non-nuclear items reduces beyond the center of the container $(\mathrm{y}>0 \mathrm{~cm})$. Therefore, a second generator is needed on the opposite side of the container to perform a face-to-face inspection of both sides.

Similar calculations performed with a $0.2 \mathrm{~g} \cdot \mathrm{cm}^{-2} \mathrm{SiO}_{2}$ matrix corresponding to other non-hydrogenous cargo (ceramics, glass, fiberglass wool, etc.) show similar results. However, performances are very different with organic cargo, or any cargo containing hydrogen. Note that the main studied categories of cargo materials (metals, ceramics and organics) represent about $90 \%$ of the maritime traffic [57,58]. Anyway, in organic cargo like wood, fast-tagged neutrons induce fewer fissions in SNM than in free-of-hydrogen materials. For instance, a tagged neutron induces 0.08 and 0.3 fission in a bare HEU block located in the center of $0.2 \mathrm{~g} . \mathrm{cm}^{-3}$ iron and wood cargo, respectively, due to larger neutron scattering (see Fig. 43) and slowing-down (see Fig. 8) in wood [81].

In turn, prompt fission neutrons undergo scattering and slowing down on hydrogen nuclei in organic cargo, which significantly decreases detection efficiency in plastic scintillators due to the low-energy threshold of $0.67 \mathrm{MeV}$ $(0.1 \mathrm{MeVee})$. For instance, counting statistics are very poor in the fission neutron window when HEU is in the center of a container filled with a $0.2 \mathrm{~g} . \mathrm{cm}^{-3}$ wood cargo, as shown in Figure 44 (compared to Fig. 35 with the iron cargo). Concerning the other positions inside the wood cargo, Figure 45 shows that the three-fold coincidence ratio does not allow distinguishing HEU (even unshielded) from nonnuclear materials in the central region of the container, with a threshold set at 1.2. However, SNM detection is still possible up to a depth comprised between 40 and $60 \mathrm{~cm}$.

However, in order to improve performances for organic cargo, it is possible to use the fission prompt gamma-ray peak that is still present in the three-fold coincidence spectrum of Figure 44 for unshielded HEU. As above for fission neutrons, we select here a time window around the gamma peak (width of $10 \mathrm{~ns}$ and position determined from Fig. 39) due to fission, or to other reactions like inelastic scattering in nonnuclear materials, to calculate a ratio of three-fold coincidences when the tagged beam aims at the dense suspicious item, or the bare wood matrix. Again, this ratio is plotted as a function of $(\mathrm{y}, \mathrm{z})$ coordinates as shown in Figure 46.

The lead (and cadmium) shield enveloping HEU absorbs nearly $90 \%$ of induced-fission gamma rays, the average energy of which being about $0.9 \mathrm{MeV}$. Consequently, fission gamma rays alone cannot be used to detect shielded SNM. Concerning unshielded HEU, prompt fission gamma rays leads to a high three-fold coincidences ratio, but so does the dense iron cube because of gamma cascades following inelastic scattering reactions [82], preventing from an unambiguous detection of SNM. Therefore, we combine the fission neutron and fission gamma-ray coincidence ratios, as shown in Figure 47, which allows evidencing shielded HEU in almost $50 \%$ of the positions, and more than $80 \%$ for unshielded HEU, with a threshold set to unity for both ratios. False alarms for iron or lead cubes represents nearly $10 \%$ each.

Work is in progress to continue improving performances for organic cargo, for instance by correlating data obtained with two neutron generators inspecting symmetrically both sides of the cargo container (face-to-face), and with a higher tagged neutron emission. Another work under progress concerns interrogation with thermal neutrons between the pulses of a DT neutron generator [83], which is complementary to fast neutron interrogation with the APT for rich-in-hydrogen cargo. 


\section{Shielded HEU}
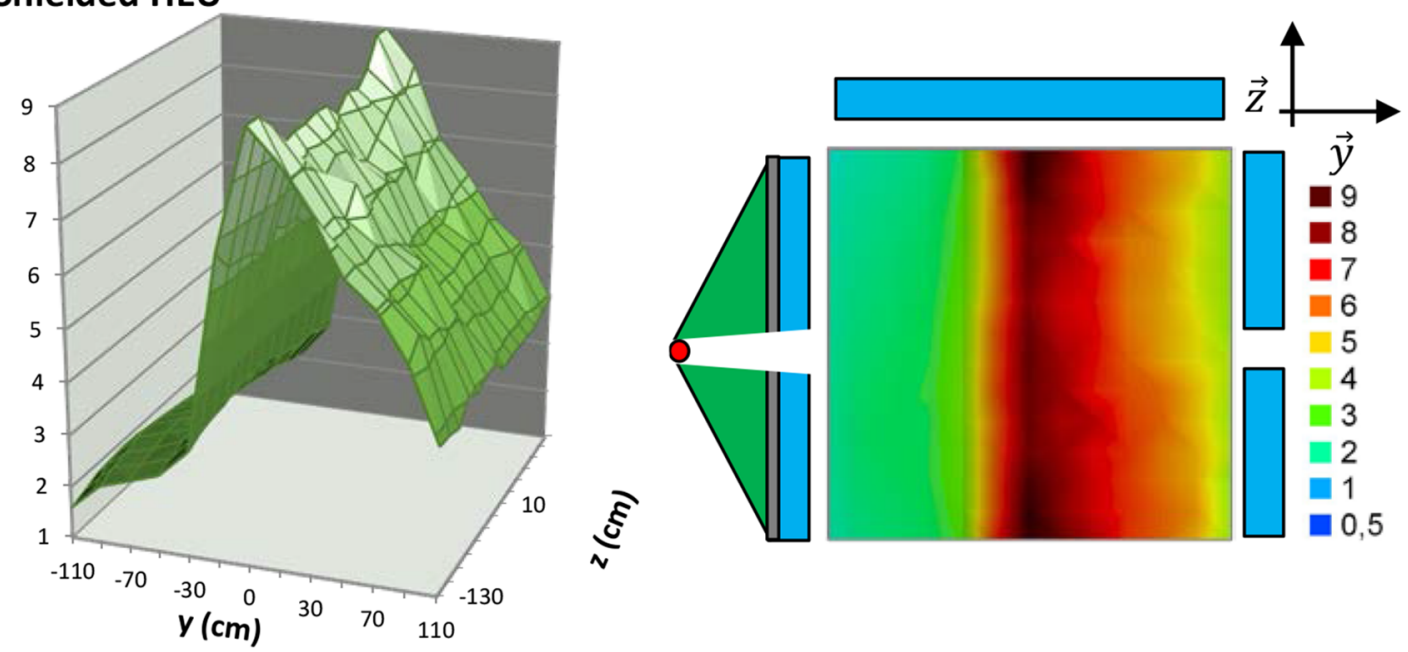

\section{Lead cube}
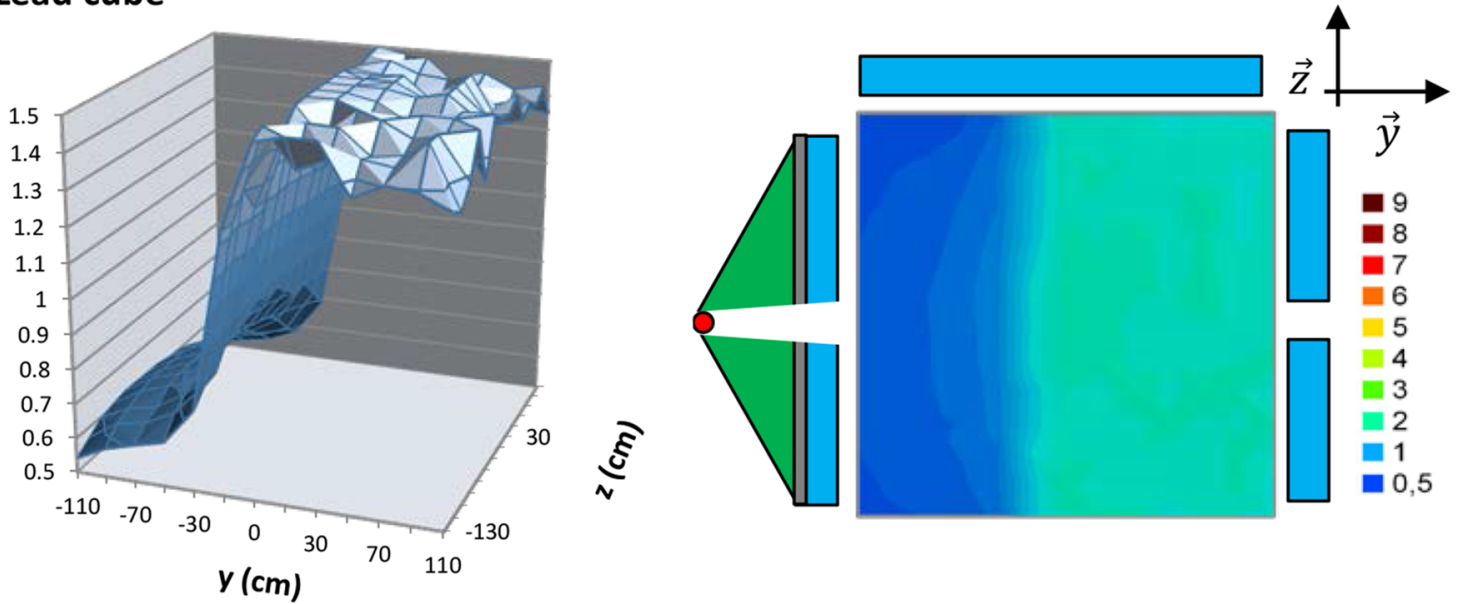

\section{Iron cube}
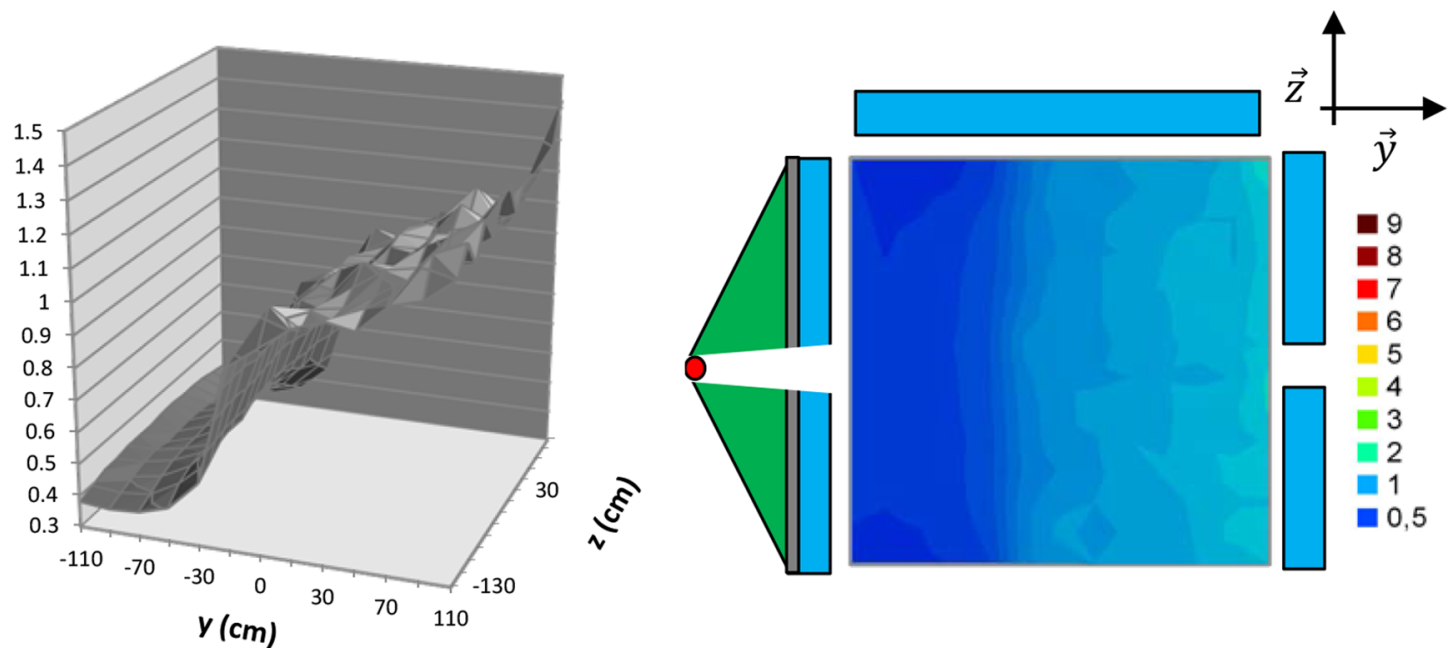

Fig. 42. Ratio of three-fold coincidences in the fission-neutron time window when the tagged neutron beam aims at the suspicious item (above: HEU shielded with $\mathrm{Cd}$ and $\mathrm{Pb}$; middle: bare iron block; below: bare lead block) to three-fold coincidences when the beam aims at another region of the $0.2 \mathrm{~g} . \mathrm{cm}^{-3}$ iron cargo. This ratio is given as a function of the $(\mathrm{y}, \mathrm{z})$ coordinates of the suspicious item in the container (left: $3 \mathrm{D}$ representation; right: $2 \mathrm{D}$ projection). The origin of the coordinate system is the center of the container: $\mathrm{y}=-110 \mathrm{~cm}$ corresponds to the first wall of the container (close to the neutron generator shown as a red point on the 2D projection maps) and $\mathrm{y}=+110 \mathrm{~cm}$ corresponds to the second wall. 


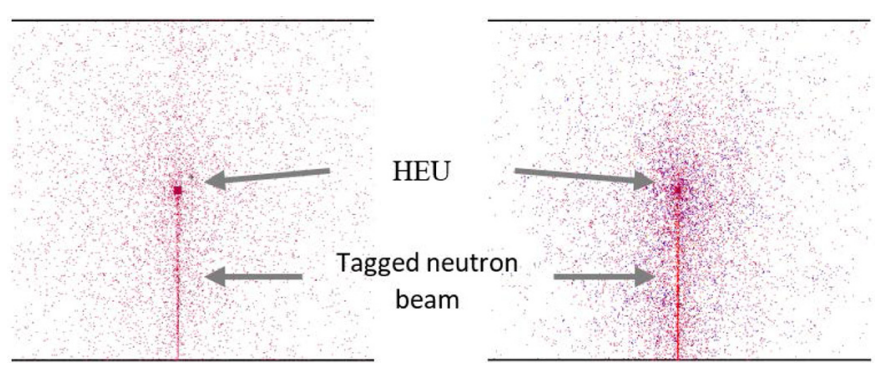

Fig. 43. Tagged neutron beam interactions in the 0.2 g.cm ${ }^{-3}$ iron (left) and wood (right) cargo, with an unshielded HEU block in the center.
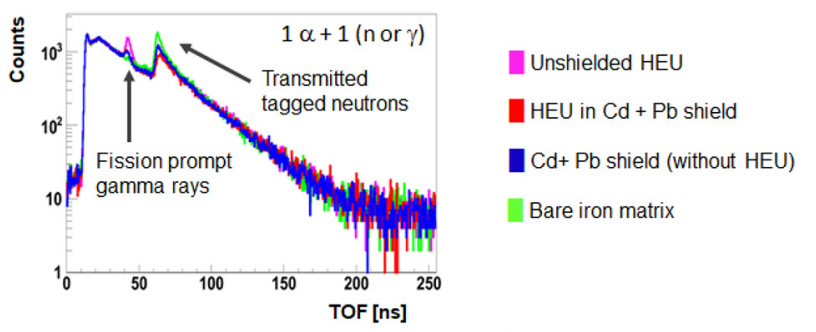

Bare iron matrix
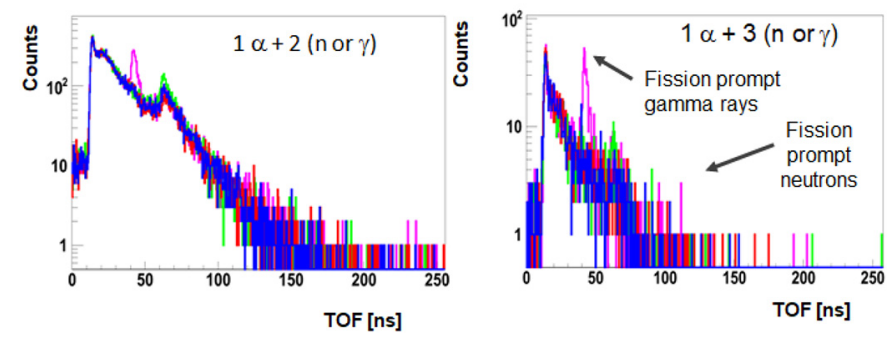

Fig. 44. Simulation of one-fold (above), two-fold and three-fold coincidences (below) after a 10 min inspection of a container filled with a $0.2 \mathrm{~g} \cdot \mathrm{cm}^{-3}$ wood cargo.

\subsection{Experimental validation of simulations}

To test the validity of simulations extensively used in previous sections, the experimental setup shown in Figure 48 is used. It reproduces at a small scale, with only height $10 \mathrm{~cm} \times 10 \mathrm{~cm} \times 100 \mathrm{~cm}$ plastic scintillators and a container mockup, the inspection of a cargo container [84] in which uranium, lead, and iron targets can be placed at different depths in $0.2 \mathrm{~g} . \mathrm{cm}^{-3}$ iron or wood matrices.

Figure 49 shows the active background measured without the container, in a $10 \mathrm{~min}$ acquisition with a total neutron generator emission of $2 \cdot 10^{7} \mathrm{n} / \mathrm{s}$. Despite poor counting statistics due to the limited number of detectors, the agreement between simulation and experiment is quite satisfactory. The TOF spectra show the interactions of tagged neutrons in the main parts of the setup, that is, the neutron generator envelop itself, and the wall of DANAIDES irradiation casemate (located behind the container mockup in Fig. 48).

The comparison between calculated and experimental TOF spectra with different targets of iron (Fig. 50), lead (Fig. 51) and uranium (Fig. 52) in the empty container shows that simulation correctly reproduces tagged neutron inspection (counting statistics, position and width of gamma peaks and neutron "bumps").
As the experimental setup only includes height $10 \mathrm{~cm}$ $\times 10 \mathrm{~cm} \times 100 \mathrm{~cm}$ plastic detectors, counting statistics is extremely poor for measurements with targets inside iron and wood matrices, especially for high multiplicity coincidences. Therefore, simulation was extensively used, as reported above, to estimate the performances of a fullscale inspection system with large plastic detection panels made of plastic scintillators around the maritime container (300 cm length along the container axis, $240 \mathrm{~cm}$ height of width, $10 \mathrm{~cm}$ thickness, as shown in Fig. 33).

\section{Conclusion}

Fast-tagged neutrons are an efficient probe to detect different types of threats or illicit goods (explosives, narcotics, nuclear materials) in maritime containers. The capability to identify the main elements constituting the cargo by neutron-induced gamma spectroscopy also allows checking consistency with the declaration of transported goods, in the fight against contraband. Tagged neutron inspection systems have been tested in real seaport environments, bringing valuable information in complement to 1st line X-ray scanners (less than 1-min inspections), to further analyse suspicious areas of the cargo container (in a typical 10-min 2nd line tagged neutron inspection) identified on the $\mathrm{X}$-ray radiography. Special Nuclear Material (SNM) detection is also possible by recording fission coincidences induced by tagged neutrons if a 1st line radiation portal monitors (RPM) triggers an alarm, and/or if the X-ray radiography reveals an unexpected dense object. Tagged neutrons would bring valuable information in complement to photofission, another 2nd line SNM detection technology mainly based on high-energy photons (interrogation with high-energy Xrays and detection of high-energy fission delayed gamma rays). Fast neutrons and high-energy photons having different penetration capabilities depending on cargo materials, especially a large attenuation in rich-in-hydrogen materials (like organic goods) for fast neutrons and in high-atomic number elements (like metals) for photons, coupling tagged neutron and photofission inspections would be complementary in view of SNM detection in a wide variety of cargo containers. A large platform combining non-intrusive inspection technologies (RPM, $\mathrm{X}$-ray scanners, neutron and photon inspection) was developed in C-BORD framework and will be optimized in another H2020 project called ENTRANCE, especially to include artificial intelligence in material recognition and decision making, as an assistance to end-users.

The Authors are extremely grateful to the many Colleagues and Partners who contributed to the technical achievements of this 15-year continuous effort work.

This research was funded by EURITRACK, FP6 Project, grant number 511 471, by Eritr@C, JLS Project, grant number JLS/2007/ISEC/550, by C-BORD H2020 project, grant number 653323, and by the CEA R\&D Program against CBRNE Threats. 

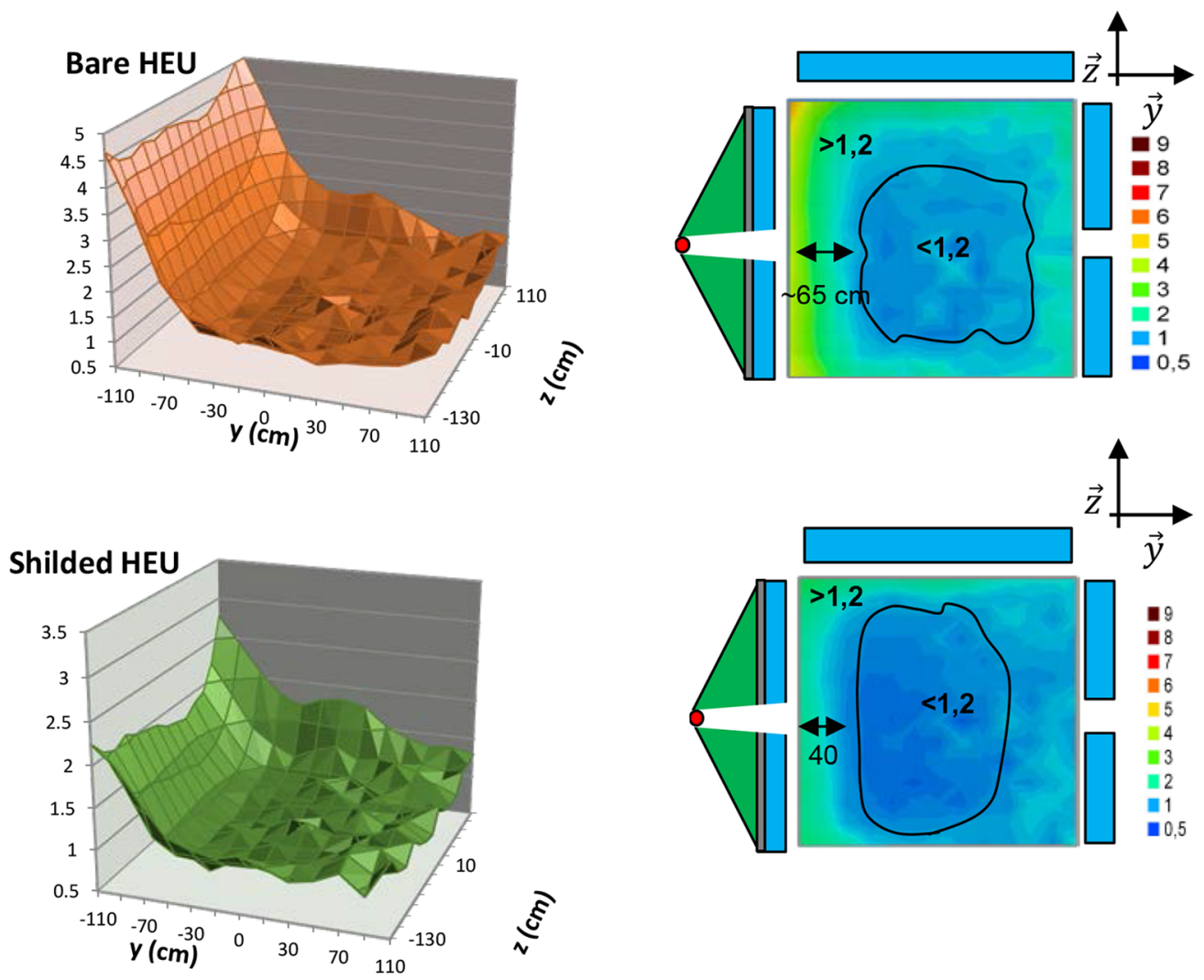

\section{Lead cube}
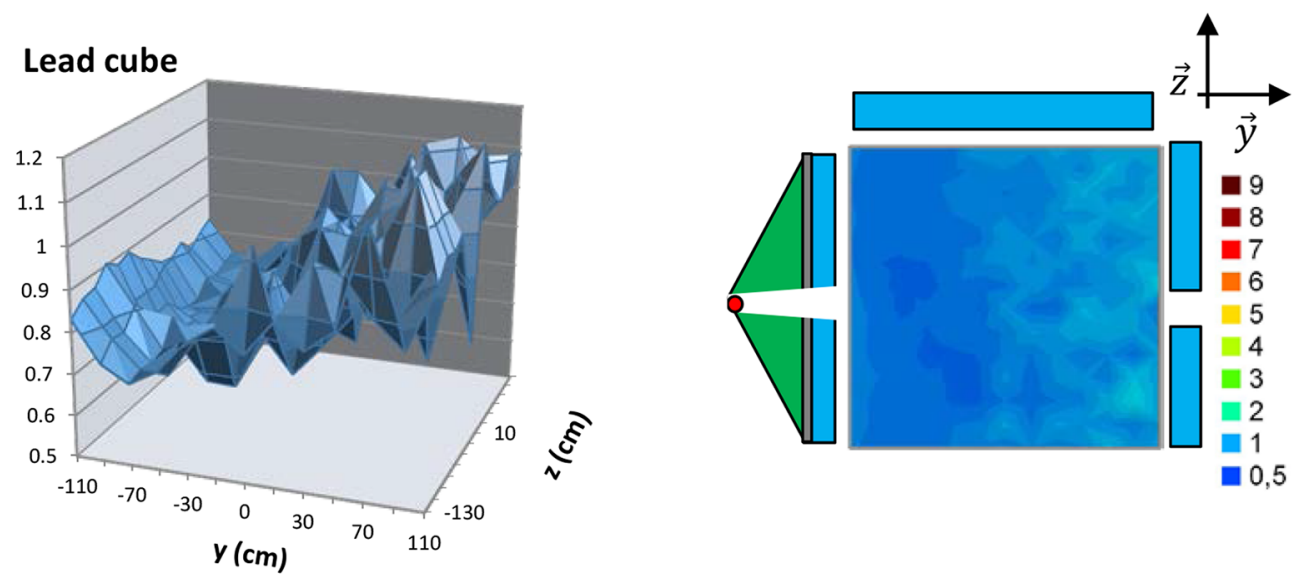

\section{Iron cube}
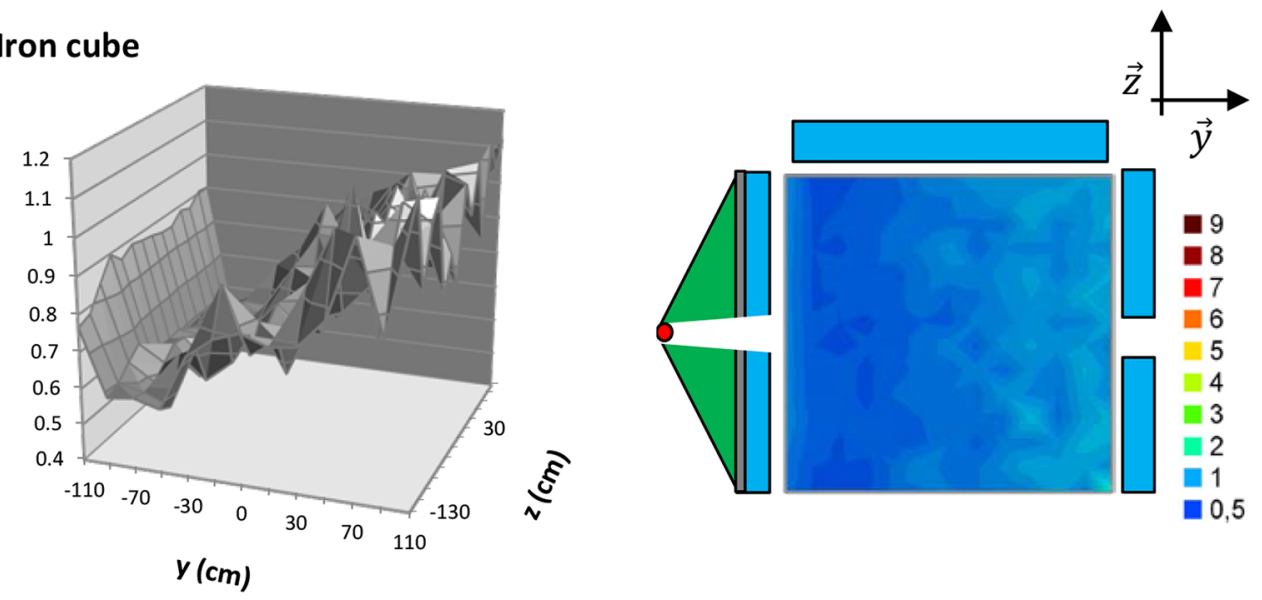

Fig. 45. Ratio of three-fold coincidences in the fission-neutron time window when the tagged neutron beam aims at the suspicious item or another region of the $0.2 \mathrm{~g} . \mathrm{cm}^{-3}$ wood cargo. 


\section{Shielded HEU}

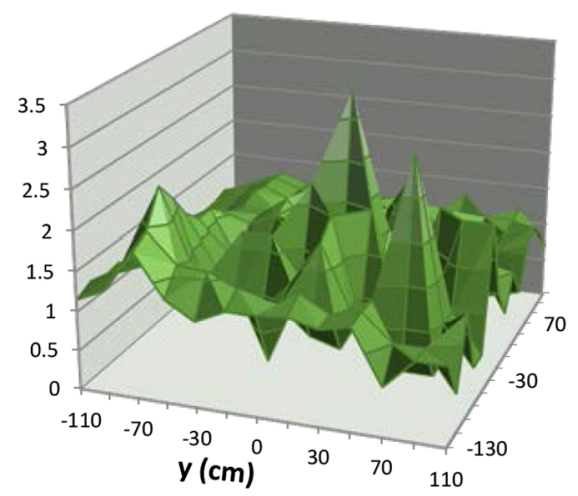

Bare HEU

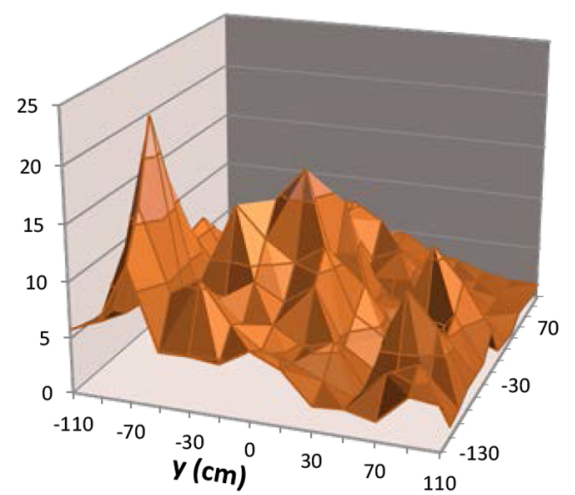

\section{Lead cube}

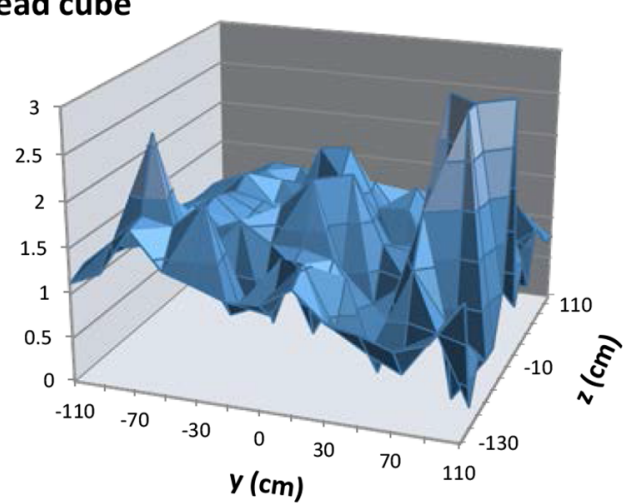

Iron cube

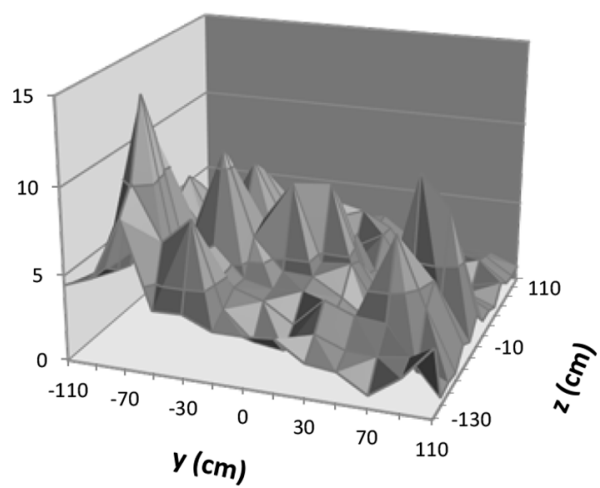

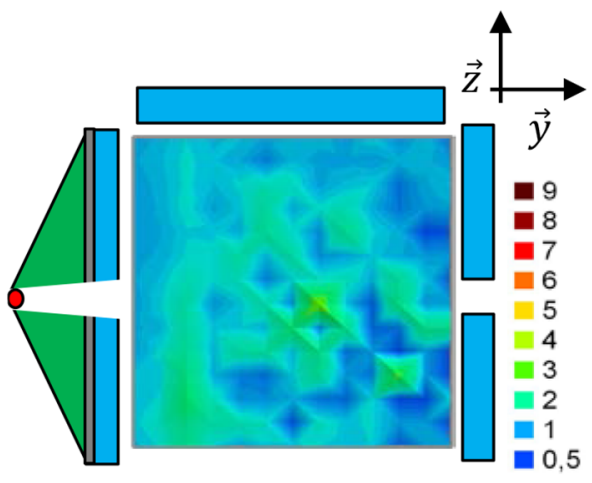
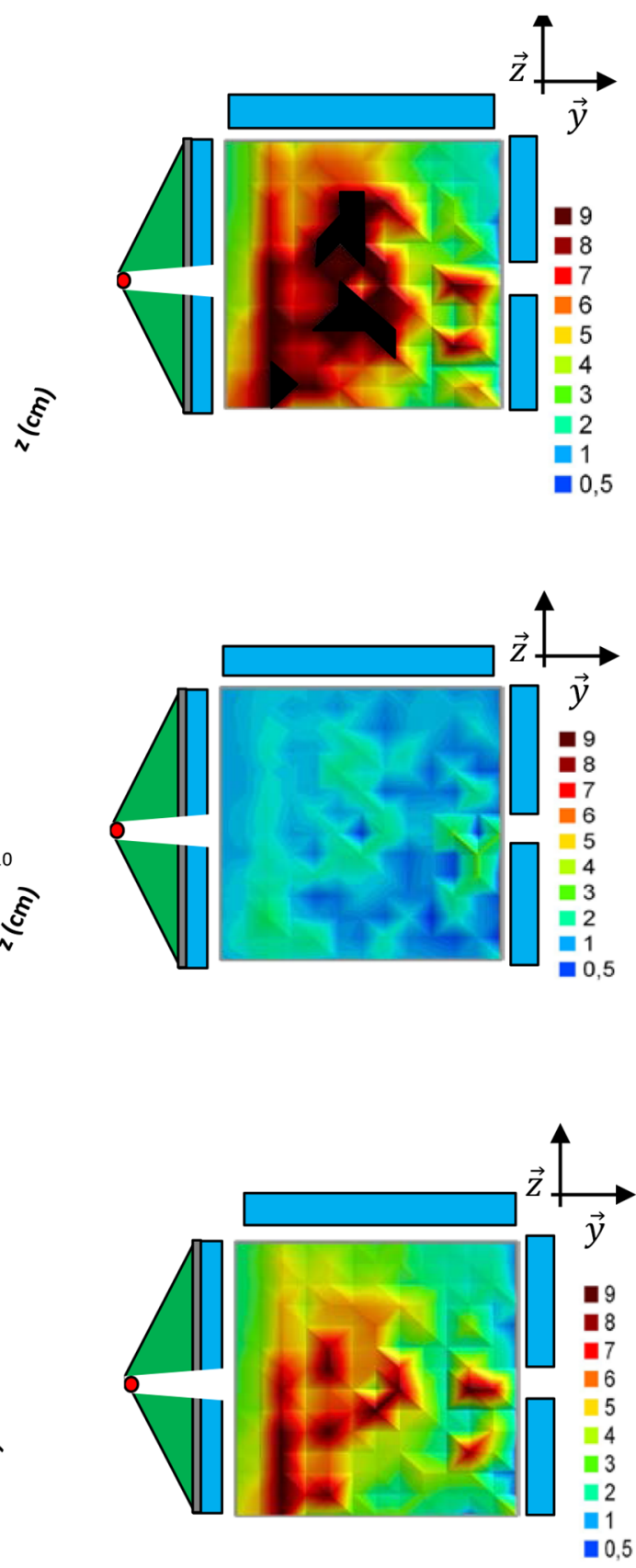

Fig. 46. Ratio of three-fold coincidences in the time window of fission prompt gamma rays when the tagged neutron beam aims at the suspicious item or another region of the $0.2 \mathrm{~g} . \mathrm{cm}^{-3}$ wood cargo. 


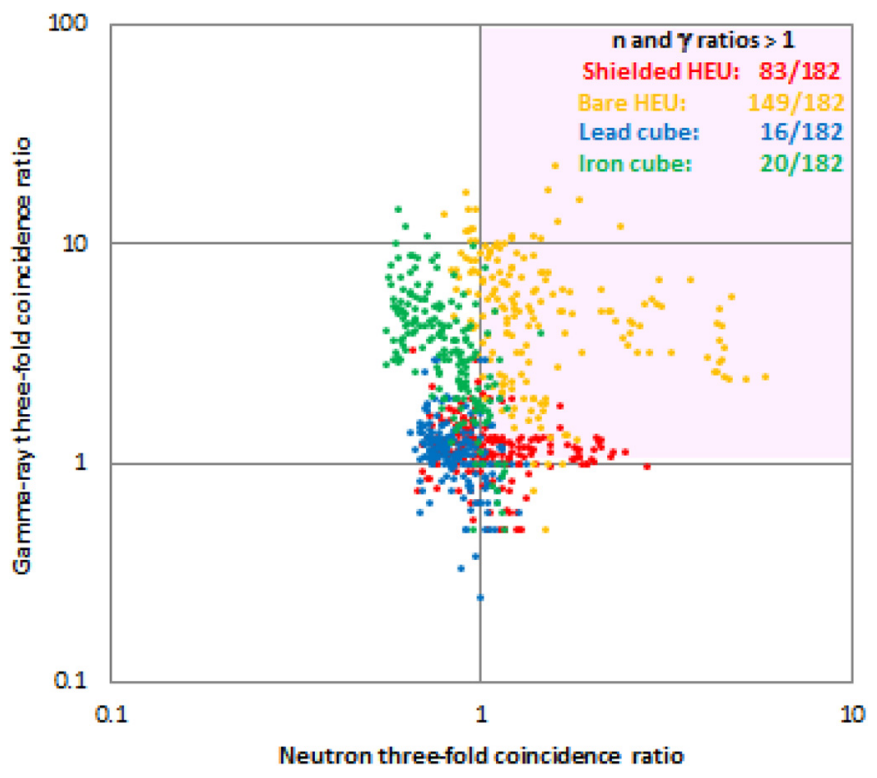

Fig. 47. Correlation between three-fold coincidence ratios (suspicious item/bare wood matrix) calculated in the fission neutron and fission gamma rays windows, respectively. Each point corresponds to one of the 182 studied positions of the suspicious item.

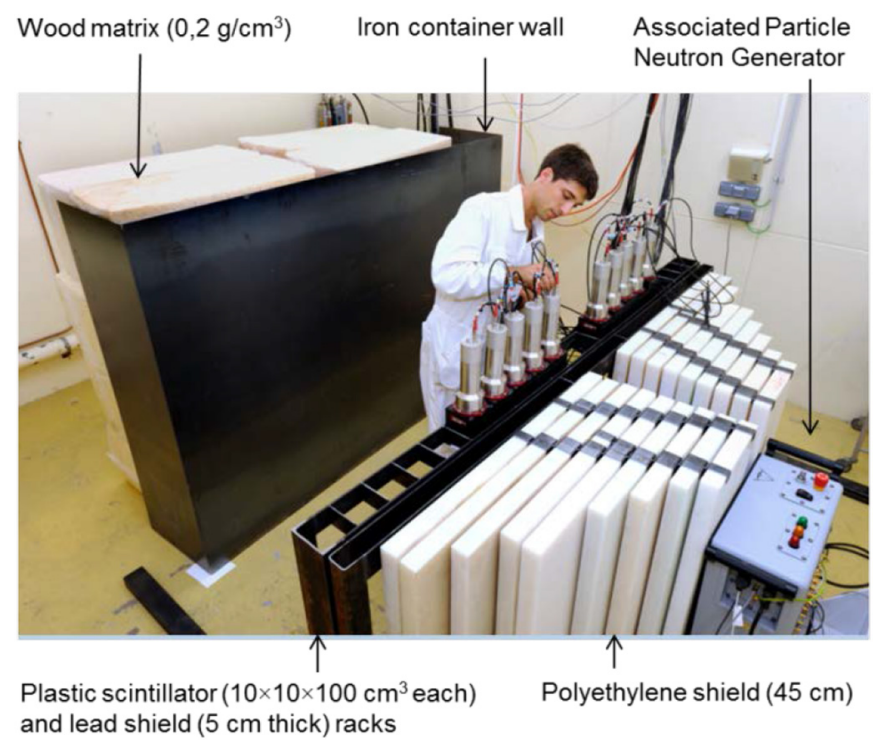

Fig. 48. Experimental setup for the inspection of a container loaded with a $0.2 \mathrm{~g} . \mathrm{cm}^{-3}$ wood matrix. Plastic scintillators are shielded from the generator by $45 \mathrm{~cm}$ polyethylene and $5 \mathrm{~cm}$ lead shields.

Counts (per tagged neutron)
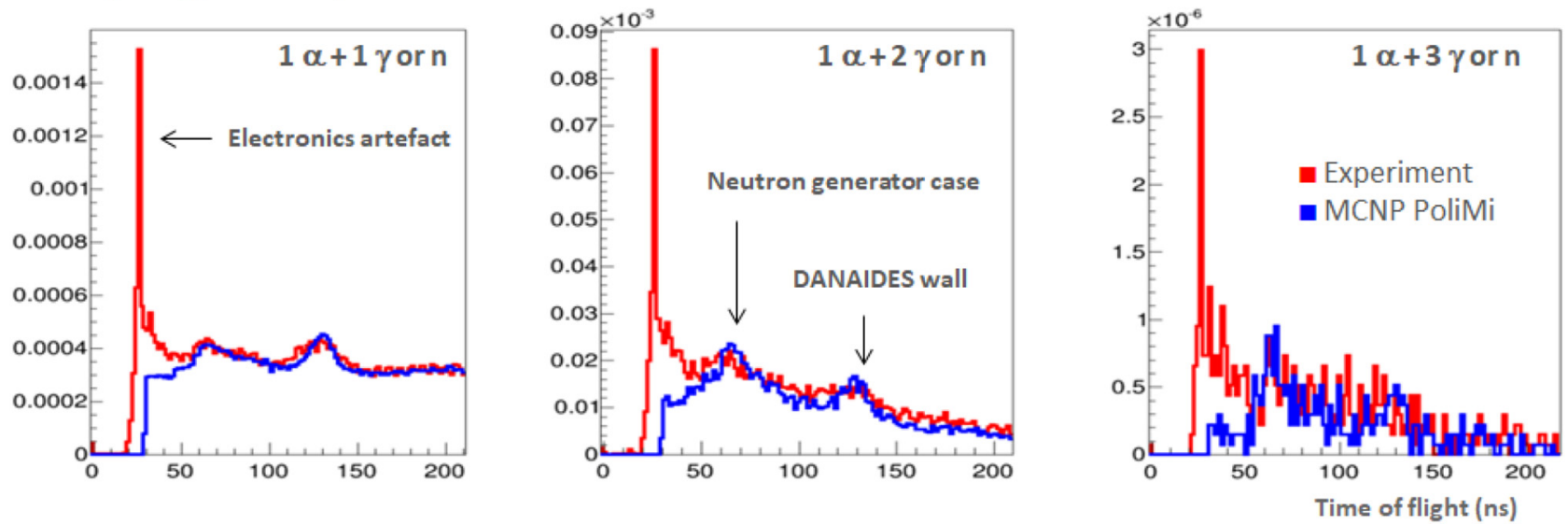

Fig. 49. Time-of-flight background spectra without container, when an alpha particle is detected in coincidence with one (left), two (middle) or three (right) induced gamma rays or neutrons.

Counts (per tagged neutron)

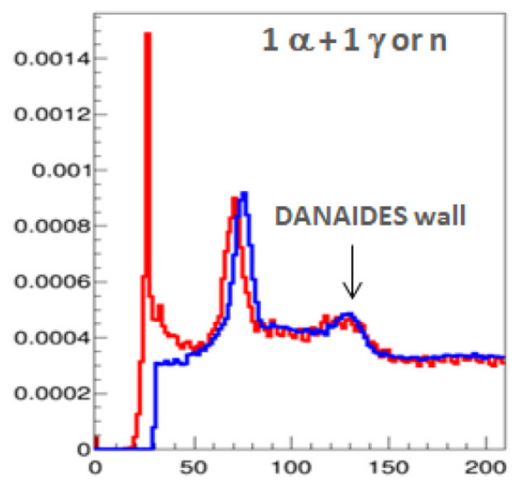

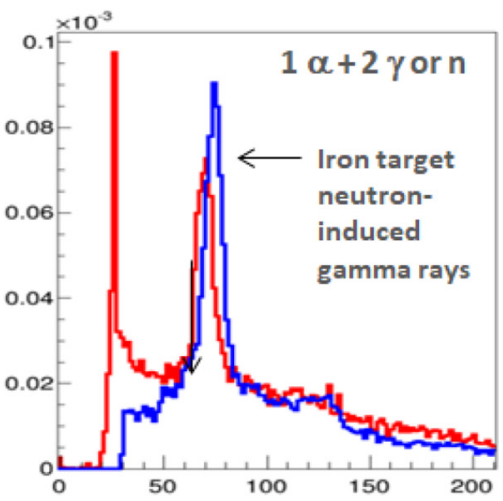

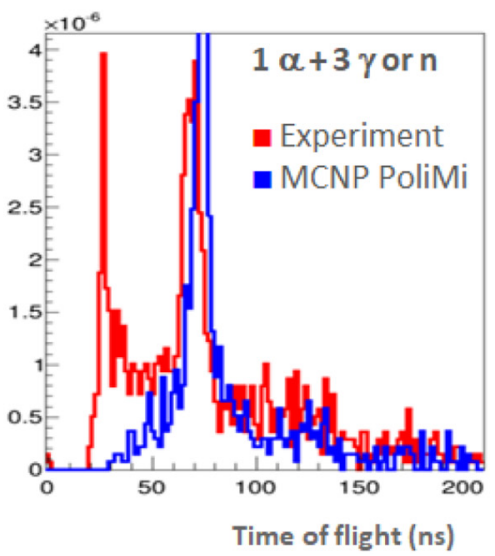

Fig. 50. Time-of-flight spectra with an iron target $(\sim 2.5 \mathrm{~kg})$ inside the empty container, when an alpha particle is detected in coincidence with one, two or three gamma rays or neutrons. 
Counts (per tagged neutron)
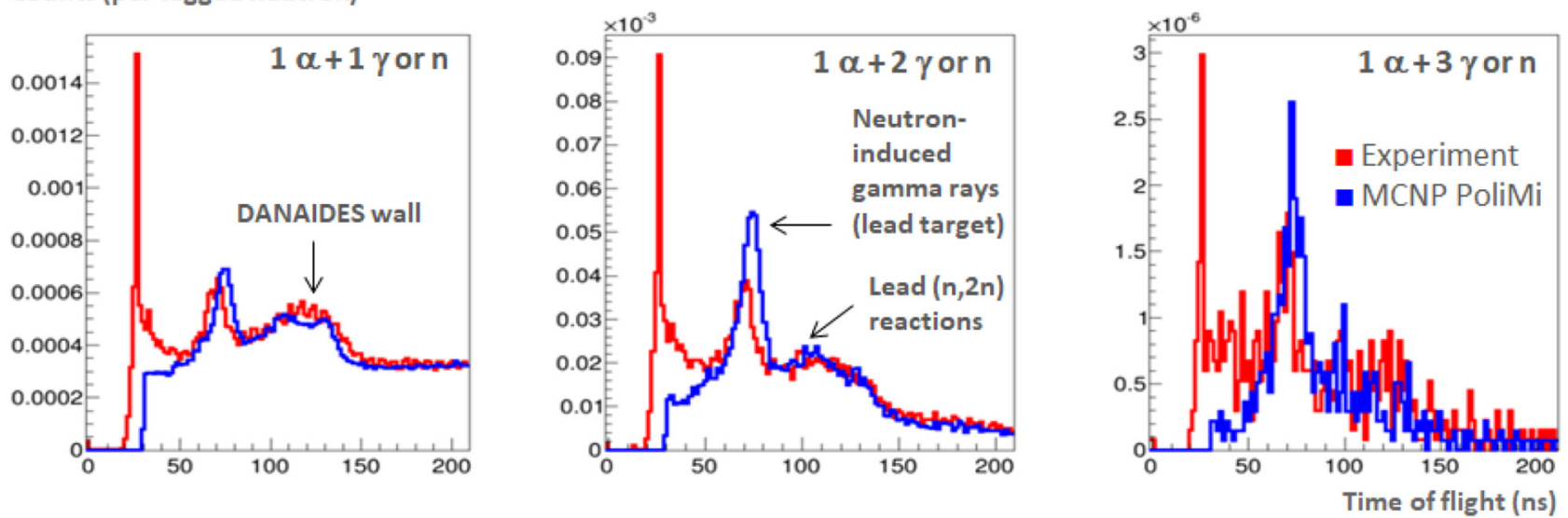

Fig. 51. Time-of-flight spectra with a lead target $(\sim 5 \mathrm{~kg})$ inside the empty container, when an alpha particle is detected in coincidence with one, two or three gamma rays or neutrons.

Counts (per tagged neutron)

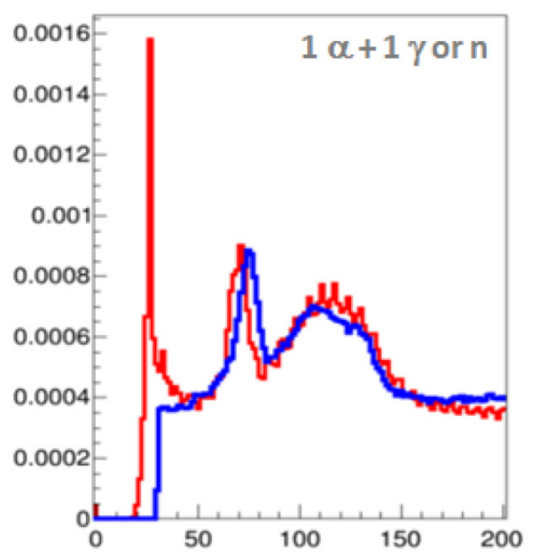

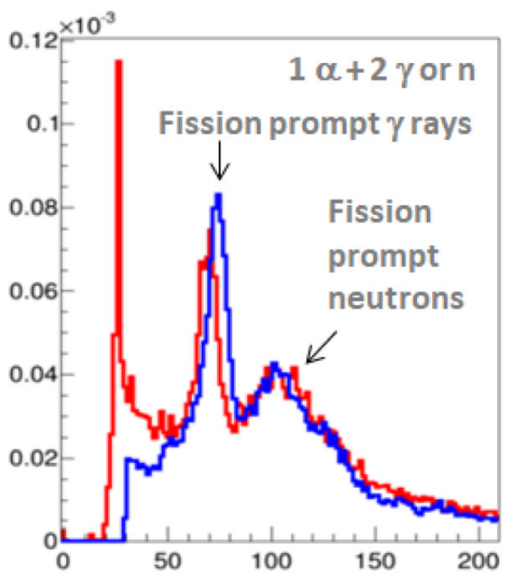

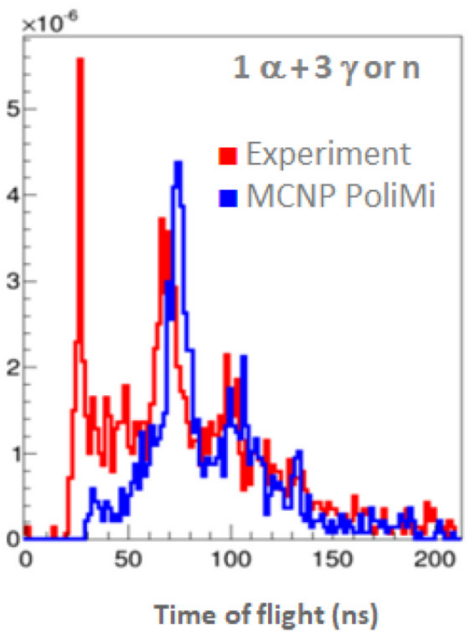

Fig. 52. Time-of-flight spectra with a natural uranium target (a few $\mathrm{kg}$ of metallic uranium with $8 \mathrm{~mm}$ of lead shield around) inside the empty container, when an alpha particle is detected in coincidence with one, two or three gamma rays or neutrons.

\section{Tribute}

The Authors have special thoughts for late Jean-Louis Szabo and Beppe Viesti, who have done so much to give birth to these exciting projects and to put them on the best track. They put a lot of energy, perseverance, intelligence, friendship and heart into this adventure. Today we miss them a lot and we dedicate this paper to their memory.

\section{Author contribution statement}

Bertrand Pérot, Cédric Carasco, Cyrille Eléon, Sébastien Bernard, Alix Sardet, Wassila El Kanawati, Clément Deyglun, Grégory Perret, Guillaume Sannié: numerical simulation, data processing, laboratory and field tests for the development and implementation of Tagged neutron Systems (TNIS).

Vladivoj Valkovic, Davorin Sudac, Jasmina Obhodas: original and founding ideas related to the Associated Particle Technique (APT), implementation of laboratory and field tests.
Sandra Moretto, Giancarlo Nebbia, Cristiano Fontana, Felix Pino: development, integration and data processing of YAP alpha scintillator inside the tagged neutron generator, $\mathrm{NaI}$ and $\mathrm{LaBr}$ gamma scintillator tests, data acquisition and data processing software, laboratory and field tests of the TNIS.

Antonietta Donzella, Aldo Zenoni: Monte Carlo numerical simulation for the design and development of the TNIS.

Alessandro Iovene, Carlo Tintori: data acquisition and processing electronics for the TNIS.

Marek Moszynski, Michal Gierlik: gamma - neutron scintillator design and fine laboratory characterization.

\section{References}

1. S. Ogorodnikov, V. Petrunin, Phys. Rev. Special Top. Accelerators and Beams 5, 104701 (2002)

2. S. Abbasi, M. Mohammadzadeh, M. Zamzamian, A novel dual high-energy X-ray imaging method for materials discrimination, Nucl. Instrum. Methods Phys. Res. Section 
A: Accelerators, Spectrometers, Detectors and Associated Equipment 930, 82-86 (2019)

3. L. Li, R. Li, S. Zhang, T. Zhao, Z. Chen, A dynamic material discrimination algorithm for dual MV energy X-ray digital radiography, Appl. Radiat. Isot. 114, 188-195 (2016)

4. S.P. Osipov, V.A. Udod, Y. Wang, Identification of materials in X-Ray inspections of objects by the dual-energy method, Russ. J. Nondestruct. Test. 53, 568-587 (2017)

5. S.P. Osipov, E. Ju Usachev, S.V. Chakhlov, S.A. Schetinkin, O.S. Osipov, Inspection of bulk cargoes and liquids by the dual energy method, Radiat. Phys. Chem. 177, 109133 (2020)

6. N.G. Cutmore, Y. Liu, J.R. Tickner, Development and commercialization of a fast-neutron/x-ray Cargo Scanner, 2010 IEEE International Conference on Technologies for Homeland Security (HST), https://doi.org/10.1109/ THS.2010.5655030

7. J. Stevenson, T. Gozani, M. Elsalim, C. Condron, C. Brown, Linac based photofission inspection system employing novel detection concepts, Nucl. Instrum. Meth. Phys. Res. A 652, 124-128 (2011)

8. J.C. Overley, M.S. Chmelik, R.J. Rasmussen, R.M.S. Schofield, G.E. Sieger, H.W. Lefevre, Explosives detection via fast neutron transmission spectroscopy, Nucl. Instrum. Meth. Phys. Res. B 251, 470-478 (2006)

9. G. Viesti, L. Cossutta, D. Fabris, M. Lunardon, S. Moretto, G. Nebbia, S. Pesente, F. Pino, L. Sajo-Bohus, Material recognition by using a tagged ${ }^{252} \mathrm{Cf}$ source, Nucl. Instrum. Meth. Phys. Res. Section A: Accelerators, Spectrometers, Detectors and Associated Equipment, Volume 593, Issue 3, 2008, Pages 592-596, ISSN 0168-9002, https://doi.org/ 10.1016/j.nima.2008.05.024

10. David Perticone, Brandon W. Blackburn, Gongyin Chen, Wilbur A. Franklin, Ernest E. Ihloff, Gordon E. Kohse, Richard C. Lanza, Brian McAllister, Vitaliy Ziskin, Fast neutron resonance radiography for elemental imaging, Nucl. Instrum. Meth. Phys. Res. A 922, 71-75 (2019)

11. G. Vourvopoulos, P.C. Womble, Talanta 54, 459 (2001)

12. T. Gozani, Nucl. Instr. Meth. B 213, 460 (2004)

13. D. Strellis, T. Gozani, J. Stevenson, Air cargo inspection using pulsed fast neutron analysis, presented at Proceedings of the International Topical Meeting on Nuclear Research Applications and Utilization of Accelerators, 4-8 May 2009, Vienna, Austria

14. G. Viesti, S. Pesente, G. Nebbia, M. Lunardon, D. Sudac, K. Nađ, S. Blagus, V. Valković, Detection of hidden explosives by using tagged neutron beams: Status and perspectives, Nucl. Instrum. Meth. Phys. Res. B 241, 748-752 (2005)

15. V. Valković, D. Miljanić, P. Tomas, B. Antolković, M. Furić, Neutron-charged particle coincidence measurements from 14.4 MeV neutron induced reactions, Nucl. Instrum. Meth. 76, 29 (1969)

16. G. Nebbia, S. Pesente, M. Lunardon, G. Viesti, P. LeTourneur, F. Heuveline, M. Mangeard, C. Tcheng, Performance of a tagged neutron inspection system (TNIS) based on portable sealed generators, Nucl. Instrum. Meth. Phys. Res. A 533, 475-480 (2004)

17. C. Carasco et al., Photon attenuation and neutron moderation correction factors for the inspection of cargo containers with tagged neutrons, Nucl. Instrum. Meth. Phys. Res. A 582, 638-643 (2007)
18. W. El Kanawati, B. Perot, C. Carasco, C. Eléon, V. Valkovic, D. Sudac, J. Obhodas, Conversion factors from counts to chemical ratios for the EURITRACK tagged neutron inspection system, Nucl. Instrum. Meth. Phys. Res. A 654, 621-629 (2011)

19. J.A. Mullens, P.A. Hausladen, P. Bingham, D.E. Archer, J.T. Mihalczo, Use of imaging for nuclear material control and accountability, ESARDA 29th Annual Meeting, in Symposium on Safeguards and Nuclear Material Management, Aix-en-Provence (France), 22-24 May 2007, pp. 10811090

20. S.G. Belichenko, A.A. Anan'ev, E.P. Bogolyubov, O.V. Bochkarev, E.V. Petrov, A.M. Polishchuk, Y.G. Polkanov et al., Tagged Neutrons from Portable Neutron Generator for Detection of High Explosives and Fissile Materials in Cargo Containers, International Topical Meeting on Nuclear Research Applications and Utilization of Accelerators, Vienna (2009)

21. C. Carasco, C. Deyglun, B. Pérot, S. Normand, G. Sannié, K. Boudergui, G. Corre, V. Kondrasovs, P. Pras, Detection of special nuclear materials with the associate particle technique, CAARI 2012, 22nd International Conference on the Application of Accelerators in Research and Industry, 5-10 August 2012, Fort Worth, Texas, USA

22. R.C. Runkle, D.L. Chichester, S.J. Thompson, Rattling nucleons: New developments in active interrogation of special nuclear material, Nucl. Instrum. Meth. Phys. Res. A 663, 75-95 (2012)

23. C.E. Moss, C.A. Goulding, C.L. Hollas, W.L. Myers, Linear Accelerator-Based Active Interrogation For Detection of Highly Enriched Uranium, Application of Accelerators in Research and Industry: Seventeenth International Conference, 12-16 November 2002, Denton, Texas, AIP Conference Proceedings, Vol. 680, American Institute of Physics, New York, 2003, pp. 900-904

24. E.B. Norman, S.G. Prussin, R.M. Larimer, H. Shugart, E. Browne, A.R. Smith et al., Signatures of fissile materials: high-energy $\gamma$ rays following fission, Nucl. Instrum. Meth. Phys. Res. A 521, 608-610 (2004)

25. M. Agelou, D. Dore, E. Dupont, F. Carrel, M. Gmar, X. Ledoux, B. Perot et al., Detecting special nuclear materials inside cargo containers using photofission, in Nuclear Science Symposium Conference Record (NSS/MIC), IEEE, 2009, pp. 936-939

26. E. Padovani, S.D. Clarke, S.A. Pozzi, Feasibility of prompt correlated counting from photon interrogation of concealed nuclear materials, Nucl. Instrum. Meth. Phys. Res. A 583, 412-420 (2007)

27. P.A. Hausladen, J.T. Mihalczo, B.W. Blackburn, S.M. Watson, J.L. Jones, A.W. Hunt et al., Identifying nuclear material via prompt photo-neutron multiplicity measurements, Application of Accelerators in Research and Industry: Twentieth International Conference, AIP Conference Proceedings, 1099, pp. 670-675 (2009)

28. S. Korbly, Passport Systems, Inc., https://accelconf.web. cern.ch/napac2016/talks/thb2io01_talk.pdf

29. J.M. Hall et al., The nuclear car wash: neutron interrogation of cargo containers to detect hidden SNM, Nucl. Instrum. Meth. Phys. Res. B 261, 337-340 (2007)

30. K.A. Jordan, T. Gozani, Pulsed neutron differential die away analysis for detection of nuclear materials, Nucl. Instrum. Meth. Phys. Res. B 261, 365-368 (2007) 
31. K.A. Jordan, T. Gozani, J. Vujic, Differential die-away analysis system response modelling and detector design, Nucl. Instrum. Meth. Phys. Res. A 589, 436-444 (2008)

32. F.H. Ruddy, R.W. Flammang, J.G. Seidel, Low-background detection of fission neutrons produced by pulsed neutron interrogation, Nucl. Instrum. Meth. Phys. Res. A 598, 518-525 (2009)

33. H. Rennhofer, J.M. Crochemore, E. Roesgen, B. Pedersen, Detection of SNM by delayed gamma rays from induced fission, Nucl. Instrum. Meth. Phys. Res. A 652, 140-142 (2011)

34. J.A. Church, D.R. Slaughter, S. Asztalos, P. Biltoft, M.A. Descalle, J. Hall, T. Luu et al., Signals and interferences in the nuclear car wash, Nucl. Instrum. and Meth. Phys. Res. B 261, 351-355 (2007)

35. J.T. Caldwell et al., Apparatus and method for quantitative assay of generic transuranic wastes from nuclear reactors. United States Patent US 4483816, 1984

36. R.T. Kouzes, J.H. Ely, L.E. Erikson, W.J. Kernan, A.T. Lintereur, E.R. Siciliano, D.L. Stephens, D.C. Stromswold, R.M. Van Ginhoven, M.L. Woodring, Neutron detection alternatives to $3 \mathrm{He}$ for national security applications, Nucl. Instrum. and Meth. Phys. Res. A 623, 1035-1045 (2010)

37. S. Pesente et al., Detection of hidden explosives by using tagged neutron beams with sub-nanosecond time resolution, Nucl. Instrum. Meth. A 531, 657 (2004)

38. B. Perot et al., Proc. SPIE Vol. 6213, Non-Intrusive Inspection Technologies (2006) p. 621305

39. B. Perot et al., Development of the EURITRACK tagged neutron inspection system, Nucl. Instrum. Meth. Phys. Res. B 261, 295-298 (2007)

40. G. Nebbia et al., Development of Tagged Neutron Beams for the EURITRACK (EURopean Illicit TRAfficking Countermeasures Kit) Project, IEEE Nucl. Sci. Symp. Conf. Rec. 1 (2005) 134

41. M. Gierlik, T. Batsch, M. Moszynski, D. Wolski, W. Klamra, B. Pérot, G. Perret, Comparative study of large $\mathrm{NaI}(\mathrm{Tl})$ and BGO scintillators for the EURopean Illicit TRAfficking Countermeasures Kit project, IEEE Trans. Nucl. Sci. 53, 1737 (2006)

42. M. Lunardon et al., Front-end electronics and DAQ for the EURITRACK tagged neutron inspection system, Nucl. Instrum. Meth. Phys. Res. B 261, 391-395 (2007)

43. G. Perret, B. Perot J.-L. Artaud, A. Mariani, EURITRACK tagged neutron inspection system design, J. Phys. Conf. Ser. 41, 375 (2006)

44. A. Donzella, I. Bodini, A. Zenoni, A. Fontana, B. Pérot, S. Bernard, C. Carasco, A. Mariani, D. Sudac, V. Valkovic, Experimental validation of MCNP simulations for the EURITRACK Tagged Neutron Inspection System, Nucl. Instrum. Meth. Phys. Res. B 261, 291-294 (2007)

45. S. Bernard, B. Pérot et al., Development of the EURITRACK Tagged Neutron Inspection System: from simulation to experiment, in: A. Plompen (Ed.), Proceedings of NEMEA-3, 3rd Workshop on Neutron Measurements, Evaluations and Applications, Borovets (Bulgaria), 25-28 October 2006, European Commission report EUR 22794 EN

46. C. Carasco, B. Pérot et al, In-field tests of the EURITRACK tagged neutron inspection system, Nucl. Instrum. Meth. Phys. Res. A 588, 397-405 (2008)

47. B. Pérot, C. Carasco et al., Measurement of $14 \mathrm{MeV}$ neutroninduced prompt gamma-ray spectra from 15 elements found in cargo containers, Appl. Radiat. Isot. 66, 421-434 (2008)
48. W. El Kanawati, C. Carasco, B. Pérot, A. Mariani, A-C. Raoux, V. Valkovic, D. Sudac, J. Obhodas, M. Baricevic, Gamma-ray signatures improvement of the EURITRACK tagged neutron inspection system database, IEEE Trans. Nucl. Sci. 57, 2879-2885 (2010)

49. W. El Kanawati, B. Perot, C. Carasco, C. Eleon, V. Valkovic, D. Sudac, J. Obhodas, G. Sannie, Acquisition of prompt gamma-ray spectra induced by $14 \mathrm{MeV}$ neutrons and comparison with Monte Carlo simulations, Appl. Radiat. Isot. 69, 732-743 (2011)

50. J.F. Briesmeister (Editor), MCNP - A General Monte Carlo N Particle Transport Code (Version 4C), LA-13079-M, Los Alamos National Laboratory, March 2000

51. D.B. Pelowitz, MCNPX ${ }^{\mathrm{TM}}$ USER'S MANUAL Version 2.5.0, LA-CP-05-0369, Los Alamos, USA, 2005

52. B. Perot, W. El Kanawati, C. Carasco, C. Eleon, V. Valkovic, D. Sudac, J. Obhodas, G. Sannie, Quantitative comparison between experimental and simulated gamma-ray spectra induced by $14 \mathrm{MeV}$ tagged neutrons, Appl. Radiat. Isot. 70, 1186-1192 (2012)

53. A.V. Evsenin, I.Y. Gorshkov et al., Detection of explosives and other illicit materials by nanosecond neutron analysis, International Topical Meeting on Nuclear Research Applications and Utilization of Accelerators, 4-8 May 2009, Vienna

54. S.P. Simakov, A. Pavlik, H. Vonach, S. Hlavac, Status of experimental and evaluated discrete gamma-ray production at $\mathrm{En}=14.5 \mathrm{MeV}$, IAEA Nuclear Data Section, INDC(CCP)413, September 1998. https://inis.iaea.org/collection/ NCLCollectionStore/ Public/29/062/29062821.pdf?r $=1$

55. J. Bendahan, J. Clayton, K. Fankhauser, T. Gozani, J. Krivicich, R. Loveman, E. Pentaleri, P. Ryge, P. Sawa, J. Stevenson, Nucl. Instrum. Meth. B 99, 505 (1995)

56. C. Carasco, MCNP Output Data Analysis with ROOT (MODAR), Comp. Phys. Commun. 181, 1161-1166 (2010)

57. J. Obhodas, D. Sudac, V. Valkovic, M. Baricevic, A. Franulovic, B. Perot, C. Carasco, A. Mariani, A.-C. Raoux, W. El Kanawati, Analysis of containerized cargo in the ship container terminal, Nucl. Instrum. Meth. Phys. Res. A 619, 460-466 (2010)

58. M.-A. Descalle, D. Manatt, D. Slaughter, Analysis of Manifests for Containerized Commodities Imported through US Ports, Nucl. Sci. Symp. Conf. Rec. IEEE, 2006, pp. 275-280

59. B. Pérot, C. Carasco, V. Valkovic, D. Sudac, A. Franulovic, Detection of illicit drugs with the EURITRACK system, AIP Conf. Proc. 1099, 565-569 (2009)

60. A. Sardet, B. Pérot, C. Carasco et al., Design of the rapidly relocatable tagged neutron inspection system of the C-BORD project, 2016 IEEE Nuclear Science Symposium, Medical Imaging Conference and Room-Temperature Semiconductor Detector Workshop (NSS/MIC/RTSD)

61. C.L. Fontana, M. Lunardon, F.E. Pino et al., A distributed data acquisition system for signal digitizers with on-line analysis capabilities, 2017 IEEE Nuclear Science Symposium and Medical Imaging Conference (NSS/MIC)

62. C.L. Fontana, A. Carnera, M. Lunardon et al., A distributed data acquisition system for nuclear detectors, Int. J. Mod. Phys. Conf. Ser. 48, 1860118 (2018)

63. C.L. Fontana, M. Lunardon F. Pino, et al., Resource sharing in nuclear physics laboratory classes: a distributed data acquisition system for experiments with shared resources and data management, AIP Conf. Proc. 2160, 050024 (2019), https://doi.org/10.1063/1.5127716 
64. F. Pino, C.L. Fontana, G. Nebbia, S. Moretto et al., Detection module of the C-BORD Rapidly Relocatable Tagged Neutron Inspection System (RRTNIS), Nucl. Instrum. Meth. Phys. Res. A 986, 164743 (2021)

65. A. Sardet, B. Pérot, C. Carasco et al., Performances of C-BORD's tagged neutron inspection system for explosives and illicit drugs detection in cargo containers, IEEE Trans. Nucl. Sci. (2021), http://dx.doi.org/10.1109/TNS.2021.3050002

66. C.L. Fontana, F. Pino, G. Nebbia, S. Moretto et al., Nonintrusive inspection of cargo containers using the C-BORD Rapidly Relocatable Tagged Neutron Inspection System, submitted to Nucl. Instrum. Meth. Phys. Res. A

67. A.G. Sébert, J.-P. Poli, Material classification from imprecise chemical composition: probabilistic vs possibilistic approach, 2018 IEEE International Conference on Fuzzy Systems, 8-13 July 2018, Rio de Janeiro, Brazil

68. C. Carasco, B. Pérot, A. Sardet, Measuring hydrogen with fast neutrons: application to organic materials identification in cargo containers, Nucl. Instrum. Meth. Phys. Res. A 951, $163030(2020)$

69. J. Medalia, Nuclear Terrorism: A Brief Review of Threats and Responses, Updated February 10, 2005, http://ftp.fas. org/sgp/crs/nuke/RL32595.pdf

70. C.D. Ferguson, W.C. Potter, Improvised Nuclear Devices and Nuclear Terrorism, 2006 No2, http://www.wmdcommis sion.org/files/No2.pdf

71. R. Severe William, I. Balatsky Galya, L. Eaton Stacey, Illicit Trafficking of Radioactive and Nuclear Materials. Nuclear Safeguards, Security, and Nonproliferation: Achieving Security with Technology and Policy. Burlington, MA: Butterworth-Heinemann, ch. 22, (2008)

72. IAEA Nuclear Security Series No. 6, Combating illicit trafficking in nuclear and other radioactive material: reference manual, International Atomic Energy Agency, Vienna, 2007

73. J.E. Doyle (Ed.), in Nuclear safeguards, security, and nonproliferation, 2nd Edition, (Butterworth-Heinemann, 2019), pp. 357-387
74. J.T. Mihalczo, Radiation Detection for Active Interrogation of HEU. United States: N. p., 2004. Web. doi: 10.2172/885764

75. A. Oberstedt, R. Billnert, A. Gatera, A. Göök, S. Oberstedt, Prompt gamma rays from ${ }^{252} \mathrm{Cf}(\mathrm{sf})$ and their angular distributions, EPJ Web Conf. 169, 00014 (2018)

76. S.A. Pozzi, E. Padovani, M. Marseguerra, MCNP-PoliMi: a Monte-Carlo code for correlation measurements, NIM A 513, 550 (2003). RSICC Code Package CCC-718/MCNPPOLIMI v1.0

77. R. Brun, F. Rademakers, ROOT - An object oriented data analysis framework, Nucl. Instrum. Meth. A 389, 81-86 (1997)

78. C. Deyglun, C. Carasco, B. Pérot, Monte Carlo parametric studies of neutron interrogation with the Associated Particle Technique for cargo container inspections, SNA-MC 2013, 2nd Joint International Conference on Supercomputing in Nuclear Application (SNA) and Monte Carlo (MC), 27-31 October 2013, Paris

79. S.A. Pozzi, Recent Developments in the MCNP-PoliMi Post processing Code, ORNL/TM-2004/299, Oak Ridge National Laboratory, Oak Ridge, Tennessee (2004)

80. B. Perot, C. Carasco, Method for detecting nuclear material by means of neutron interrogation, and related detection system, WIPO Patent Application WO/2012/095357, https://patent scope.wipo.int/search/en/detail.jsf?docId=WO2012095357

81. C. Deyglun, C. Carasco, B. Pérot, S. Normand, G. Sannié, K. Boudergui, G. Corre, V. Konzdrasovs, P. Pras, Passive and active correlation techniques for the detection of nuclear materials, IEEE Trans. Nucl. Sci. 61, 2228-2234 (2014)

82. R. Beyer et al., Inelastic scattering of fast neutrons from excited states in 56Fe, Nucl. Phys. A 927, 41-52 (2014)

83. R. De Stefano, B. Pérot, C. Carasco, E. Simon, M. Ramdhane, V. Bottau, J. Loridon, C. Eleon, Pulsed neutron interrogation with PVT plastic scintillators to detect nuclear materials, Nucl. Instrum. Meth. Phys. Res. A 976, 164276 (2020)

84. B. Pérot, C. Carasco, C. Deyglun, G. Sannié, J. Gameiro, G. Corre, K. Boudergui, V. Konzdrasovs, Detection Of Special Nuclear Materials with Tagged Neutrons, Nuclear Science Symposium, October 29-November 4, 2016, Strasbourg, France

Cite this article as: Bertrand Pérot, Cédric Carasco, Cyrille Eléon, Sébastien Bernard, Alix Sardet, Wassila El Kanawati, Clément Deyglun, Grégory Perret, Guillaume Sannié, Vladivoj Valkovic, Davorin Sudac, Jasmina Obhodas, Sandra Moretto, Giancarlo Nebbia, Cristiano Fontana, Felix Pino, Antonietta Donzella, Aldo Zenoni, Alessandro Iovene, Carlo Tintori, Marek Moszynski, Michal Gierlik, Sea container inspection with tagged neutrons, EPJ Nuclear Sci. Technol. 7, 6 (2021) 